\title{
At risk for depression : the role of stress and serotonergic vulnerability
}

Citation for published version (APA):

Firk, C. (2009). At risk for depression : the role of stress and serotonergic vulnerability. [Doctoral Thesis, Maastricht University]. Datawyse / Universitaire Pers Maastricht. https://doi.org/10.26481/dis.20090312cf

Document status and date:

Published: 01/01/2009

DOI:

10.26481/dis.20090312cf

Document Version:

Publisher's PDF, also known as Version of record

\section{Please check the document version of this publication:}

- A submitted manuscript is the version of the article upon submission and before peer-review. There can be important differences between the submitted version and the official published version of record.

People interested in the research are advised to contact the author for the final version of the publication, or visit the DOI to the publisher's website.

- The final author version and the galley proof are versions of the publication after peer review.

- The final published version features the final layout of the paper including the volume, issue and page numbers.

Link to publication

\footnotetext{
General rights rights.

- You may freely distribute the URL identifying the publication in the public portal. please follow below link for the End User Agreement:

www.umlib.nl/taverne-license

Take down policy

If you believe that this document breaches copyright please contact us at:

repository@maastrichtuniversity.nl

providing details and we will investigate your claim.
}

Copyright and moral rights for the publications made accessible in the public portal are retained by the authors and/or other copyright owners and it is a condition of accessing publications that users recognise and abide by the legal requirements associated with these

- Users may download and print one copy of any publication from the public portal for the purpose of private study or research.

- You may not further distribute the material or use it for any profit-making activity or commercial gain

If the publication is distributed under the terms of Article $25 \mathrm{fa}$ of the Dutch Copyright Act, indicated by the "Taverne" license above, 
AT RISK FOR DEPRESSION

The role of stress and serotonergic vulnerability 


\section{Colophon}

Graphic design: Christine Firk

Production: Datawyse, Universitaire Pers Maastricht

(C) Christine Firk, Maastricht 2009

ISBN 9789052788098

Publication of this thesis was financially supprted by DSM Food Specialties Delft. 


\section{AT RISK FOR DEPRESSION}

The role of stress and serotonergic vulnerability

\section{PROEFSCHRIFT}

ter verkrijging van de graad van doctor aan de Universiteit Maastricht, op gezag van de Rector Magnificus, Prof. mr. G.P.M.F Mols, volgens het besluit van het College van Decanen, in het openbaar te verdedigen op donderdag 12 maart 2009 om 16 uur

door

Christine Firk

geboren op 21 oktober 1980 te Aken

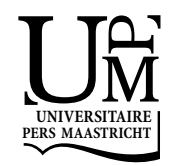




\section{Promotor}

Prof. dr. A. T. M. Jansen

Copromotor

Dr. C.R. Markus

Beoordelingscommissie

Prof. dr. W. Riedel (voorzitter)

Dr. A. Blokland

Prof. dr. A. J. W. van der Does (Universiteit Leiden)

Dr. F. Peeters

Prof. dr. H. M. van Praag 


\section{CONTENTS}

Chapter 1 General Introduction

Chapter 2 Effects of acute tryptophan depletion on affective processing in first-degree relatives of depressive patients and controls after exposure to uncontrollable stress

Chapter 3 Effects of 5-HTTLPR genotype and acute tryptophan depletion on affective processing following acute stress exposure

Chapter 4 Differential effects of 5-HTTLPR genotypes on mood, memory and attention bias following acute tryptophan depletion and stress exposure

Chapter 5 Effect of different tryptophan sources on amino acids availability to the brain and mood in healthy volunteers

Chapter 6 Mood and cortisol responses following tryptophan augmentation and acute stress in healthy subjects with high and low cognitive reactivity to depression

Chapter 7 General Discussion

Summary

Samenvatting

References

Dankwoord

CV 



\section{CHAPTER 1}

General Introduction

Based on:

Firk, C., Markus, C.R. (2007). Review: Serotonin by stress interaction: a susceptibility factor for the development of depression? Journal of Psychopharmacology 21, 538-544. 


\section{INTRODUCTION}

Depression is one of the most common diseases in the world (Akiskal, 2005). Epidemiological studies suggest a life-time prevalence of Major Depressive Disorder that ranges from 5\% to 17\% (Rihmer \& Angst, 2005). Additionally, its incidence is increasing. Major depression is characterized by depressed mood, loss of interest in nearly all things, and an inability to experience pleasure and is often accompanied by loss of appetite, insomnia, and suicidal ideation (APA, 1994). Thus, depression dramatically damages the quality of life. However, in spite of intensive research during the past decades, critical risk factors involved in the onset or development of depression have not been defined.

Genetic/family predisposition or 'susceptibility' to depression may be a potential risk factor in the development of depression. Family studies have shown that depression is two- to threefold more common in firstdegree relatives compared to the general population (Sullivan et al., 2000; Kelsoe, 2005) and is also associated with early onset and higher levels of morbidity (Lieb et al., 2002). Twin studies, a powerful tool used to differentiate genetic and environmental influences, reveal a higher concordance rate for monozygotic twins than for dizygotic twins (Kendler \& Gardner, 2001), which further corroborates the utility of genetic predisposition as a risk factor in the development of depression. Although the neurobiological equivalent of this genetic predisposition remains unclear, it seems as though the brain serotonin (5-Hydroxytryptamine, 5HT) neurotransmitter system serves an important mediating role (Owens \& Nemeroff, 1994). Alterations in brain serotonergic function is thought to be involved in the onset and course of depression (Maes \& Meltzer, 1995).

Evidence suggests that individuals with a serotonergic vulnerability, which has been defined as vulnerability or sensitivity of the brain serotonergic system to alterations or disruptions, have an increased likelihood of developing disorders that are related to the serotonergic system, such as depression (Jans et al., 2007). Serotonergic vulnerability has been postulated for individuals with a family history of depression $(\mathrm{FH}+)$ (Benkelfat et al., 1994; Klaassen et al., 1999) and for individuals with allelic variation in genes that encode proteins that are critical in determining the overall level of serotonergic neurotransmission (e.g. Neumeister et al., 2002). A major problem, however, is that the role of brain 5-HT in 
depression remains rather complex and it is unlikely that this neurotransmitter is entirely responsible for the pathogenesis of depression (Maes \& Meltzer, 1995). Even though serotonergic vulnerability to depression may constitute a likely risk factor in the development of depression, it does not seem to be the sole contributor. Based on previous data, stress has been suggested as an important additional factor. Depression can be precipitated in susceptible individuals by a series of stressful life events. Indeed, individuals who frequently experience severe stressful life events (for instance, loss, humiliation, or defeat) are more likely to develop major depression relative to individuals who do not experience such major stressful events (Brown et al., 1987; Heim \& Nemeroff, 2001). However, even though major stress has frequently been found to precede the onset of depressive symptoms, this observation does not allow for the drawing of causal conclusions. It is still vital to know when and how stress may cause depressive symptoms and how biological mechanisms may interfere or be involved. Therefore, evidence detailing whether stress generates or promotes biological (brain) dysfunctions that are typically found in depression is required (Van Praag, 2004). This is, indeed, suggested by considerable evidence for dysfunctional stress hormone regulation in depressive patients and for complex inter-relationships between the serotonergic brain system and neuroendocrine mechanisms involved in stress perception and stress adaptation (Arborelius et al., 1999; Holsboer, 2000; Van Praag, 2004; Markus, 2008). In addition, challenges to either the brain serotonergic system or neuroendocrine stress mechanisms involved in stress perception and adaptation may (further) interrupt the complex neurophysiological balance and promote the development of depression (Porter et al., 2004; Markus, 2008). Based on these new insights, it is assumed that serotonergic vulnerability, particularly under major or prolonged stress exposure, may constitute a risk factor for depression. This constitutes the main research question of the thesis. In the following part of this chapter, the rationale for this assumption and the research question will be provided. First, the role of 5-HT in depression will be discussed; next, we will focus on stress as an additional risk factor in the development of depression. Then, the hypothesized interaction between stress, $5-\mathrm{HT}$, and depression will be discussed; evidence for this hypothesis will be given and questions will be raised. In the final part, the aim of the dissertation will be summarized and the methodology of the current studies will be briefly explained. 


\section{SEROTONIN AND DEPRESSION}

The monoamine neurotransmitter $5-\mathrm{HT}$ is involved in most physiologic or behavioral functions (e.g., the regulation of emotion, mood, sleep, appetite, and aggression). Therefore, most psychopharmacological treatments of psychiatric disorders are oriented towards the brain serotonergic system. The important role of the serotonergic system in so many psychological and physiological processes is understandable when looking at the anatomy of the system. The cell bodies of the serotonergic neurons are clustered in the brainstem raphe nuclei and project widely throughout the cortex and limbic system (Aghajanian \& SandersBush, 2002). Since 5-HT cannot pass the blood-brain barrier, its synthesis within the brain depends on the influx of its plasma amino acid precursor tryptophan (TRP). In the brain, two steps are involved in 5-HT synthesis. First, the enzyme tryptophan hydroxylase converts TRP to 5hydroxytryptophan (5-HTP). Second, 5-HTP is acted upon by aromatic amino acid decarboxylase (AADC) to form 5-HT. 5-HT action is terminated by reuptake via the $5-\mathrm{HT}$ transporter (5-HTT) and subsequent degradation by monoamine oxidase (MAO) to 5-hydroxyindoleacetic acid (5HIAA) (e.g. Fernstrom, 1990) (Figure 1).

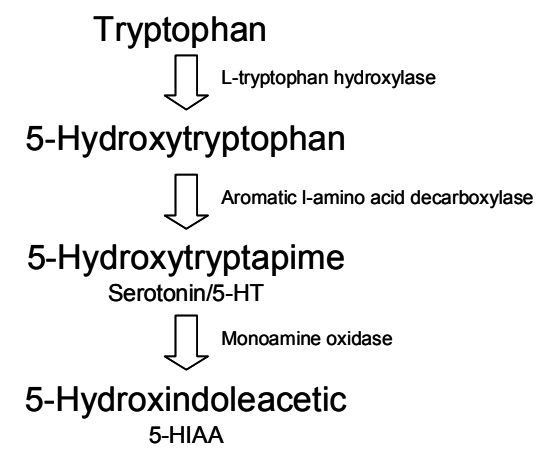

Figure 1. Serotonin synthesis. Serotonin (5-HT) is synthesized from tryptophan in two steps by the enzymes tryptophan hydroxylase and aromatic amino acid decarboxylase. Its breakdown is catalyzed by monoamine oxidase.

The exact role of 5-HT in depression is still unclear; however, it is widely accepted that diminished function of the brain serotonergic system plays a key role in the onset and course of depression (Maes \& Meltzer, 1995; Neumeister et al., 2004c). Evidence supporting reduced brain 5 -HT function in depression comes from studies showing lower plasma 
availability of the 5-HT precursor, TRP, for uptake into the brain, reduced cerebrospinal fluid (CSF) concentration of the 5-HT metabolite 5-HIAA, and decreased platelet 5-HT uptake in depression (Maes \& Meltzer, 1995; Neumeister et al., 2004c). Together, these studies suggest diminished brain 5-HT uptake and metabolism in depressive patients. In addition, disturbances of 5-HT receptor function have been described. The disturbances primarily involve altered postsynaptic $5-\mathrm{HT}_{2 \mathrm{~A}}$ receptor and pre-synaptic $5-\mathrm{HT}_{1 \mathrm{~A}}$ receptor functioning, which results in the dysregulation of 5-HT neurotransmission (Stahl, 1998). The state of the 5- $\mathrm{HT}_{2}$ receptor system remains rather unclear, with some studies reporting upregulation (e.g. Biegon et al., 1990; Pandey et al., 1990) and others reporting downregulation (Audenaert et al., 2001) of $5-\mathrm{HT}_{2 \mathrm{~A}}$ receptors in depression. This variability may indicate different levels of $5-\mathrm{HT}_{2}$ dysfunction in varying areas of the brain. Depression is more consistently associated with presynaptic 5- $\mathrm{HT}_{1 \mathrm{~A}}$ receptor upregulation and postsynaptic 5$\mathrm{HT}_{1 \mathrm{~A}}$ receptor downregulation (Maes \& Meltzer, 1995; Van Praag et al., 2004). Additionally, direct evidence of $5-\mathrm{HT}_{1 \mathrm{~A}}$ receptor pathology in depression comes from imaging studies using Positron Emission Tomography (PET) that show reduced 5- $\mathrm{HT}_{1 \mathrm{~A}}$ receptor binding in depressed patients (Drevets et al., 1999; Sargent et al., 2000). However, these abnormalities do not seem to be specific for depression as they have also been found in patients with panic disorder with and without depression (Neumeister et al., 2004a). Moreover, involvement of other 5-HT receptor subtypes has not been clearly investigated due to a lack of specific receptor ligands.

In addition to 5-HT involvement in depression, allelic variations in the gene that encodes the 5-HT transporter protein (5-HTT) are currently being explored as a genetic risk factor for depression (Mann et al., 2000; Neumeister et al., 2002; Uher \& McGuffin, 2008). 5-HTT plays a crucial role in serotonergic neurotransmission by facilitating reuptake of 5-HT into the presynaptic neuron (Lesch et al., 1996). Fewer 5-HTT sites have been reported in functional imaging studies of depressive patients (Malison et al., 1998) as well as in postmortem studies (Mann et al., 2000), indicating less 5-HTT binding in depressive patients compared to healthy controls. The 5-HTT gene maps to chromosome 17q11.1-q12 (Lesch et al., 1994) and evidence revealed a 5-HTT gene-linked polymorphic region (5-HTTLPR) with two common alleles or variants: the short form (s) and the long form (I) (Heils et al., 1996). The $s$ allele of this 
variant is less active, resulting in reduced transcriptional efficiency of the 5-HTT gene, decreased 5-HTT expression, and reduced 5-HT uptake relative to the I allele (Heils et al., 1996; Greenberg et al., 1999). The functional effect on 5-HT availability suggested an association between the s allele and depression-related phenotypes. Some data, indeed, reveal associations between the $s$ allele and negative emotionality in adults (Lesch et al., 1996) and infants (Auerbach et al., 1999). Furthermore, individuals homozygous for the $s$ allele are more likely to have multiple first-degree relatives with a history of depression (Joiner et al., 2003) and depressed patients (Collier et al., 1996) as well as suicide victims (Bondy et al., 2000) were more likely to carry an s allele. Moreover, it has been found that 3,4-methylenedioxymethamphetamine (MDMA, or Ecstasy) users carrying the $s$ allele show abnormal emotional processing and higher depression scores compared to ecstasy users homozygous for the I allele and control subjects carrying the s allele. MDMA binds to the 5$\mathrm{HTT}$, preventing uptake and stimulating the release of $5-\mathrm{HT}$. These events cause long-term changes to the 5-HT system (Roiser et al., 2005). However, findings concerning an association between the 5-HTT polymorphism and depression have been inconsistent. Most studies revealed an association between the s allele and depression (Collier et al., 1996; Neumeister et al., 2002; Joiner et al., 2003; Gonda et al., 2005; Gonda et al., 2006) but not all demonstrated this finding (Willis-Owen et al., 2005). Inconsistent results detailing the association between 5-HTTLPR and depression may be due to ethnicity or gender effects on 5-HT neurotransmission (Williams et al., 2003) or may be due to recently identified allelic variants within the I allele of the 5-HTTLPR (Hu et al., 2006; Zalsman et al., 2006). The 5-HTTLPR polymorphism is, in fact, tri-allelic due to functional variants within the $I$ allele, designated as $I_{G}$ and $I_{A}$. The $I_{G}$ allele has a low rate of 5-HTT expression comparable to the $s$ allele and is relatively common in Caucasian and African American populations ( $\mathrm{Hu}$ et al., 2006; Zalsman et al., 2006). There is also a paradox in the observation that low 5-HT uptake resulting from the 5-HTTLPR s allele may increase the likelihood of developing depression, whereas low 5-HT reuptake caused by selective serotonin re-uptake inhibitors (SSRIs) is a wellaccepted treatment for depression. The latter issue, however, may arise from the fact that the reduced uptake of 5-HT caused by the 5-HTTLPR s allele occurs throughout development and post-natal life and, consequently, may cause permanent changes in the developing brain, whereas 
SSRIs reduce 5-HT uptake only when administered in adulthood, when the brain is fully developed and less subjected to plasticity and structural-functional changes (Wurtman, 2005). Nevertheless, this paradox remains unresolved.

It seems as though a predisposition to altered $5-\mathrm{HT}$ functioning in certain populations of healthy subjects may constitute a significant risk factor in the development of mood disorders such as depression. Such a 'serotonergic vulnerability for depression' may particularly be involved in first-degree family members of depressed patients and in individuals carrying the s allele 5-HTTLPR, who appear to have an increased risk for the development of major depression (e.g. Reich et al., 1987; Collier et al., 1996; Sullivan et al., 2000). Support for diminished 5-HT functioning in these populations is derived from studies using the acute tryptophan depletion (ATD) strategy (Young et al., 1985). Brain 5-HT synthesis and activity is lowered by depletion of the amount of plasma TRP relative to the sum of the other large neutral amino acids (LNAAs, i.e., valine, leucine, isoleucine, phenylalanine, and tyrosine) with which TRP competes for uptake into the brain (Fernstrom \& Wurtman, 1972). This is accomplished through administration of a balanced TRP-free amino acid mixture, which contains all essential amino acids except for TRP. This reduces the amount of plasma TRP as compared to the LNAAs (TRP:LNAA ratio) by raising incorporation of TRP into protein synthesis (lowering its plasma availability) and, thus, increasing competition of the LNAAs with which TRP competes for uptake into the brain (e.g. Gessa et al., 1974; Moja et al., 1991; Maes \& Meltzer, 1995). Evidence for reduced 5-HT neurotransmission after ATD comes from a broad range of different animal studies measuring 5-HT directly in the brain (e.g. Gessa et al., 1974; Moja et al., 1989) as well as from human PET imaging studies (Nishizawa et al., 1997) and human studies measuring CSF 5-HIAA concentrations (Carpenter et al., 1998; Williams et al., 1999).

Healthy subjects with a positive family history of depression showed significantly greater depressed mood after ATD than healthy controls without a family history of depression (Benkelfat et al., 1994; Klaassen et al., 1999). This was also found for 5-HTTLPR s allele carriers compared to I allele carriers (e.g. Neumeister et al., 2002; Roiser et al., 2007; Walderhaug et al., 2007). Further, ATD is found to cause depressive relapse in depressive patients treated with (and responding to) monoamine oxidase inhibitors (MAOls) or SSRIs (Delgado et al., 1990; Delgado et al., 
1994; Delgado et al., 1999; for a review on tryptophan depletion in psychiatric populations see Bell et al., 2001; Van der Does, 2001), whereas in healthy subjects, mood lowering effects of ATD are not found or appear to be rather modest (e.g. Benkelfat et al., 1994; Bhatti et al., 1998; Klaassen et al., 1999). Recently, it has been shown that depressive relapse to ATD can be predicted by cognitive reactivity to depression (Booij \& Van der Does, 2007), suggesting that cognitive reactivity, a psychological vulnerability marker of depression, is also associated with serotonergic vulnerability.

The mood-lowering findings of ATD in individuals with an increased risk of depression strongly support the assumption that serotonergic vulnerability may constitute an important risk factor for the development of depression. In particular, the increased risk of depression in subjects with a positive family history of depression and in $s$ allele carriers suggests that a strong genetic component promotes brain (5-HT) vulnerability. Despite the fact that this genetic vulnerability may promote the development of depression, many individuals with a positive family history of depression and/or with an s allele 5-HTTLPR do not develop depression (Sullivan et al., 2000; Lotrich \& Pollock, 2004). Thus, the role of 5-HT vulnerability in depression remains rather complex and there does not seem to be a direct relationship between $5-\mathrm{HT}$ dysfunction and symptoms of depression. Finding a solution for this problem constitutes a major challenge in biological psychiatry today. Therefore, gene-environment interactions have been suggested in the etiology of depression. Because depression is often preceded by stress, researchers have hypothesized that a genetic vulnerability to depression, related to the serotonergic system, might only be expressed if an individual is exposed to stress (Van Praag, 2004). Hence, bi-directional interactions are proposed between the stress system and the serotonergic system. Ultimately, these interactions may induce or increase serotonergic dysfunction and promote the development of a depressive disorder.

\section{STRESS: A RISK FACTOR FOR DEPRESSION?}

Stress, although difficult to define, generally refers to a situation during which individuals are activated to prepare adaptive responses to interruptive events that challenge their homeostatic balance or selfcontrol. In order to re-establish homeostasis, a stress-inducing event 
(stressor) should be followed by a physiological stress response (Akil \& Morano, 1995). One of the earliest contributions to stress (response) research comes from Walter Cannon's (1932) description of the fight-orflight response of the sympathetic nervous system (SNS) in response to threat. Cannon found that SNS activation prepares the body for adaptation; either to fight off an attacker or to flee from the danger. Hans Selye (1936; 1976) further popularized the stress concept by proposing that all individuals physiologically respond to all types of threatening situations in the same non-specific manner, which he called the general adaptation syndrome (GAS). In contrast to Cannon, Selye mainly focused on the activation of the hypothalamic-pituitary-adrenal (HPA) axis, which he first described as an adaptive stress response but later discovered that these processes, when excessive, can also damage the body (e.g. Selye, 1976).

In response to stress, SNS levels of noradrenaline increase and activate the sympathetic adrenal medullary system (SAM) to release adrenaline into the bloodstream. The activation of the SNS and the SAM form the body's immediate stress response, increasing heart rate, blood pressure, and blood glucose levels to prepare the organism to survive by either running or fighting (e.g. Clow, 2001). In addition, with some seconds delay, the HPA axis is activated. The HPA axis constitutes the major stress-adaptation mechanism that, on the one hand, provides extra glucose for the sympathetic stress responses and behavioral action and, on the other hand, suppresses the stress response in order to re-establish a physiological balance (Ursin \& Olff, 1993). The HPA axis consists of three components: the hypothalamus, the anterior pituitary, and the adrenal cortex (Arborelius et al., 1999) (Figure 2).

Upon receiving various limbic inputs indicative of stress, cell bodies at the level of the paraventricular nucleus (PVN) of the hypothalamus are stimulated to enhance the release of corticotropin-releasing hormone $(\mathrm{CRH}) . \mathrm{CRH}$, in turn, is the main modulator for cell bodies in the anterior pituitary gland to secrete adrenocorticotropic hormone (ACTH) and related peptides that originate from the same precursor proopiomelanocortin. ACTH is secreted into the systemic circulation and stimulates the adrenal cortex to release glucocorticoid cortisol. Cortisol, in turn, re-establishes the internal balance of the nervous system and the body by exerting a variety of actions throughout the brain in order to terminate the stress-response, recover from stress, and prepare the organism for stress coping (Sapolsky, 1992; Ursin \& Olff, 1993; Dinan, 
1994). These effects of cortisol are regulated by fast and slow negative feedback mechanisms on several levels of the HPA axis resulting in reduced release of $\mathrm{CRH}$ and $\mathrm{ACTH}$. Hence, cortisol binds to receptors at the level of the hippocampus, hypothalamus, and pituitary to mediate negative feedback to the HPA axis (e.g. Fulford \& Harbuz, 2005). Rapid feedback occurs within minutes, primarily by inhibiting $\mathrm{CRH}$ and ACTH release at the level of the PVN and anterior pituitary (Steckler et al., 1999). Delayed feedback emerges 1-2 hours later via an inhibitory action of cortisol at the level of the hippocampus to prevent continued activation of the HPA axis (Fulford \& Harbuz, 2005).

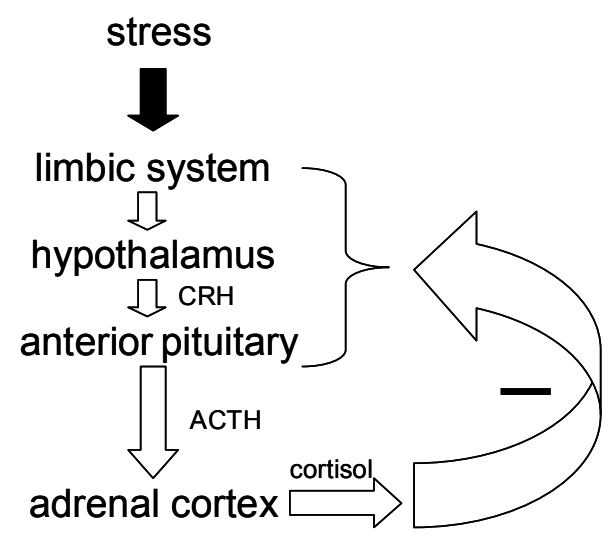

Figure 2. The HPA stress-response. Limbic inputs indicative of stress trigger the hypothalamus to release corticotropin-releasing hormone (CRH). $\mathrm{CRH}$ stimulates the anterior pituitary gland to secrete adrenocorticotropic hormone (ACTH). ACTH stimulates the adrenal cortex to release glucocorticoid cortisol. The HPA axis is regulated by negative feedback of cortisol.

The stress response is essential for adaptation to acute physical or psychological stressors, but may be damaging to the body and brain when chronically activated. This has led to the introduction of two terms: allostasis (i.e., the process of maintaining stability (homeostasis) by the release of stress hormones) and allostatic load or overload (i.e., the wear and tear on the body and brain when stress hormones are chronically activated) (McEwen, 2000). Exposure to chronic stress is thought to negatively affect various brain structures, particularly the hippocampus, by excess circulating glucocorticoids. The hippocampus plays an important role in regulating HPA axis activity; therefore, damage to the hippocampus may cause even higher glucocorticoid levels, which, in turn, in- 
creases hippocampal atrophy and promotes HPA hyperactivity (Sapolsky et al., 1986; Sapolsky, 1996). There is ongoing evidence that the HPA system may be hyperactive in depression (for a detailed review on the role of the HPA system in depression see Steckler et al., 1999; Holsboer, 2000; De Kloet et al., 2005). In the majority of studies, half of depressive patients respond with hyperactivation of the HPA axis (Nestler et al., 2002). Disturbances in the function of the HPA axis result in increased levels of circulating $A C T H$, increased urinary cortisol excretion, and increased levels of CRH in CSF (Holsboer, 2000; Van Praag et al., 2004). Neuroendocrine function tests have been used to further investigate abnormal HPA function. Depressive patients show reduced suppression of ACTH and cortisol after pretreatment with 1 to $2 \mathrm{mg}$ dexamethasone (i.e., the dexamethasone (DEX) suppression test) in comparison to healthy controls (e.g. Carroll, 1982). In depressive patients, the normal degree of cortisol suppression requires higher doses of dexamethasone, suggesting that the cortisol-mediated negative feedback is changed to a higher setpoint (Modell et al., 1997). Additionally, intravenous administration of $\mathrm{CRH}$ leads to an increased ACTH response but normal cortisol response in depressed patients compared to controls (Holsboer et al., 1986). More recently, a combined DEX-CRH test has been used to investigate abnormal HPA function. It was found that dexamethasone pretreated depressed patients show enhanced ACTH and cortisol response to $\mathrm{CRH}$ compared to healthy controls (e.g. Von Bardeleben \& Holsboer, 1989; Rybakowski \& Twardowska, 1999). Moreover, the hormonal response pattern following the combined DEX-CRH test of individuals with a family history of depression was comparable to the enhanced response typically found in depressed patients, suggesting that the vulnerability for HPA axis abnormalities observed in depression may also be (at least partly) genetically determined (Holsboer et al., 1995; Modell et al., 1998).

\section{SEROTONIN, STRESS AND DEPRESSION}

Biological as well as psychological factors are involved in depression and recent data suggests that serotonergic vulnerability and cognitive stress perception may mutually increase the risk of depression. In support of this assumption, interactions between 5-HT and the regulation of neuroendocrine stress mechanisms have been clearly reported. Interest- 
ingly, challenges of either system may reduce the function of the other (Porter et al., 2004).

5-HT plays an important role in regulating HPA axis activity and stress coping and there are strong interrelationships between stress and $5-\mathrm{HT}$ function. In various animal models, it has been shown that $5-\mathrm{HT}$ is important in activating the HPA axis by stimulating $\mathrm{CRH}$ release, triggering ACTH release, and stimulating corticosteroid secretion (Lefebvre et al., 1992; Fuller, 1996). The serotonergic activation of the HPA axis has also been suggested for humans (Dinan, 1996). Furthermore, in animals, it has been found that acute stress leads to a rise in brain 5-HT turnover by increasing TRP availability and stimulating tryptophan hydroxylase (e.g. De Kloet et al., 1982; De Kloet et al., 1983; Davis et al., 1995). The increased release of brain $5-\mathrm{HT}$ is important because it enhances the negative feedback control of cortisol on the HPA axis as a biological mechanism for stress adaptation (Nuller \& Ostroumova, 1980; Van Praag et al., 2004). Conversely, dysfunctional brain 5-HT activity may deteriorate HPA function and reduce stress adaptation in animals (Seckl \& Fink, 1991) and humans (Maes et al., 1991). In animals, continued stress exposure or chronic stress is found to have a negative influence on the 5-HT system and may increase 5-HT sensitivity or vulnerability as a compensatory response (Adell et al., 1988). The existence of a clear mutual relationship between reduced 5-HT function, reduced stress adaptation, and subsequent increased vulnerability to mood deterioration was further suggested by findings that increases in brain $5-\mathrm{HT}$ improve stress coping and, subsequently, lead to reduced depressive mood in healthy stresssusceptible subjects but not in controls (e.g. Markus et al., 1998; Markus et al., 2000a). In addition, the link between 5-HT, stress, and depression has also been investigated in a recent study using ATD (Richell et al., 2005) showing that healthy subjects were more susceptible to the moodlowering effect of uncontrollable stress exposure after ATD as compared to placebo.

Based on these previous findings, serotonergic vulnerability for depression may be accompanied by reduced stress coping mechanisms during exposure to acute stressful situations. As a consequence, the experience of severe stress in these 5-HT-vulnerable subjects may further challenge the 5-HT system which, in turn, may further increase serotonergic vulnerability and, ultimately, increase the risk of developing depression. Support of the assumption that stress, in combination with se- 
rotonergic vulnerability, may increase the risk for depression comes from recent studies showing that 5-HTTLPR can moderate the influence of stress on the onset of depression (Caspi et al., 2003; Kendler et al., 2005). For instance, Caspi et al. (2003) found that individuals carrying one or two s alleles of the 5-HTTLPR were more sensitive to the depressogenic effects of stressful life events than individuals homozygous for the I allele. However, Kendler et al. (2005) found that the interaction between stressful life events and genotype resulted from an increased sensitivity to depressogenic effects for individuals homozygous for the s allele. The gene-environment interaction has also been investigated in maltreated children (Kaufman et al., 2004). Maltreated children with the $\mathrm{s} / \mathrm{s}$ genotype had higher depression scores than control children with the same genotype. Interestingly, this was also influenced by the social support that the children experienced, with only slightly increased scores for children with good social support and markedly increased depression scores for maltreated children without social support.

It should be mentioned that some studies that aimed to replicate these findings failed to do so (Gillespie et al., 2005; Surtees et al., 2006) or could only partially replicate the findings (Eley et al., 2004; Grabe et al., 2005). Nevertheless, the majority of replication studies have confirmed the gene by environment interaction, supporting an interaction between the 5-HTTLPR, adverse life events, and major depression (see for a recent review Uher \& McGuffin, 2008). Moreover, human neuroimaging studies have shown that individuals carrying the s allele of the 5HTTLPR exhibit a greater stress response in the amygdala in reaction to fearful stimuli compared to individuals homozygous for the I allele (Hariri et al., 2002; Hariri et al., 2005).

In spite of epidemiological studies exploring the interaction between $5-\mathrm{HT}$ and stress, experimental research investigating the interaction between stress and 5-HT vulnerability is scarce and, to our knowledge, the interaction between serotonergic vulnerability and stress in 5-HT vulnerable populations has not yet been studied. However, particularly under acute stress exposure, 5-HT vulnerable subjects may be more prone to develop depressive complaints and may be more susceptible to the negative effects of ATD. In the face of acute stress, the serotonergic system is activated because it plays an important role in regulating HPA axis activity, which is important for stress coping (e.g. Porter et al., 2004). Therefore, transient reduction of 5-HT through ATD may reduce stress 
coping, particularly in 5-HT-vulnerable populations (i.e., individuals with a positive family history of depression, individuals carrying the s allele of the 5-HTTLPR, or individuals with a cognitive reactivity to depression). Furthermore, if transient reduction of 5-HT through ATD induces depressive complaints in 5-HT-vulnerable subjects under acute stress, the question arises of whether the negative effects of acute stress can be counteracted through transiently increasing 5-HT neurotransmission.

One method to increase brain $5-\mathrm{HT}$ is through carbohydrate-rich diets, which have been found to increase the TRP:LNAA ratio (Fernstrom et al., 1973; Curzon, 1985; Markus et al., 1998; Markus et al., 1999). However, the use of carbohydrate-rich diets poses methodological problems, including the lack of placebo-controlled double-blind trials, and the effects on the TRP:LNAA are only modest (Markus, 2008). A different method to enhance TRP availability relative to the other LNAAs, which can easily be used in double-blind placebo-controlled studies, is the use of alpha-lactalbumin protein. Alpha-lactalbumin is a whey protein with the highest TRP content of most protein food sources (Heine et al., 1996). Intake of alpha-lactalbumin has been shown to increase the TRP:LNAA ratio by 50-130\% (Markus et al., 2002; Markus et al., 2005; Merens et al., 2005; Booij et al., 2006) and animal studies have confirmed an increase in brain 5-HT synthesis (Orosco et al., 2004). However, the rise in TRP:LNAA ratio still seems to be insufficient to cause meaningful behavioral changes (e.g. Merens et al., 2005). To date, mood improving effects of alpha-lactalbumin have only been found in stressvulnerable subjects after acute stress exposure (Markus et al., 2000a; Markus et al., 2002), suggesting that the positive effects of 5-HT augmentation with alpha-lactalbumin are exclusively found in vulnerable subjects under conditions when $5-\mathrm{HT}$ is actually needed. Therefore, a new method with a more purified and hydrolyzed protein rich in TRP may have greater effects on the TRP:LNAA ratio (see Chapter 5) and may, therefore, be a better alternative for future studies (see Chapter 6).

\section{CONCLUSION}

Serotonergic vulnerability, a sensitivity of the brain serotonergic system to alterations or disruptions, has been suggested as a risk factor for mood disorders such as depression. Individuals with a family history of depression and individuals with the 5-HTTLPR s allele are thought to suf- 
fer from serotonergic vulnerability, based on serotonergic challenge studies, which may explain their increased risk of developing depression. Serotonergic vulnerability may be innate, as suggested by family and twin studies and 5-HTTLPR genotype studies, but may also be acquired during development or later in life. However, not all individuals with a positive family history of depression and not all s allele carriers develop depression; therefore, we suggest that a positive family history of depression or possession of the $s$ allele may not be risky but may only increase depression susceptibility in the context of stress. After all, stress is suggested as an additional risk factor in the development of depression and it is thought that serotonergic vulnerability, particularly in combination with stress, increases the likelihood of developing depression. Evidence suggests that bi-directional interactions between the neuroendocrine stress mechanisms and the brain serotonergic system are responsible for this increased vulnerability to depression following stressful life events in individuals with a susceptible serotonergic system. Nevertheless, this interaction has not yet been studied experimentally in vulnerable individuals and, therefore, merits research, which may further elucidate the pathophysiology of depression.

\section{AIM OF THE DISSERTATION}

The main aim of this dissertation is to experimentally investigate the interaction between stress and serotonergic vulnerability as a risk factor for depression. The first research question is whether depressionvulnerable individuals indeed exhibit serotonergic vulnerability. The second research question is whether depression vulnerable individuals are susceptible to acute stress exposure, particularly following 5-HT depletion through ATD and whether negative effects of stress may be counteracted by increasing 5-HT availability through TRP augmentation. In the current experiments, different population groups (individuals with a family history of depression, 5-HTTLPR s allele carriers, and individuals with a cognitive reactivity to depression) who are suggested to have an increased risk of depression, possibly due to serotonergic vulnerability, are exposed to serotonergic manipulations (ATD and TRP augmentation) and acute stress to test the serotonergic vulnerability by stress interaction hypothesis. It is hypothesized that individuals with a family history of depression and 5-HTTLPR s allele carriers exhibit stress vulnerability and 
serotonergic vulnerability following ATD demonstrated by mood lowering, negative attention bias, or negative memory bias. Additionally, it is expected that serotonergic vulnerability manifests itself particularly following acute stress. Further, it is hypothesized that individuals with a cognitive reactivity to depression, also thought to have a serotonergic vulnerability, show mood improvements following TRP augmentation and that TRP augmentation may counteract the negative effects of acute stress on mood and cortisol.

\section{METHODOLOGY}

All studies described in this dissertation are conducted with a double-blind placebo-controlled crossover design. In order to explore serotonergic vulnerability under acute stress exposure, the first three studies are conducted with the use of ATD to transiently induce or increase serotonergic vulnerability. ATD is accomplished with a TRP-free collagenprotein (CP) amino acid drink (Blokland et al., 2004; Evers et al., 2005). This ATD method differs from the classic methodology by including a gelatin-based hydrolyzed $\mathrm{CP}$ that contains the entire range of amino acids (except for L-TRP) in the form of peptides. After administration, these peptides are decomposed into amino acids and the mechanism of depletion is identical to the classic ATD method (Blokland et al., 2004; Evers et al., 2005). One advantage of using the CP method is that the placebo mixture may have no meaningful effects on the plasma TRP:LNAA ratio; thereby, preventing unwanted confounding central effects of the placebo that may either under- or overestimate the influence of ATD (Blokland et al., 2004; Evers et al., 2005; Sambeth et al., 2008).

In order to explore the effects of increasing 5-HT availability in depression- (5-HT) vulnerable subjects under acute stress on mood and stress coping, in study four and five, TRP is augmented to transiently increase 5-HT synthesis. Therefore, a TRP-rich hydrolyzed protein is tested to, first, investigate the effects on the TRP:LNAA ratio in comparison to previously used alpha-lactalbumin protein and other TRP sources and, then, explore the effects of 5-HT augmentation on mood and stress coping in individuals with a cognitive reactivity to depression.

To induce acute stress, the Markus-Peters computerized mental arithmetic task (MPA, e.g. Markus et al., 2000a) is used in part of the experiments (Chapters 1, 2, and 3). During the MPA task, subjects must 
solve a specific number of multiple choice mental arithmetic questions under time-constraints while, at the same time, continuous 75,80 , or 85 $\mathrm{dB}$ industrial noise is presented to them through headphones. Subjects are led to believe that the intensity of the noise depends on their performance; however, the criterion is manipulated so that all subjects continue to fail on each trial. In Chapter 6, the Trier Social Stress Test (TSST, Kirschbaum et al., 1993) is used. The TSST, consists of a preparation period $(10 \mathrm{~min})$ followed by a video-taped test period $(10 \mathrm{~min}$ ) during which the subject must give a free speech $(5 \mathrm{~min})$ and perform mental arithmetic ( $5 \mathrm{~min}$ ) in front of an audience. Both stress procedures have frequently demonstrated to induce psychological and physiological stress (e.g. Kirschbaum et al., 1993; Markus et al., 2000b; Markus et al., 2002).

\section{OUTLINE OF THE DISSERTATION}

Chapters 2, 3, and 4 explore the interaction between serotonergic vulnerability, stress, and depression symptoms in individuals with a positive family history of depression $(\mathrm{FH}+)$ and in subjects with different 5 HTTLPR genotypes by the use of ATD. In Chapter 2, the effects of ATD on affective processing before and after exposure to acute stress are tested in individuals with $\mathrm{FH}+$ and controls. It is hypothesized that individuals with $\mathrm{FH}+$ are more prone to the negative affective effects of ATD (due to serotonergic vulnerability), particularly following acute stress exposure, compared to controls. In Chapter 3, the interaction between serotonergic vulnerability, stress, and depression symptoms is investigated in subjects with a genetic vulnerability to depression. In this study, differential effects of 5-HTTLPR genotypes following ATD and acute stress exposure on affective processing are investigated. It is hypothesized that individuals carrying the bi-allelic 5-HTTLPR s allele are more stress- and ATD-susceptible compared to 5-HTTLPR I/I carriers. In Chapter 4, differential effects of tri-allelic 5-HTTLPR genotypes on mood, memory, and attention bias following ATD and stress exposure are investigated. Mood lowering and negative attention and memory bias are expected following ATD and stress exposure, particularly in 5-HTTLPR s allele carriers.

Chapters 5 and 6 explore the effects of 5-HT augmentation on mood and stress coping in healthy non-vulnerable and healthy depres- 
sion-vulnerable subjects. In Chapter 5, it is tested whether a newly developed TRP-rich hydrolyzed protein has greater effects on the plasma TRP:LNAA ratio and mood than the previously used intact alphalactalbumin protein. In Chapter 6, the interaction between serotonergic vulnerability, stress, and depression symptoms in individuals with a cognitive reactivity (CR) to depression is investigated. It is hypothesized that subjects with high CR due to serotonergic vulnerability may particularly benefit from increased 5-HT availability, especially under acute stress. Therefore, mood and cortisol responses following TRP augmentation with a TRP-rich hydrolyzed protein and acute stress in healthy subjects with high and low CR to depression are measured. Beneficial effects of 5-HT augmentation on mood and stress coping, particularly in individuals with high $\mathrm{CR}$ are expected.

Chapter 7 gives an overview and a general discussion of the main findings of this dissertation. Further, suggestions for future research are provided. 


\section{CHAPTER 2}

Effects of acute tryptophan depletion on affective processing in first-degree relatives of depressive patients and controls after exposure to uncontrollable stress

Based on:

Firk, C., Markus, C.R. (2008). Effects of acute tryptophan depletion on affective processing in first-degree relatives of depressive patients and controls after exposure to uncontrollable stress. Psychopharmacology 199, 151-160. 


\section{ABSTRACT}

\section{Rationale}

Individuals with a family history of depression may be more likely to develop depression due to an innate vulnerability of their serotonergic system. However, even though serotonergic vulnerability may constitute a risk factor in the development of depression it does not seem to be sufficient to cause a depressive episode. Based on previous data, it is suggested that stress may be a mediating factor.

\section{Objectives}

This study examined the role of serotonin $(5-\mathrm{HT})$ in stress coping in individuals with or without a family history of depression.

Methods

Nineteen healthy first degree-relatives of depressive patients $(\mathrm{FH}+)$ and 19 healthy controls without a family history of depression (FH-) were tested in a double-blind placebo-controlled design for affective processing under acute stress exposure, following acute tryptophan depletion (ATD) or placebo.

\section{Results}

Significant negative effects were found of stress on affective processing in $\mathrm{FH}-$ and $\mathrm{FH}+$. In addition, $\mathrm{FH}$ - responded slower to positive words after stress only following ATD, whereas $\mathrm{FH}+$ responded marginally slower under stress already after placebo and before stress following ATD.

\section{Conclusion}

Acute stress exposure reduces positive affective bias; supporting the role of stress as an important predecessor in the development of depression. Further, $\mathrm{FH}+$ may be more susceptible than $\mathrm{FH}$ - to the negative effects of stress as well as to the negative effects of ATD. The results support the assumption that the $5-\mathrm{HT}$ system is involved in stress resilience and may be more vulnerable in first degree relatives of depression. 


\section{INTRODUCTION}

First-degree relatives of depressive patients have a two- to threefold increased risk of developing depression (Sullivan et al., 2000; Kelsoe). Although environmental factors play a role, adoption and twin studies indicate that the heritability ranges between $31 \%$ and $41 \%$ (Sullivan et al., 2000). Although the neurobiological equivalent of this genetic predisposition remains unclear, the brain serotonergic system seems to be involved (Owens \& Nemeroff, 1994; Maes \& Meltzer). Evidence comes from studies reporting lower plasma availability of the serotonin (5-HT) precursor tryptophan (TRP) for the brain, reduced cerebrospinal fluid (CSF) concentration of the 5-HT metabolite 5-hydroxyindoleacetic acid (5-HIAA) and decreased platelet 5-HT uptake in depression, suggesting diminished brain 5-HT function (Maes \& Meltzer, 1995; Neumeister et al., 2004c).

Acute tryptophan depletion (ATD) is commonly used to study serotonergic vulnerability (Young et al., 1985; for a review see Fusar-Poli et al., 2006). The intervention reduces brain 5-HT through intake of a TRPfree amino acid mixture which reduces tryptophan (TRP) relative to the sum of the other large neutral amino acids (LNAA's) with which TRP competes for brain uptake (i.e. Fernstrom \& Wurtman, 1971; Gessa et al., 1974; Moja et al., 1991; Maes \& Meltzer, 1995). Reduced 5-HT neurotransmission after ATD is indicated by decreased brain 5-HT synthesis and release as well as by lower concentrations of cerebrospinal fluid 5hydroxyindoleacetic acid (5-HIAA) (Nishizawa et al., 1997; Carpenter et al., 1998; Williams et al., 1999).

ATD is found to reverse antidepressant-induced remission (Delgado et al., 1990; Delgado et al., 1999; Booij et al., 2005) and induces depressive symptoms in remitted depressive patients (Moreno et al., 2000; Neumeister et al., 2004b; Hayward et al., 2005; Moreno et al., 2006; Booij \& Van der Does, 2007), whereas in healthy subjects no or only modest effects are found (e.g. Benkelfat et al., 1994; Bhatti et al., 1998; Klaassen et al., 1999; Evers et al., 2005; Fusar-Poli et al., 2007; Ruhe et al., 2007). However, depressogenic effects of ATD seem to be mediated by family history for depression. Healthy subjects with a positive family history $(\mathrm{FH}+)$ of depression show greater depressed mood after ATD than healthy controls without a family history (FH-) (Benkelfat et al., 1994; 
Klaassen et al., 1999; Neumeister et al., 2002; Sobczak et al., 2002a; van der Veen et al., 2007). Furthermore, mood lowering effects of ATD may depend on the 5-HT transporter genotype, a gene-linked polymorphic region (5-HTTLR) with two functional variants (Neumeister et al., 2002; Neumeister et al., 2006; Roiser et al., 2007; Walderhaug et al., 2007) that has been shown to modulate the vulnerability to depression (Caspi et al., 2003). These findings support the assumption of serotonergic vulnerability as a risk factor for depression.

Even though serotonergic vulnerability may constitute a likely risk factor in the development of depression, it does not seem to be the sole contributor. Recent studies revealed that stress may be an important mediating factor. Stressful life events often precede the onset of depression (Brown et al., 1987; Heim \& Nemeroff, 2001; Van Praag, 2004) and individuals with a genetic $5-\mathrm{HT}$ vulnerability respond more readily to stressful life events with depressive feelings than individuals without a genetic vulnerability (Caspi et al., 2003). Furthermore, there is considerable evidence for complex interactions between the serotonergic system and neuroendocrine stress mechanisms (Van Praag, 2004) and 5-HT is involved in the initiation and termination of the stress response (Lefebvre et al., 1992; Dinan, 1996; Fuller). Acute stress increases brain 5-HT turnover (e.g. De Kloet et al., 1982; De Kloet et al., 1983; Davis et al., 1995) as a biological mechanism for stress adaptation (Nuller \& Ostroumova, 1980; Van Praag et al., 2004), whereas dysfunctional brain 5-HT is found to reduce HPA function and stress adaptation (Maes et al., 1991; Seckl \& Fink, 1991). In addition, brain 5-HT augmentation is found to reduce the negative effects of stress on cortisol stress-responses and depressive symptoms in healthy but stress- susceptible subjects compared to controls (Markus et al., 2000a; Markus et al., 2002). In accordance, Richell et al. (Richell et al., 2005) reported that even healthy subjects are susceptible to the mood-lowering effects of stress exposure following ATD.

Based on these previous findings, $\mathrm{FH}+$ individuals are thought to be prone to the negative affective effects of stress due to serotonergic vulnerability. In addition, this may even be more profound after ATD.

Depression is associated with reduced attention, memory and executive functioning (Elliott et al., 1996; Porter et al., 2003; Tavares et al., 2003; Paelecke-Habermann et al., 2005); among which attention bias towards negative information has frequently been demonstrated (e.g. Lim \& Kim, 2005; Rinck \& Becker, 2005). Depressed patients respond 
slower to happy words compared to sad words during affective go/nogo tasks (Murphy et al., 1999; Erickson et al., 2005) and this was also observed in healthy individuals following ATD (Murphy et al., 2002).

The present study investigated whether first-degree relatives of depressive patients $(\mathrm{FH}+)$, as compared with subjects without a family history of depression (FH-), are more prone to the negative effects of stress exposure and ATD on affective processing. Based on previous data (Murphy et al., 2002), it is hypothesized that stress, particularly after ATD, would slow down responses to positive words particularly in individuals with $\mathrm{FH}+$.

\section{METHODS}

\section{Subjects}

Maastricht University students $(n=200)$ completed a questionnaire package concerning personal details. Students reporting having at least one first degree-relative diagnosed with major depression were invited for a personal interview, as well as students reporting no first and second degree relative with a depressive disorder. To assess $\mathrm{FH}$, all participants were interviewed by a trained psychologist with an abbreviated version of the Family History Research Diagnostic Criteria (FHRDC) (Endicott et al. 1975). In addition, participants meeting the $\mathrm{FH}+$ inclusion criteria were asked whether relatives could be contacted for confirmation.

Nineteen healthy first degree-relatives of depressive patients $(\mathrm{FH}+)$ and 19 healthy controls without a family history of depression (FH-) were selected for the experiment. A structured psychiatric interview (MINI) (Sheehan et al., 1994) was carried out to exclude psychiatric disorders. Furthermore, the Symptom Checklist SCL-90 (Arrindell \& Ettema, 1986) and the Beck Depression Inventory (BDI) (Beck et al., 1961) were filled in to verify the absence of depressive and general psychopathologic symptomatology. The $\mathrm{FH}$ - group and the $\mathrm{FH}+$ group did not differ with respect to sex, age, BMI and BDI and SCL-90 scores (all $p>$.05). Demographic characteristics are presented in Table 1.

Participants were excluded if they reported chronic and current illness; history of psychiatric or medical illness; medication use; metabolic-, hormonal- or intestinal diseases; irregular diets; or deviant eating habits 
and excessive alcohol or drug use. Participants' health was checked with standardized medical questionnaires that were evaluated by a trained doctoral-level psychologist under the supervision of a medical doctor.

Participants included in the study revealed normal body-mass indexes (BMI in $\mathrm{kg} / \mathrm{m}^{2}$ between 19-26), were non-smokers and were requested not to use alcohol or any kind of drugs before and during the study. Inclusion criteria for $\mathrm{FH}+$ were the presence of at least one firstdegree relative with major depression according to the DSM-IV criteria, whereas inclusion criteria for $\mathrm{FH}$ - include absence of a first- or seconddegree relative with major depression.

The study was approved by the Medical Ethics Committee of the Academic Hospital Maastricht and complied with the requirements of the European Council of Good Clinical Practice (GCP) adopted by the $52^{\text {nd }}$ World Medical Association General Assembly, Edinburgh, Scotland (October, 2000). All subjects gave their informed consent and were paid 125 Euros for participation.

Table 1. Demographic characteristics of the $\mathrm{FH}+$ group and $\mathrm{FH}$ - group. Values are mean (SD).

\begin{tabular}{lll}
\hline & \multicolumn{2}{c}{ Family history (FH) } \\
\cline { 2 - 3 } & $\mathrm{FH}+$ & $\mathrm{FH}-$ \\
\hline Women & 15 & 14 \\
Men & 4 & 5 \\
Age & $20.5(2.1)$ & $22.1(3.6)$ \\
BMI & $21.7(2.2)$ & $23.1(2.5)$ \\
SCL-90 & $19.9(17.9)$ & $26.4(23.6)$ \\
BDI & $3.6(3.6)$ & $5.4(4.2)$ \\
\hline
\end{tabular}

\section{Design}

A placebo-controlled, double-blind, crossover design was used. During two experimental sessions, subjects were monitored for affective processing before and after acute stress exposure either following intake of TRP-free (ATD) or a TRP-containing placebo (PLC) amino acid mixture. The order of presentation of the ATD and PLC condition was counterbalanced within groups and both experimental sessions were separated by at least one week. Female subjects were tested in the follicular phase of their menstrual cycle or when actually taking oral contraceptives. 


\section{Procedure}

Eligible participants attended a briefing at Maastricht University to receive information about the study and to be scheduled for the experiment.

On each experimental morning, two subjects arrived at the laboratory at 08:30 am and 10:00 am, respectively. Subjects fasted overnight; only water or tea without sugar was permitted. After arrival, a first blood sample was taken followed by a first version of the affective go/no-go (AGNG) task to make subjects familiar with the test condition. Then, a first measurement of vegetative side effects was conducted followed by administration of the amino acid mixture $\left(\mathrm{t}_{0}\right)$. Four and a half hours later $\left(t_{4.5}\right)$, a second blood-sample was taken followed by a second measure of vegetative side effects. Then ( $\left.t_{5}\right)$ participants conducted a second version of the AGNG task followed by the stress task. After completion of the stress task, a third version of the AGNG task was administered.

Between intake of the amino acid mixture and exposure to laboratory tasks, the subjects were able to study or to read magazines in a separated private room. They had free access to water and decaffeinated tea. Two hours after administration of the amino acid mixture, they received a standardized protein-poor lunch as previously used in ATD studies (Riedel et al., 1999; Sobczak et al., 2002a; Sobczak et al., 2002b). At the end of each test day, subjects received a high protein snack and bananas, which are natural sources of L-TRP to facilitate a quick recovery from possible negative effects of ATD.

\section{Acute tryptophan depletion}

A reduction in brain $5-\mathrm{HT}$ was accomplished by ATD through the use of a TRP-free collagen-protein (CP) amino acid drink (Blokland et al., 2004; Evers et al., 2005). To obtain a drinkable mixture, $100 \mathrm{~g}$ of the protein powder was mixed with $200 \mathrm{ml}$ of tap water and $20 \mathrm{ml}$ syrup. The placebo mixture was identical in composition but $1.2 \mathrm{~g}$ I-TRP (Sigma, Zwijndrecht; The Netherlands) was added. See Table 2 for the amino acid composition of the different conditions (Evers et al., 2005). 
Table 2. Composition (grams) of the gelatin-based protein (all values are $\mathrm{g}$ per $100 \mathrm{~g}$ of each mixture)

\begin{tabular}{lll}
\hline & ATD & PLC \\
\hline Phenylalanine & 1.9 & 1.9 \\
Tyrosine & 0.4 & 0.4 \\
Valine & 2.1 & 2.1 \\
Leucine & 3 & 3 \\
Isoleucine & 1.4 & 1.4 \\
Tryptophan & 0.1 & 1.3 \\
Serine & 3.1 & 3.1 \\
Glycine & 22.5 & 22.5 \\
Histidine & 0.5 & 0.5 \\
Arginine & 8.8 & 8.8 \\
Threonine & 1.1 & 1.1 \\
Alanine & 9.3 & 9.3 \\
Proline & 13.3 & 13.3 \\
Methionine & 0.6 & 0.6 \\
Cystein & 0.2 & 0.2 \\
Lysine & 3.6 & 3.6 \\
Hydroxyproline & 12.1 & 12.1 \\
Hydroxylysine & 1.4 & 1.4 \\
Aspartic acid + asparagines & 9.3 & 9.3 \\
Glutamic acid + glutamine & 5.2 & 5.2 \\
\hline
\end{tabular}

This ATD method differs from the classic methodology by including a gelatin-based hydrolyzed $\mathrm{CP}$ that contains the entire range of amino acids (except for L-TRP) in the form of peptides. After administration, these peptides are decomposed into amino acids and the mechanism of depletion is identical to the classic ATD method. (Blokland et al., 2004; Evers et al., 2005).

\section{Stress exposure}

The Markus-Peters computerized mental arithmetic task (MPA) was used as an uncontrollable stress situation. Subjects were given 8 successive 1-min trials during which they had to solve a specific number of multiple choice mental arithmetic problems (the criterion) under time constraints, while exposed to continuous 75,80 or $85 \mathrm{~dB}$ industrial noise 
presented through headphones. They were led to believe that the intensity of the noise depended on their performance; if they failed the criterion, noise intensity was chosen by the computer during the next trial; if they met the criterion, they could choose the intensity of the noise. In fact, the criterion was always set at one sum above what subjects could manage as calculated from the average time per sum needed on previous trials. This task has been demonstrated to induce psychological and physiological stress (Markus et al., 1998; Peters et al., 1998; Markus et al., 2000a; Markus et al., 2000b).

\section{Affective go/no-go paradigm}

A modified version of the AGNG task described by Murphy et al. (1999) was used to detect affective attentional bias. In this task, happy and sad words are presented on the screen one-by-one for $300 \mathrm{~ms}$, followed by an inter-stimulus interval of $900 \mathrm{~ms}$ during which participants must make or withhold a response depending on word valence. The task comprised 2 practice and 12 experimental blocks; each containing 9 happy words and 9 sad words. Subjects were instructed to respond either to happy or sad words before each block and to respond as quickly as possible. Every two blocks, the targets and the distractors changed; words that were previously targets became distractors and vice versa (SSHHSSHHSSHHSS or HHSSHHSSHHSSHH). Due to this arrangement, shift blocks and non-shift blocks could be studied. The 27 happy words and 27 sad words were derived from previous studies (e.g. Lim \& Kim, 2005; Rinck \& Becker, 2005) and were matched on frequency, word length and valence. Every word was presented twice as target and twice as distractor; once in a shift block and once in a non-shift block.

\section{Vegetative side effects}

In order to measure possible side effects of the amino acid mixtures, a list (5-point scales) of vegetative side effects was completed before and 4.5 hour $\left(t_{4.5}\right)$ after intake. The list contained the following items: feeling cold, feeling hot, dizziness, perspiration, blurred vision, nausea, palpitations, dry mouth and abdominal complaints. 


\section{Biochemical Analyses}

Blood samples were collected in $5 \mathrm{ml}$ vacutainer tubes containing sodium heparin for amino acids and were centrifuged at $5000 \mathrm{rpm}$ for 10 min at $4^{\circ} \mathrm{C}$. Subsequently, the supernatants were directly stored at $-80^{\circ} \mathrm{C}$ until analysis. Before storage, the supernatant for amino acid determination $(100 \mu l)$ were mixed with $4 \mathrm{mg}$ sulfasalicyl acid. Analyses were conducted with HPLC, making use of a 2-3 $\mu \mathrm{m}$ Bischof Spherisorb ODS II column. The plasma TRP ratio was calculated by dividing the TRP concentration by the sum of the other large neutral amino acids, i.e. valine, isoleucine, leucine, tyrosine and phenylalanine.

\section{Statistical analysis}

The main research questions were analyzed by repeated measures analyses of variance (ANOVAs) by using the General Linear Model (GLM: SPSS 12.0 for Windows) with one between-subjects factor Family history $(\mathrm{FH}+$ vs. $\mathrm{FH}-)$ and the within-subjects factors Treatment (ATD vs. PLC), Stress (pre-stress vs. post-stress) or Time $\left(t_{0}\right.$ vs. $\left.t_{4.5}\right)$ on the several dependent measures. Further, in the analyses of the AGNG performance Target valence (sad vs. happy) and Shift (shift vs. non-shift condition) were added as within-subjects factors. Although we counterbalanced for Order and Gender, these factors were preliminary taken as covariates. However, since none of these factors contributed to (or changed) any of our findings, Order of Treatment and Gender were left out of the final analyses. All statistics are evaluated at a significance level of 5\% (twotailed).

\section{RESULTS}

\section{Plasma amino acids (TRP:LNAA ratio)}

Repeated measures analysis of variance with $\mathrm{FH}(\mathrm{FH}-\mathrm{vs}$. $\mathrm{FH}+$ ) as between-subjects factor and Treatment (ATD vs. PLC) and Time ( $t_{0}$ vs. $\left.t_{4.5}\right)$ as within-subjects factors were carried out for total plasma TRP concentrations and for the TRP:LNAA ratio. For TRP concentrations a significant 
Treatment $X$ Time interaction was found $[F(1,36)=156.57, p<.001]$ reflecting a decrease from $t_{0}$ to $t_{4.5}$ by $62 \%$ after ATD and an increase from $t_{0}$ to $t_{4.5}$ by $13 \%$ after PLC administration (see Figure 1). Analysis of the plasma TRP:LNAA ratio revealed a significant Treatment $X$ Time interaction $[F(1,36)=158.77, p<.001]$. As indicated in Figure 1 , there was a $65 \%$ decline in plasma TRP:LNAA after ATD and an increase from $t_{0}$ to $t_{4.5}$ by $8 \%$ after PLC. No other main or interaction effects were found including $\mathrm{FH}$.

\section{Total TRP}

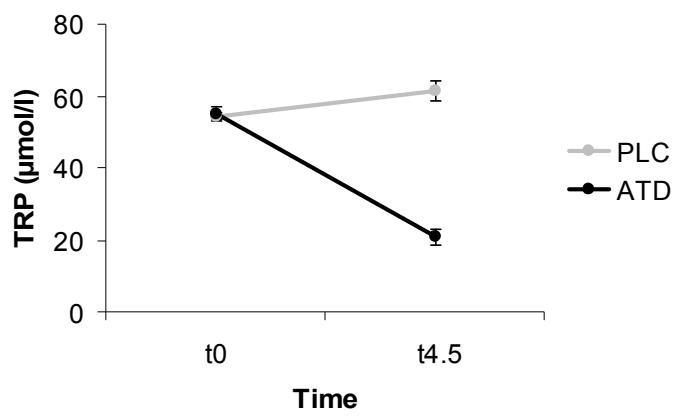

\section{TRP:LNAA ratio}

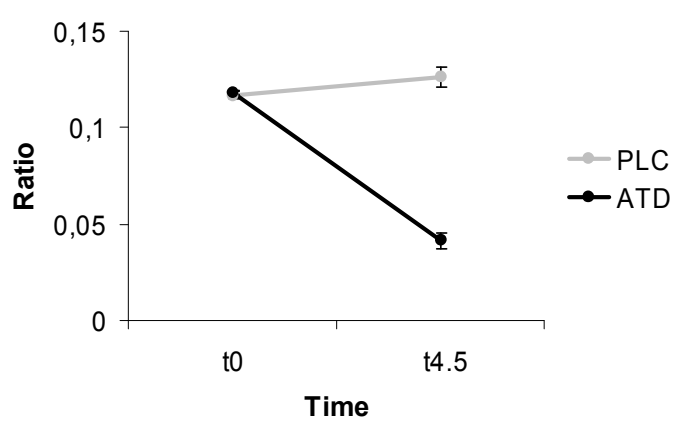

Figure 1. Plasma total TRP concentrations and TRP:LNAA ratio for the ATD and PLC condition.

Vegetative side effects

Repeated measures analysis of variance with $\mathrm{FH}$ ( $\mathrm{FH}-\mathrm{vs}$. $\mathrm{FH}+$ ) as between-subjects factor and Treatment (ATD vs. PLC) and Time ( $t_{0}$ vs. $\left.t_{4.5}\right)$ 
as within-subjects factors on the total score of vegetative side effects did not reveal any significant main or interaction effects.

\section{Affective go/no-go performance}

Mean values and standard deviations are presented in Table 3. Repeated measures ANOVAs were carried out with $\mathrm{FH}(\mathrm{FH}-\mathrm{vs}$. $\mathrm{FH}+$ ) as between-subjects factor and Treatment (ATD vs. PLC), Target valence (sad vs. happy), Shift (shift vs. non-shift condition) and Stress (pre-stress vs. post-stress) as within-subjects factors on reaction time, errors and omissions. To investigate changes from baseline to pre-stress (to explore acute effects of treatment), we repeated the statistical analyses and replaced the WS factor Stress (pre-stress vs. post-stress) with the WS factor Time (baseline vs. pre-stress). However, as we did not find any effect of Treatment, these analyses are not described. Further, no baseline differences were found between test days.

Reaction Time (RT). Analysis of RT data revealed a significant Valence $X$ Stress interaction $[F(1,36)=5.42, p=.026]$ reflecting increased $\mathrm{RTs}$ for happy words post-stress compared to pre-stress [ $t(37)=2.09, p<.0 .043]$ but not for sad words $[t(37)=1.39, p=0.17]$ (Figure 2). However, this interaction was qualified by a significant 5-way $F H X$ Treatment $X$ Stress $X$ Valence $X$ Shift interaction $[F(1,36)=3.99, p=0.05]$. Further analysis for the shift and non-shift condition separately revealed a significant $F H X$ Treatment $X$ Stress $X$ Valence interaction for the non-shift condition only $[F(1,36)=6.36, p=0.016]$. In the non-shift condition, there was a significant $\mathrm{FH} \times$ Treatment $X$ Stress interaction for happy words only $[F(1,36)=6.69, p=0.014])$. As visualized in Figure 3, the $\mathrm{FH}$ - group showed slower RTs post-stress compared to pre-stress only following ATD $[t(18)=2.57, p=0.019]$ but not after PLC $[t(18)<1]$, whereas the $\mathrm{FH}+$ group showed slower RTs post-stress compared to pre-stress independent of treatment and slower RTs already following ATD (before stress onset). These latter changes in the $\mathrm{FH}+$ group, however, did not approach significance by further post-hoc testing [P between 0.07 0.11). 


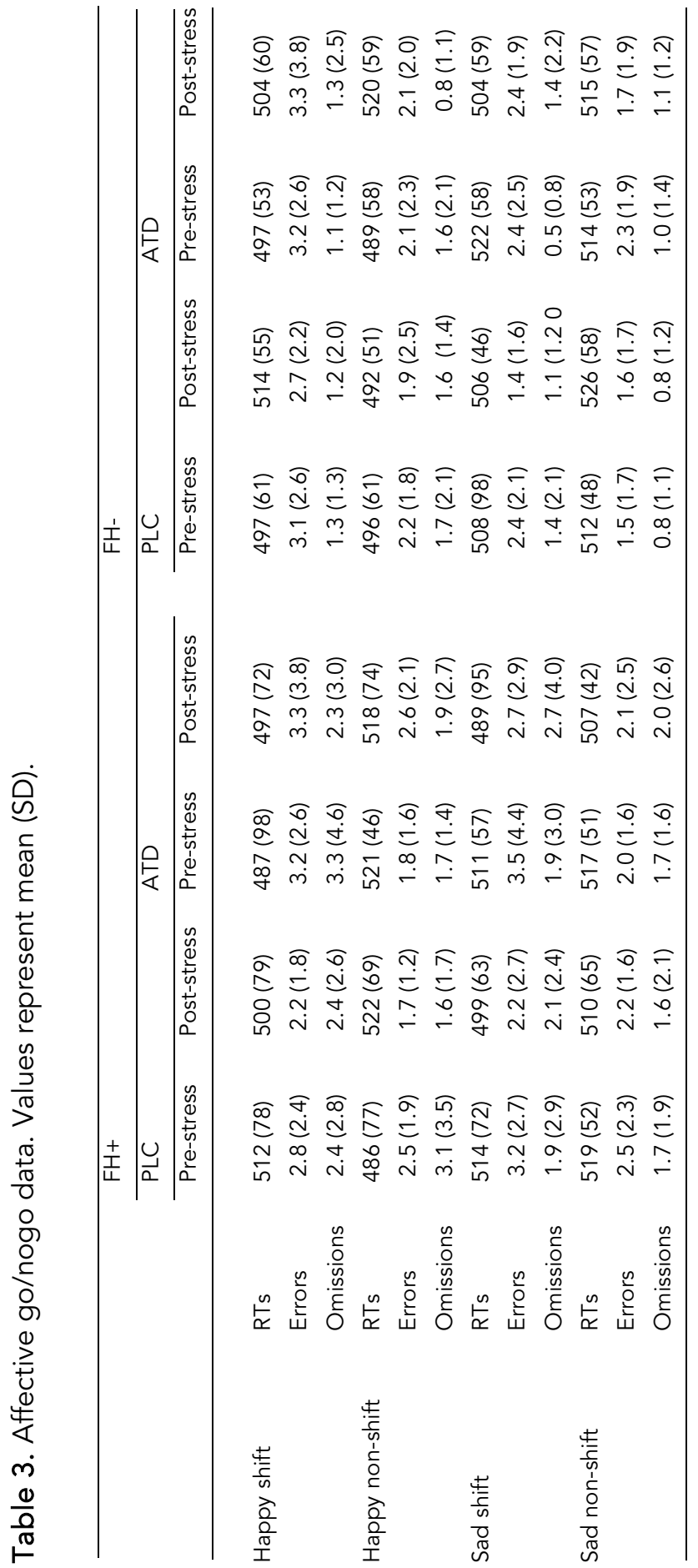




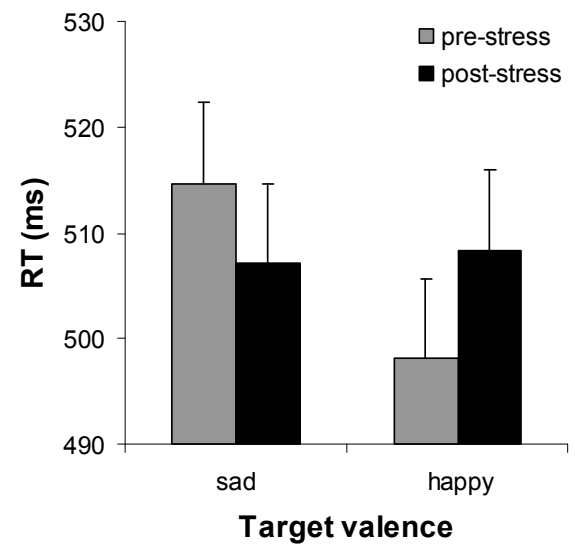

Figure 2. Mean RTs (SE) for happy and sad targets before and after stress exposure collapsed over treatment and family history.

FH+

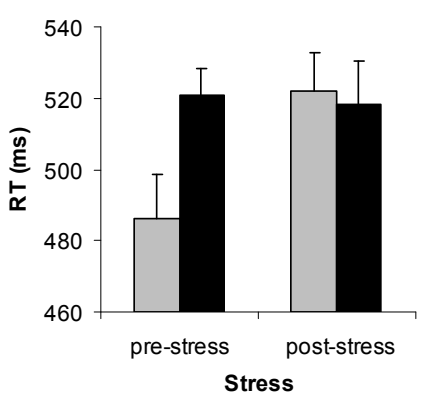

FH-

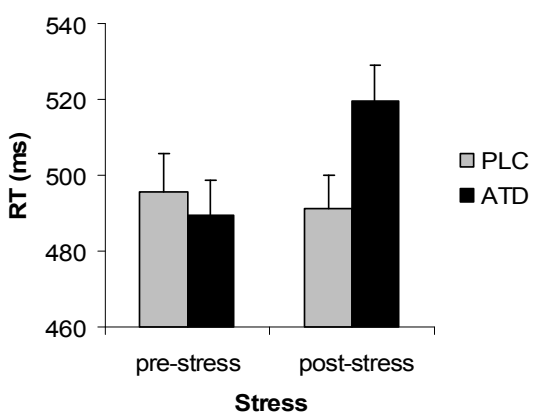

Figure 3. Mean RTs (SE) for happy non-shift targets for the FH+ group and the FH- group.

Errors. Analysis of error data revealed a main effect of Shift $[F(1,36)=18.81, p<0.001]$, indicating significant more errors during shift blocks compared to the non-shift blocks. Analysis also revealed a main effect of Stress $(F(1,36)=8.42, p=0.006)$, indicating a decrease in the number of errors post-stress compared to pre-stress. There were no effects of $\mathrm{FH}$, Treatment or Valence.

Omissions. Analysis of omission data revealed a significant Stress by Valence interaction $[F(1,36)=7.57, p=0.009]$, indicating that significant 
more omissions were made pre-stress compared to post-stress for happy words [ $t(37)=2.31, p=0.027]$ but not for sad words $[t(37)=1.16, p$ $=0.25]$. There were no effects of FH or Treatment.

\section{DISCUSSION}

The goal of the present study was to assess affective processing in individuals with a positive $(\mathrm{FH}+)$ or negative $(\mathrm{FH}-)$ family history of depression following ATD or placebo under acute stress exposure. ATD lowered the plasma TRP:LNAA ratio by $65 \%$, which is comparable with previous studies using the collagen-protein (Evers et al., 2005) or classic ATD mixture (Van der Does, 2001).

Although psychological or physiological stress responses were not measured in the current study, significant stress-induced emotional, cognitive, hormonal and electrophysiological changes have been reported with the MPA task (e.g. Peters et al., 1998; Markus et al., 2002). Furthermore, reaction times significantly decreased after the stress task, further supporting that stress was successfully induced in the current study.

Analysis of affective processing revealed a stress by valence interaction reflecting reduced responsiveness to happy words after acute stress exposure. Previous findings already demonstrated slowed or diminished responses to happy words in depressed patients compared to healthy controls (Murphy et al., 1999; Deveney \& Deldin, 2004; Erickson et al., 2005), which may reflect lower mood and subsequent increased interference from sad distractors. Current findings indicate that a positive affective bias normally found in healthy individuals may also be diminished by acute stress exposure, which may be due to a stress-induced lowering of mood (van der Veen et al., 2007). This further supports the hypothesis of stress as an important predecessor in the development of depression (Brown et al., 1987; Heim \& Nemeroff, 2001; Van Praag, 2004). Current data further suggest that the negative effects of stress on (reducing) positive affective bias may depend on family history of depression and may be influenced by ATD and task-shifting. The FH-group showed stress-induced slowed responses to happy words in non-shift blocks only following ATD, whereas the FH+ group roughly seemed to exhibit such reductions already after ATD (which was not found after PLC) as well as after stress following PLC. 
The present findings suggest that acute stress induces a negative affective bias and that $\mathrm{FH}$ - subjects may be more stress-resilient than $\mathrm{FH}+$ subjects and may become susceptible to stress especially after ATD. Interestingly, although task shifting requires more cognitive flexibility (e.g. Monsell, 2003), the negative affective bias (slowed responses to happy words following stress and ATD) could only be seen for the non-shift blocks but not for the shift-blocks. Although hypothetical, the increased negative affective bias following stress as well as ATD may be less profound the more attention or alertness is required (shift blocks) and may be increased in situations in which less attention is required (non-shift blocks). Previous ATD studies with healthy subjects revealed mixed results; either reporting negative affective bias following ATD for shift and non-shift blocks (Murphy et al., 2002) or no effects at all (Rubinsztein et al., 2001; Roiser et al., 2007). Current data may explain these inconsistent findings by the mediating influence of stress. Individuals with a family history of depression may already be prone to the negative effects of ATD and may also be more negatively affected by stress in the absence of ATD. These findings should of course be interpreted with caution since they appear to be rather modest (also depending on task-shifting and valence) and do not remain significant after repeated post-hoc testing in the separated small $\mathrm{FH}+$ group. Yet, they nicely comply with -and elaborate on- previous findings and suggestions of an innate serotonergic vulnerability in $\mathrm{FH}+$ (Benkelfat et al., 1994; Klaassen et al., 1999; Sobczak et al., 2002a; Sobczak et al., 2002b). Hence, 5-HT plays an important role in stress coping and clear interactions appear between 5-HT and the neuroendocrine stress system (Porter et al., 2004). Acute stress increases 5-HT neurotransmission (De Kloet et al., 1982; De Kloet et al., 1983; Davis et al., 1995), which promotes stress adaptation by mediating negative feedback control of cortisol on the HPA axis (Nuller \& Ostroumova, 1980; Van Praag, 2004). Increased 5-HT under stress will be diminished after ATD and subsequently may increase stress vulnerability. Richell et al. (2005) reported greater negative mood after stress in healthy subjects following ATD that was attributed to reduced function of the 5-HT mediated resilience system and subsequent enhanced stress susceptibility. In the studies by Markus et al. (2000a; 2002), 5-HT augmentation was found to enhance resilience to stress only in chronically stressed (healthy) subjects probably by compensatory stress-induced 5HT receptor sensitization. Whereas 5 -HT challenge may particularly im- 
prove stress coping in 5-HT vulnerable subjects, ATD may lower stress coping also in non-vulnerable subjects due to a drastic depletion in brain TRP and subsequent 5-HT function.

One limitation of the present study is that we did not include mood changes. It has been suggested that ATD- induced changes in affective processing are mediated by reduced mood (van der Veen et al., 2007) which may also hold for stress-induced changes. However, it remains questionable whether ATD- or stress-induced changes in affective processing are necessarily mediated by subjective mood changes. Murphy et al (2002) reported a negative affective bias on the AGNG task following ATD in healthy volunteers but did not find any changes in mood. Therefore, measuring affective processing may be a more sensitive method to measure stress- or ATD related changes. Yet, in further studies it would, nevertheless, be interesting to include changes in explicit mood experiences as an additional affective measure. A second limitation may be that we did not contact family members to confirm diagnoses, however, participants were interviewed by a trained psychologist and to increase reliability all participants were asked whether relatives could be contacted to confirm diagnoses.

In conclusion, acute stress exposure reduces positive affective bias supporting the role of stress as an important predecessor in the development of depression. However, these negative effects of stress may depend on family history of depression: $\mathrm{FH}+$ may be more susceptible to the negative effects of stress and ATD on affective processing than $\mathrm{FH}$-. Nevertheless, the tentative explanation that $\mathrm{FH}+$ are more prone to stress and ATD due to an innate serotonergic vulnerability merits further research. 



\section{CHAPTER 3}

Effects of 5-HTTLPR genotype and acute tryptophan depletion on affective processing following acute stress exposure

Firk, C., Markus, C.R. (in preparation). Effects of 5-HTTLPR genotype and acute tryptophan depletion on affective processing following acute stress exposure. 


\section{ABSTRACT}

\section{Rationale}

Epidemiological studies propose an association between the short allele (s) of the serotonin transporter genotype polymorphism (5-HTTLPR) and depressive reaction to stressful life events. Further, the 5-HTTLPR s allele may confer increased vulnerability to acute tryptophan depletion (ATD). Objective

The aim of the present study was to investigate the effects of ATD in subjects with different 5-HTTLPR genotypes before and after stress exposure on affective processing.

\section{Methods}

Thirty-eight subjects were genotyped for 5-HTTLPR and on two experimental morning sessions affective processing was monitored before and after exposure to acute stress either following ATD or placebo (PLC). Results

We found a genotype by stress by shift interaction on the affective go/no-go task indicating fewer omissions for targets on non-shift blocks post-stress compared to pre-stress for individuals with the I/I genotype. Conclusion In contrast to previous findings, 5-HTTLPR genotype did not mediate the effect of ATD on affective processing but long-allele homozygotes were less affected by acute stress relative to short s/s-allele homozygotes. 


\section{INTRODUCTION}

The neurotransmitter serotonin (5-HT) has been implicated in the patophysiology of depression and in particular the serotonin transporter (5HTT) seems to be involved (Owens \& Nemeroff, 1994). Interestingly, the human 5-HTT gene, located on chromosome 17q11.1-q12 (Lesch et al., 1994), contains a 5-HTT-linked polymorphic region (5-HTTLPR) with two functional variants: the short (s) and long (I) allele form (Heils et al., 1996; Greenberg et al., 1999). The s allele has lower transcriptional efficiency than the I allele resulting in lower serotonin uptake activity (Greenberg et al., 1999). The $s$ allele has been associated with increased risk for depression (Collier et al., 1996; Joiner et al., 2003; Gonda et al., 2005; Gonda et al., 2006), poorer responses to antidepressant effects of SSRI's (Smeraldi et al., 1998; Serretti et al., 2007), increased vulnerability to tryptophan depletion (Neumeister et al., 2002; Roiser et al., 2006) and abnormal emotional processing (Hariri et al., 2002; Roiser et al., 2005; Marsh et al., 2006).

Recently, epidemiological studies have demonstrated a positive association between the s allele 5-HTTLPR and depressive reaction to stressful life events (Caspi et al., 2003; Jacobs et al., 2006; Wilhelm et al., 2006), which is in line with common findings of stress as a perpetrator of depression and complex interactions between the serotonergic system and neuroendocrine stress mechanisms (Van Praag, 2004; Firk \& Markus, 2007). However, the interaction between acute stress, 5-HTTLPR and depressive symptoms has not yet been experimentally investigated.

Besides depressive mood, depression is often characterized by attention bias towards negative information (e.g. Lim \& Kim, 2005; Rinck \& Becker, 2005). For example, depressed patients show slowed responding to happy words compared to sad words on affective go/no-go tasks (Murphy et al., 1999; Erickson et al., 2005). This was also observed for healthy individuals following acute tryptophan depletion (ATD), suggesting that serotonergic factors are particularly involved in this negative "affective bias" seen in depression (Murphy et al., 2002).

Based on these findings, the main research question of the present study was whether 5-HTTLPR genotype mediates the affective effects of ATD and whether this is more profound following acute stress exposure. 


\section{METHODS}

\section{Subjects}

Thirty-eight healthy Dutch University students participated in the experiment. Fifteen (39,5\%) participants showed the I/I genotype, 14 $(36,8 \%)$ the $\mathrm{s} / \mathrm{l}$ genotype and $9(23,7 \%)$ showed the s/s genotype. All of them were included in a previous report describing the effects of family history (Firk \& Markus, 2008). The genotype groups did not differ with respect to sex, age, BDI (Beck Depression Inventory, Beck et al., 1961), BMI (Body Mass Index) and FH (Family History) (all $p<.05$ ). Demographic characteristics can be found in Table 1. The study was approved by the Medical Ethics Committee of the Academic Hospital Maastricht and complied with the requirements of the European Council of Good Clinical Practice (GCP) adopted by the $52^{\text {nd }}$ World Medical Association General Assembly, Edinburgh, Scotland (October, 2000). All subjects gave their informed consent and were paid $125 €$ for participation.

Table 1. Demographic characteristics; positive family history $(\mathrm{FH}+)$, body mass index (BMI), Beck Depression Inventory (BDI); mean (SD)

\begin{tabular}{llll}
\hline & Genotype & & \\
\cline { 2 - 4 } & $\mathrm{s} / \mathrm{s}$ & $\mathrm{s} / \mathrm{l}$ & $1 / \mathrm{I}$ \\
\hline $\mathrm{N}$ & 9 & 14 & 15 \\
$\mathrm{FH}+$ & 4 & 8 & 7 \\
Women & 7 & 12 & 10 \\
Age & $20.9(3.3)$ & $20.4(1.7)$ & $22.4(3.5)$ \\
BMI & $21.6(2.2)$ & $22.7(2.7)$ & $22.7(3.5)$ \\
BDI & $3.1(2.3)$ & $5.2(4.7)$ & $4.3(3.9)$ \\
\hline
\end{tabular}

\section{Design}

A placebo-controlled, double-blind design was used. During two experimental morning sessions, subjects visited the laboratory and affective processing was monitored before and after exposure to acute stress either following a tryptophan (TRP)-free (ATD) or TRP-containing (placebo or PLC) amino acid mixture. The order of presentation of both mixtures was counterbalanced within $\mathrm{FH}$ groups and the two experimental 
sessions were separated by two weeks. Female subjects were tested in the follicular phase of their menstrual cycle.

Plasma amino acid levels were obtained at baseline and 4.5 hours after administration of the amino acid mixture. Stress induction took place 5.5 hours after administration of the amino acid mixture and behavioral assessment was obtained at baseline, pre-stress and post-stress.

A reduction in brain 5-HT was accomplished by ATD through the use of a TRP-free collagen-protein (CP) amino acid drink (Blokland et al., 2004; Evers et al., 2005; Sambeth et al., 2008, Firk \& Markus, 2008).

\section{Stress exposure}

A mental arithmetic task, performed under noise stimulation, was used as an uncontrollable stress situation (Markus-Peters Arithmetic (MPA) Task). Subjects were given 8 successive 1-min trials during which they had to solve a specific number of multiple choice mental arithmetic problems (the criterion) under time-constraints, while at the same time continuous 75,80 or $85 \mathrm{~dB}$ industrial noise was presented to them through headphones. They were led to believe that the intensity of the noise depended on their performance; if they failed the criterion, noise intensity was chosen by the computer during the next trial; if they met the criterion, they could choose the intensity of the noise. In fact, the criterion was manipulated so that all subjects continued to fail on each trial. This task has been demonstrated to induce psychological and physiological stress and to be perceived as highly uncontrollable (Markus et al., 1998; Markus et al., 2000a; Markus et al., 2000b).

\section{Affective go/no-go}

A modified version of the affective go/no-go (AGNG) task described by Murphy et al. (1999) was used in the present study. In this task, happy and sad words are presented on the screen once by once for $300 \mathrm{~ms}$ followed by an inter-stimulus interval of 900 ms during which participants must make or withhold a button press response on the basis of the emotion of the word. The task comprised 2 practice blocks and 12 experimental blocks. Each block contained 18 words, 9 happy words and 9 sad words and subjects were instructed that they must respond either to the 
happy words or to the sad words before each block. Every two blocks, the targets and the distractors changed, words that were previously targets became distractors and words that were previously distractors became targets (SSHHSSHHSSHHSS or HHSSHHSSHHSSHH). Due to this arrangement shift blocks and non-shift blocks could be studied.

\section{Biochemical Analyses}

Genotyping. Buccal cell samples for measuring the 5-HTTLPR polymorphism of 5-HT transporter were obtained using sterile swabs (Omni Swabs, Whatman, 's Hertogenbosch, The Netherlands). Genomic DNA was isolated from buccal swabs using Olamp DNA Mini Kits from Qiagen for determination of the 5-HTTLPR genotype. Genotyping was performed using the polymerase chain reaction (PCR) protocol according to Glatz et al. (2003).

Plasma samples. Blood samples were collected in $5 \mathrm{ml}$ Vacutainer tubes containing sodium heparin for amino acids. The sodium-heparin tube was centrifuged at $5000 \mathrm{rpm}$ for $10 \mathrm{~min}$ at $4^{\circ} \mathrm{C}$. Subsequently, the supernatants were directly stored at $-80^{\circ} \mathrm{C}$ until analysis. Plasma amino acid analyses were conducted with HPLC, making use of a 2-3 $\mu \mathrm{m}$ Bischof Spherisorb ODS II column. The plasma TRP ratio was calculated by dividing the plasma TRP concentration by the sum of the other large neutral amino acids (LNAA's), i.e. valine, isoleucine, leucine, tyrosine and phenylalanine.

\section{Statistical analysis}

The main research questions were analyzed by means of repeated measures analyses of variance (ANOVA) by using the General Linear Model (GLM: SPSS 12.0 for Windows). To control for possible effects of order within genotype groups, all analyses were preliminary run with order as covariate but since this did not change our findings, order was left out of the final analyses. All statistics are evaluated at a two-tailed significance level of $5 \%$. 


\section{RESULTS}

\section{Biochemical measures}

Repeated measures analysis of variance with Genotype (s/s vs.s s/l vs. I/I) as between-subjects factor and Treatment (ATD vs. PLC) and Time $\left(t_{0}\right.$ vs. $t_{4.5}$ ) as within-subjects factors were carried out for total plasma TRP concentrations and for the TRP:LNAA ratio. For plasma TRP, a significant Treatment $X$ Time interaction was found $(F(1,35)=146.00, P<.001)$, reflecting a $62 \%$ decrease 4.5 hours after ATD and a $13 \%$ increase after PLC intake. For the TRP:LNAA ratio, a Treatment $X$ Time interaction was found $(F(1,35)=142.72, P<.001)$, reflecting a $65 \%$ decrease after ATD and a $8 \%$ increase after PLC. The Treatment $X$ Time $X$ genotype interaction was not significant for total plasma TRP concentrations $(F(2,35)=1.53$, $P=.23)$ and for the TRP:LNAA ratio $(F(2,35)<1$, ns).

\section{Affective go/no-go}

Reaction time, error and omission data were analyzed separately using 5-way repeated measures analysis of variance (ANOVA) with Genotype (ss vs. sl vs. II) as between-subjects factor and Treatment (ATD vs. PLC), Target valence (sad vs. happy), Shift (shift vs. non-shift) and Stress (pre-stress vs. post-stress) as within-subjects factors. In the interests of brevity, we focus only on the effects of Genotype or interactions with Genotype. Analysis revealed a significant interaction effect of Genotype $x$ Stress $x$ Shift on the number of omissions $(F(2,35)=4.78, P=.015)$, which is visualized in Figure 1. Individual comparisons showed significantly fewer omissions on shift blocks post-stress compared to pre-stress for participants with the $\mathrm{I} / \mathrm{I}$ genotype $(t(14)=2.64, P=.019)$. Individuals with an $s$ allele showed even more omissions post-stress compared to prestress on shift blocks, however this effect did not reach significance $(\mathrm{s} / \mathrm{s}$ genotype: $t(14)=1.69, P=.12, \mathrm{~s} /$ genotype: $t(14)=0.8, P=.44)$. 


\section{Effect of stress}

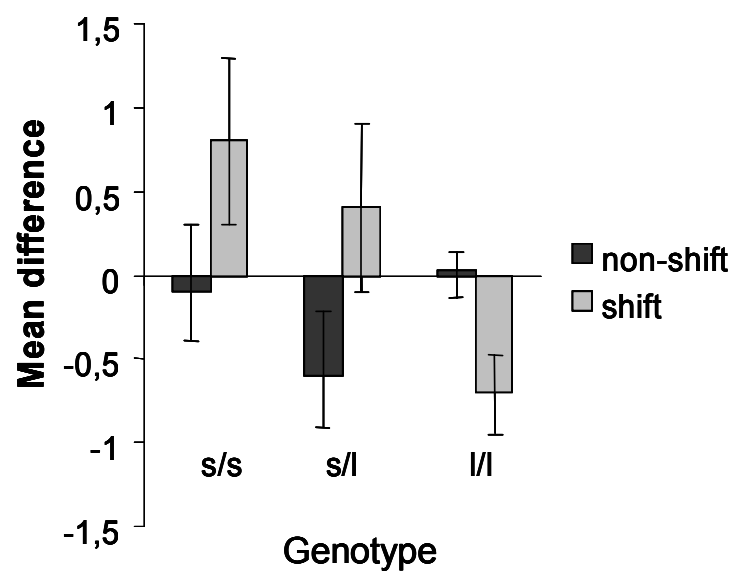

Figure 1. Mean difference in omissions pre-stress compared to post-stress for shift and non-shift trials for all genotypes.

\section{DISCUSSION}

The main goal of the present study was to test whether 5-HTTLPR genotype mediates the effects of ATD on affective processing following acute stress exposure. Contrary to our predictions that individuals with the s/s genotype would respond like depressive patients following ATD and stress induction, we did not find a negative affective bias (slowed responding to happy words) in individuals with the s/s genotype. In the present study, we found a genotype by stress by shift interaction indicating fewer omissions for targets on non-shift blocks post-stress compared to pre-stress for individuals with the I/I genotype. Thus, performance did not decline following acute stress but even improved for individuals with the I/I genotype. In accordance with our data, Rosier et al. (2006) did not find a negative affective bias either but reported that individuals with the I/I genotype made significantly more omissions than the s/s genotype. However, in this study, participants were not tested following acute stress exposure.

One limitation of the present study is that we did not analyze the recently identified additional variants of the 5-HTTLPR within the I allele 
(LG and LA Hu et al., 2005), which may have functional significance. Another limitation is that our genotype groups are relatively small, which may explain our negative findings on affective processing.

The genotype-related differential effects of acute stress exposure might be associated with higher tonic serotonergic neurotransmission in participants homozygous for the I allele. Therefore, subjects with the I/I genotype may have better stress coping strategies than s allele carriers. This is consistent with findings by Markus et al. (2000a; 2002) who reported that chronically-stressed students show improved stress coping and mood following 5-HT augmentation. The hypothesis that higher serotonergic neurotransmission in participants homozygous for the I allele is associated with better stress coping strategies may also explain the findings that individuals with the $1 / /$ genotype are less likely to develop depression following stressful life events. However, this tentative explanation merits further research 



\section{CHAPTER 4}

Differential effects of 5-HTTLPR genotypes on mood, memory and attention bias following acute tryptophan depletion and stress exposure

Based on:

Firk, C., Markus, C.R. (in press). Differential effects of 5-HTTLPR genotypes on mood, memory and attention bias following acute tryptophan depletion and stress exposure. Psychopharmacology. 


\section{ABSTRACT}

\section{Rationale}

Polymorphisms of the serotonin transporter gene (5-HTTLPR) may be associated with increased vulnerability to acute tryptophan depletion (ATD) and depression vulnerability especially following stressful life events.

\section{Objectives}

The aim of the present study was to investigate the effects of ATD in subjects with different 5-HTTLPR profiles before and after stress exposure on affective and cognitive-attentional changes.

\section{Methods}

18 subjects with homozygotic short alleles $\left(S^{\prime} / S^{\prime}\right)$ and 17 subjects with homozygotic long alleles ( $\left.L^{\prime} / L^{\prime}\right)$ of the 5-HTTLPR participated in a double-blind, placebo-controlled, crossover design to measure the effects of ATD on mood, memory and attention before and after acute stress exposure.

Results

ATD lowered mood in all subjects independent of genotype. In $S^{\prime} / S^{\prime}$ genotypes mild acute stress increased depressive mood and in $L^{\prime} / L^{\prime}$ genotypes increased feelings of vigor. Furthermore, $S^{\prime} / S^{\prime}$ genotypes differed from $L^{\prime} / L^{\prime}$ genotypes on measures of attention independent of treatment, and memory following ATD.

Conclusion

Polymorphisms of the 5-HTTLPR differentially affects responses to mild stress and ATD, suggesting greater vulnerability of $S^{\prime} / S^{\prime}$ carriers to serotonergic manipulations and supporting increased depression vulnerability. 


\section{INTRODUCTION}

Dysregulation of the serotonergic system has been implicated in the pathophysiology of depression (Maes \& Meltzer, 1995). This is based on studies reporting lower plasma availability of the serotonin (5-HT) precursor tryptophan (TRP) for uptake into the brain, reduced cerebrospinal fluid (CSF) concentration of the 5-HT metabolite 5-hydroxyindoleacetic (5-HIAA) and decreased platelet 5-HT uptake in depressed patients, suggesting diminished brain 5-HT uptake and metabolism (Maes \& Meltzer, 1995; Neumeister et al., 2004c). The level of 5-HT in the synaptic cleft is mainly regulated by the $5-\mathrm{HT}$ transporter (5-HTT), which is targeted by most antidepressants (Suhara et al., 2003; Meyer et al., 2004; Voineskos et al., 2007). Interestingly, the human 5-HTT gene, located on chromosome 17q11.1-q12 (Lesch et al., 1994), contains a 5-HTT-linked polymorphic region (5-HTTLPR) with two functional variants: the short (s) form and the long (I) form (Heils et al., 1996; Greenberg et al., 1999). The $\mathrm{s}$ form of this variant is less active, resulting in reduced transcriptional efficiency of the 5-HTT gene, decreased 5-HTT expression and reduced 5-HT uptake relative to the I form (Heils et al., 1996; Greenberg et al., 1999). The s allele has been associated with increased risk of depression (Collier et al., 1996; Joiner et al., 2003; Gonda et al., 2005; Gonda et al., 2006), poorer responses to the antidepressant effects of SSRIs (Smeraldi et al., 1998; Serretti et al., 2007), increased vulnerability to tryptophan depletion (Neumeister et al., 2002; Roiser et al., 2006) and abnormal emotional processing (Hariri et al., 2002; Marsh et al., 2006).

A direct association between 5-HTTLPR genotypes and depressionrelated phenotypes appears to be relatively weak (Lotrich \& Pollock, 2004). Roiser (2005) only found abnormal emotional processing in 3,4 methylenedioxymethamphetamine (MDMA, or Ecstasy) users carrying the s allele. MDMA prevents uptake of 5-HT and stimulates the release of $5-\mathrm{HT}$, causing long-term changes to the $5-\mathrm{HT}$ system. This suggests that $\mathrm{s}$ allele carriers may only have a predisposition to serotonergic vulnerability that may be unmasked through challenges to the serotonergic system. This is also in line with contemporary theoretical models of gene involvement in depression that postulate that genetic vulnerability does not affect depression directly; rather, 5-HTTLPR may enhance susceptibility for depression particularly in the face of severe life events or stress- 
ful experiences, which may also challenge the brain serotonergic system (Firk \& Markus, 2007). Hence, depression is often preceded by stress (Van Praag, 2004), and epidemiological studies have already demonstrated a positive association between the s allele of the 5-HTTLPR and a depressive reaction to stressful life events (e.g. Caspi et al., 2003; for a recent review see Uher \& McGuffin, 2008). This is in line with the hypothesis that stress may cause depression due to complex interactions between the neuroendocrine stress system and the serotonergic system, particularly in individuals with a biological (genetic) vulnerability related to their serotonergic system (Van Praag, 2004; Firk \& Markus, 2007). Although explorations of the involvement of interactions between serotonergic vulnerability in s allele 5-HTTLPR and stress in the onset of affective symptoms is a major challenge in biological psychiatry today, this interaction has not yet been experimentally investigated.

On the other hand, the relatively small direct association between 5HTTLPR genotypes and depression related phenotypes may also be explained by recently identified functional variants within the I allele $\left(L_{A}\right.$ and $L_{G}$ ) that may underestimate the effect of 5-HTTLPR. This is because the $\mathrm{L}_{\mathrm{G}}$ allele has a low rate of $5-\mathrm{HTT}$ expression comparable to the $\mathrm{s}$ allele and is relatively common in Caucasian and African American populations (Hu et al., 2006; Zalsman et al., 2006).

One accepted method to study behavioral and cognitive effects of transiently reduced $5-\mathrm{HT}$ availability in humans is the acute tryptophan depletion (ATD) strategy (Young et al., 1985; for a review see Fusar-Poli et al., 2006). In this procedure, brain 5-HT is reduced through depletion of plasma tryptophan (TRP, a precursor of 5-HT) availability for uptake into the brain by intake of a TRP-free amino acid mixture that contains all of the essential amino acids except for TRP. This raises incorporation of TRP into protein synthesis and reduces TRP relative to the sum of the other Large Neutral Amino Acids (LNAA's) with which TRP competes for uptake into the brain (i.e. Fernstrom \& Wurtman, 1971; Gessa et al., 1974; Moja et al., 1991; Maes \& Meltzer, 1995). Evidence for reduced 5HT neurotransmission after ATD comes from studies measuring 5-HT metabolites (5-HIAA) in cerebrospinal fluid (Carpenter et al., 1998; Williams et al., 1999). In addition, there is also plenty of evidence showing that the ATD procedure is the most suitable method to study brain serotomergic vulnerability. For instance, mood lowering effects of tryptophan depletion are clearly found in depressive patients in remission (Delgado 
et al., 1990; Delgado et al., 1999; Moreno et al., 2000; Ruhe et al., 2007), whereas in healthy individuals such mood lowering effects seem to depend on the 5-HTTLPR genotype (Neumeister et al., 2002; Neumeister et al., 2006; Roiser et al., 2007; Walderhaug et al., 2007) and family history of depression (Benkelfat et al., 1994; Klaassen et al., 1999; Neumeister et al., 2002). Individuals with a positive family history of depression are more vulnerable to the mood lowering effects of ATD than individuals without a family history of depression, suggesting an (innate) vulnerability of the serotonergic system in relatives of depressive patients (Benkelfat et al., 1994; Klaassen et al., 1999; Neumeister et al., 2002). However, the effects of ATD on mood in individuals with different 5-HTTLPR profiles are contradictory. Neumeister et al. (2002) reported mood lowering in healthy s allele carriers, in particular in those with a positive family history of depression. Walderhaug et al. (2007) found mood lowering following ATD only in women homozygous for the I and s allele, whereas other studies did not find mood lowering effects at all (Marsh et al., 2006; Roiser et al., 2007). Therefore, we suggest that the possession of the $s$ allele, which is very common in the population, may not alone increase risk, but may induce enhanced susceptibility to ATD only in the context of stress.

The aim of the present study was to investigate the effects of ATD in subjects with different 5-HTTLPR profiles before and after stress exposure on affective and cognitive-attentional changes that are thought to be critical in the etiology and maintenance of depression. A selective attention and memory bias for negative information is the most commonly recognized characteristic of depression and depression vulnerability (e.g. Bradley et al., 1995; Mogg \& Bradley, 2005; Rinck \& Becker, 2005). For instance, depressive patients show memory biases for negative information in explicit memory free recall tasks (e.g. Bradley et al. 1995; Lim and Kim 2005) and slowed responses to happy words compared to sad words during affective go/no-go tasks (Murphy et al., 1999; Erickson et al., 2005). In addition, comparable attentional biases are also found in healthy individuals following ATD, further suggesting that serotonergic factors are particularly involved (Murphy et al., 2002). Based on the literature and the data reviewed above, we hypothesize that the 5HTTLPR genotype mediates ATD- and stress-induced changes in mood and cognitive-behavioral indications of affective biases. To our knowledge, this is the first time the depressogenic effects of reducing brain 5- 
HT by ATD are investigated in 5-HT-vulnerable s allele 5-HTTLPR carriers before and after stress exposure.

\section{METHODS}

\section{Participants}

Undergraduate students at Maastricht University ( $N=200)$ completed a screening questionnaire concerning general information (health, smoking and drinking habits, caffeine consumption, use of psychoactive drugs, past and present treatment with medication, weight and height, eating habits, and more). Furthermore, the Beck Depression Inventory (BDI) (Beck et al., 1961) was completed to verify the absence of depressive symptomatology.

Participants were excluded if they reported chronic and current illness; history of psychiatric or medical illness; family history of depression; medication use; metabolic-, hormonal- or intestinal diseases; irregular diets or deviant eating habits; drug use; or excessive (more than 10 units per week) alcohol use. Participants' health was checked with standardized medical questionnaires that were evaluated by a trained doctoral-level psychologist under the supervision of a medical doctor. We acknowledge that, although participants reported not having any medical illness, a medical examination might have been a likely additional method to further ensure participants' physical health. Participants included in the study revealed normal body-mass indexes (BMl in $\mathrm{kg} / \mathrm{m}^{2}$ between 19-26), were non-smokers and were requested not to use alcohol before or during the study.

From the subjects showing interest in taking part in the experiment and fulfilling to the in- and exclusion criteria, 86 participants were invited to attend an initial buccal sample extraction session. During this session, buccal cell samples were taken via swabs to genotype all individuals for 5-HTTLPR. Participants were compensated $(€ 5)$ for participation in this buccal sample extraction phase of the study. All subjects had to give informed consent to participate during this buccal DNA extraction session.

Participants with the $S / S, S / L_{G}$, or $L_{G} / L_{G}$ genotype classified as $S^{\prime} / S^{\prime}$ and participants with the $L_{A} / L_{A}$ genotype classified as $L^{\prime} / L^{\prime}$ were selected 
for the experiment and received oral and written information about the study. Eighteen subjects with the $\mathrm{S}^{\prime} / \mathrm{S}^{\prime}$ genotype and 17 subjects with the $L^{\prime} / L^{\prime}$ genotype completed the experiment. The $S^{\prime} / S^{\prime}$ group and the $L^{\prime} / L^{\prime}$ group did not differ with respect to sex, age, BMI or BDI scores (all $p>$.05). Demographic characteristics are presented in Table 1.

The study was approved by the Medical Ethics Committee of the Academic Hospital Maastricht (CTCM azM; Maastricht; The Netherlands) and the procedures followed were in accordance with the Helsinki Declaration of 1975 as revised in 1983. All subjects gave their informed consent to participate in the experiment and were paid $150 €$ for participation.

Table 1. Demographic characteristics. Values represent mean (SD).

\begin{tabular}{lll}
\hline & Genotype & \\
\cline { 2 - 3 } & $\mathrm{S}^{\prime} / \mathrm{S}^{\prime}$ & $\mathrm{L}^{\prime} / \mathrm{L}^{\prime}$ \\
\hline Women & 17 & 18 \\
Men & 2 & 2 \\
Age & $19.6(1.7)$ & $19.5(1.8)$ \\
BMI & $22.0(1.8)$ & $21.4(2.2)$ \\
BDI & $2.7(2.4)$ & $2.8(2.6)$ \\
\hline
\end{tabular}

\section{Design}

A placebo-controlled, double-blind, crossover design was used. During two experimental sessions, subjects were monitored for affective processing before and after acute stress exposure either following intake of a TRP-free (TRP-) or a TRP-containing balanced (TRP+) amino acid mixture. The order of presentation of the TRP- and TRP+ condition was counterbalanced within groups and both experimental sessions were separated by at least one week. Before and $4.5 \mathrm{~h}$ after intake of the amino acid mixtures, blood samples were taken to measure the effect of ATD on plasma amino acid concentrations and the TRP:LNAA ratio. Female subjects were tested in the follicular phase of their menstrual cycle (days 4-10) or when actually taking oral contraceptives. 


\section{Procedure}

All participants were instructed to fast overnight; only water or tea without sugar was permitted. On each experimental morning, participants arrived for testing at 08:30 a.m., 09:00 a.m., 09:30 a.m. and 10:00 a.m., respectively, and a first blood sample was taken to obtain baseline plasma TRP and LNAA levels. A second blood sample was drawn $4.5 \mathrm{~h}$ after intake of the amino acid mixture (TRP- or TRP+) to assess changes in total TRP concentrations and TRP:LNAA ratios. At baseline and $4.5 \mathrm{~h}$ following intake of the amino acid mixture, subjects completed a survey measuring vegetative side effects. Five hours after administration of the amino acid mixture, participants' mood was assessed with the Profile of Mood States (POMS Wald \& Mellenbergh, 1990) and the Positive and Negative Affect Scale (PANAS, Watson et al., 1988). Mood assessment was followed by the affective go/no-go task (to measure attentional biases) and the emotional related free recall task (to measure memory biases). Then, participants were exposed to an uncontrollable stress task (MPA task), again followed by mood assessment and the affective go/nogo task and the emotional related free recall task. Further, two pre-stress salivary cortisol samples were taken (at $4.5 \mathrm{~h}\left(\mathrm{t}_{-30}\right)$ and immediately before the MPA task $\left.\left(t_{0}\right)\right)$ and two post-stress cortisol samples were taken (immediately after the MPA task $\left(t_{+20}\right)$ and 10 minutes after finishing the MPA task $\left.\left(t_{+30}\right)\right)$ to measure stress-related activation of the HPA axis. Between intake of the amino acid mixture and exposure to laboratory tasks, the subjects were able to study or to read magazines in a separate private room. They had free access to water and decaffeinated tea. Two hours after administration of the amino acid mixture, they received a standardized protein-poor lunch as previously used in ATD studies (Riedel et al., 1999; Sobczak et al., 2002a; Sobczak et al., 2002b). At the end of each test day, subjects received a high protein snack and bananas, which are natural sources of L-tryptophan to facilitate a quick recovery from possible negative effects of ATD.

\section{Acute tryptophan depletion}

A reduction in brain 5-HT was accomplished by acute tryptophan depletion (ATD) through the use of a TRP-free collagen-protein (CP) amino acid drink (Blokland et al., 2004; Evers et al., 2005; Sambeth et al., 
2008). To obtain a drinkable mixture, $100 \mathrm{~g}$ of the protein powder was mixed with $200 \mathrm{ml}$ of tap water. During the TRP+ condition the intake of the mixture was directly followed by ingestion of four capsules containing $1.2 \mathrm{~g}$ L-TRP (Sigma, Zwijndrecht; The Netherlands), whereas during the TRP- condition intake was directly followed by ingestion of four capsules containing lactose (all capsules were taken with a little water). See Table 2 for the amino acid composition of the different conditions (Evers et al., 2005).

Table 2. Composition (grams) of the gelatin-based protein (all values are $\mathrm{g}$ per $100 \mathrm{~g}$ of each mixture)

\begin{tabular}{lll}
\hline & TRP- & TRP+ \\
\hline Phenylalanine & 1.9 & 1.9 \\
Tyrosine & 0.4 & 0.4 \\
Valine & 2.1 & 2.1 \\
Leucine & 3 & 3 \\
Isoleucine & 1.4 & 1.4 \\
Tryptophan & 0.1 & 1.3 \\
Serine & 3.1 & 3.1 \\
Glycine & 22.5 & 22.5 \\
Histidine & 0.5 & 0.5 \\
Arginine & 8.8 & 8.8 \\
Threonine & 1.1 & 1.1 \\
Alanine & 9.3 & 9.3 \\
Proline & 13.3 & 13.3 \\
Methionine & 0.6 & 0.6 \\
Cystein & 0.2 & 0.2 \\
Lysine & 3.6 & 3.6 \\
Hydroxyproline & 12.1 & 12.1 \\
Hydroxylysine & 1.4 & 1.4 \\
Aspartic acid + asparagines & 9.3 & 9.3 \\
Glutamic acid + glutamine & 5.2 & 5.2 \\
\hline
\end{tabular}

This ATD method differs from the classic methodology by including a gelatin-based hydrolyzed collagen-protein (CP) that contains the entire range of amino acids (except for L-TRP) in the form of peptides. After administration, these peptides are decomposed into amino acids and the mechanism of depletion is identical to the classical ATD method. 


\section{Stress exposure}

A mental arithmetic task, performed under noise stimulation, was used as an uncontrollable stress situation (Markus-Peters Arithmetic (MPA) Task). Subjects were given 18 successive 1-min trials during which they had to solve a specific number of multiple choice mental arithmetic problems (the criterion) under time-constraints, while at the same time continuous 75,80 or $85 \mathrm{~dB}$ industrial noise was presented to them through headphones. They were led to believe that the intensity of the noise depended on their performance; if they failed the criterion, the noise intensity was chosen by the computer during the next trial; if they met the criterion, they could choose the intensity of the noise. In fact, the criterion was manipulated so that all subjects continued to fail on each trial. This task has been demonstrated to induce psychological and physiological stress and to be perceived as highly uncontrollable (Markus et al., 1998; Peters et al., 1998; Markus et al., 2000a; Markus et al., 2000b). In the present study, an adapted version of the MPA was used during which subjects had to perform under audience observation. Furthermore, subjects were told that a video analysis of their performance was conducted to analyze their nonverbal behavior.

\section{Subjective mood ratings}

Profile of Mood States (POMS). Changes in mood were measured using the Dutch shortened version of the POMS questionnaire (Wald \& Mellenbergh, 1990), offered at the computer-screen as a VAS scale ranging from 'strongly disagree' to 'strongly agree'. The POMS comprises five different subscales for mood; ranging from Anger, Depression, Fatigue and Tension that refer to a negative mood state, to Vigor concerning a positive mood.

Positive and Negative Affect Scale (PANAS). The PANAS (Watson et al., 1988) consists of two 10-item mood scales, a positive mood scale and a negative mood scale. The 20 items are randomly presented on a computer screen and participants have to respond on a scale ranging from 1 (totally disagree) to 5 (totally agree). 


\section{Emotional related free recall}

To test emotionally related immediate and delayed free recall, an adapted version of the 30 Word Learning Test ( 30 WLT) was used (e.g. Sobczak et al., 2002b; Smeets et al., 2006). The 30 WLT is an adapted version of the Rey Auditory Verbal Learning Task (Lezak, 2004). In this test, participants are presented with a list of 30 words ( 10 negative, 10 positive, and 10 neutral words matched for frequency, word length and emotional valence, chosen from Hermans and De Houwer's (1994) list of Dutch words that have been rated for familiarity and affectivity) in random order. Each word was presented on the computer monitor for $3 \mathrm{~s}$ with an inter-stimulus interval of $1 \mathrm{~s}$. The participants had to rate how relevant each word was to their personal concerns on a scale ranging from 1 (not at all relevant) to 5 (extremely relevant). Following the encoding task, the participants were given 2 min to complete an immediate written recall task. A delayed recall took place after 30 minutes. Four comparable lists (matched for frequency, word length and emotional valence) were used and presentation order was counterbalanced across participants.

\section{Affective go/no-go}

A modified version of the affective go/no-go (AGNG) task described by Murphy et al. (1999) was used in the present study. In this task, happy and sad words are presented on the screen one-by-one for $300 \mathrm{~ms}$ followed by an inter-stimulus interval of $900 \mathrm{~ms}$ during which participants must make or withhold a button press response on the basis of the emotion of the word. The task comprises 2 practice blocks and 12 experimental blocks. Each block contains 18 words (9 happy words and 9 sad words) and subjects are instructed that they must respond either to the happy words or to the sad words before each block. Every two blocks, the targets and the distractors change, words that were previously targets become distractors and words that were previously distractors become targets (SSHHSSHHSSHHSS or HHSSHHSSHHSSHH). Due to this arrangement, shift blocks and non-shift blocks can be studied. 


\section{Vegetative side effects}

In order to measure possible side effects of the amino acid mixtures, a list (5-point scales) of vegetative side effects was completed before and $4.5 \mathrm{~h}$ after intake. The list contained the following items: feeling cold, feeling hot, dizziness, perspiration, blurred vision, nausea, palpitations, dry mouth and abdominal complaints.

\section{Biochemical measurements}

Plasma samples. Blood samples were collected in $5 \mathrm{ml}$ Vacutainer tubes containing sodium heparin for amino acid measurements. The sodium-heparin tube was centrifuged at $5000 \mathrm{rpm}$ for $10 \mathrm{~min}$ at $4^{\circ} \mathrm{C}$. Subsequently, the supernatants were directly stored at $-80^{\circ} \mathrm{C}$ until analysis. Plasma amino acid analyses were conducted with HPLC, making use of a 2-3 $\mu \mathrm{m}$ Bischof Spherisorb ODS II column. The plasma TRP ratio was calculated by dividing the plasma TRP concentration by the sum of the other large neutral amino acids; i.e., valine, isoleucine, leucine, tyrosine and phenylalanine.

Salivary cortisol. Cortisol samples were obtained by using the Salivette sampling device (Sarstedt@, Etten-Leur, The Netherlands). With this procedure, saliva was collected in small cotton swabs and stored ($25^{\circ}$ Celsius) immediately upon collection until centrifugation. Saliva samples were centrifuged at $2650 \mathrm{~g}_{\max }$ for three minutes at $20^{\circ} \mathrm{C}$. Salivary free cortisol levels were determined in duplicate by direct radioimmunoassay (RIA; University of Liège, Belgium), including a competition reaction between ${ }^{125}$ iodohistamine-cortisol and anti-cortisol serum made against the 3-CMO-BSA conjugate. After incubation of $100 \mu \mathrm{l}$ of saliva at $4^{\circ}$ Celsius overnight, separation of free and antibody-bound ${ }^{125}$ iodohistamine-cortisol was performed via a conventional 'second antibody' method. In order to reduce sources of variability, all samples were analyzed in the same assay.

Buccal cells for polymorphism assessment. Buccal cell samples for measuring triallelic variants of the 5-HTT-linked polymorphic region (5HTTLPR) were obtained using sterile swabs (Omni Swabs, Whatman's Hertogenbosch, The Netherlands). Genomic DNA was isolated from buccal swabs using OlAamp DNA Mini Kits from Qiagen (Westburg, Leusden, The Netherlands) for determination of the 5-HTTLPR genotype. 
Briefly, genotyping was performed using the polymerase chain reaction (PCR) protocol according to Wendland et al. (2006) with the oligonucleotide primers 5'-TCCTCCGCTTTGGCGCCTCTTCC-3' and 5'TGGGGGTTGCAGGGGAGATCCTG-3'. Triallelic variants were reclassified into a biallelic model as follows: $S / S, S / L_{G}$ and $L_{G} / L_{G}$ were classified as $S^{\prime} / S^{\prime}$ and $L_{A} / L_{A}$ as $L^{\prime} / L^{\prime}$ (Neumeister et al., 2006; Zalsman et al., 2006; Walderhaug et al., 2007).

\section{Statistical analysis}

The main research questions were analyzed by means of repeated measures multivariate and univariate analyses of variance (MANOVA and ANOVA) by using the General Linear Model (GLM: SPSS 12.0 for Windows) with one between-subjects factor Genotype ( $S^{\prime} / S^{\prime}$ vs. $\left.L^{\prime} / L^{\prime}\right)$ and two within-subjects factors Treatment (TRP+ vs. TRP-) and Stress (prestress vs. post-stress) or Time ( $t_{0}$ vs. $t_{4.5}$ or $\left.t_{5}\right)$ (cortisol analysis: Time $\left(t_{-30}\right.$ vs. $t_{0}$ vs. $t_{+20}$ vs. $\left.t_{+30}\right)$ ) on the several dependent measures. Order was included as centered covariate. For the subjective mood ratings, we conducted two repeated measures ANOVAs with Genotype ( $S^{\prime} / S^{\prime}$ vs. $\left.L^{\prime} / L^{\prime}\right)$ as the between-subjects factor and Treatment (TRP+ vs. TRP-) as the within-subjects factors for the subjective mood ratings. To investigate the effects of treatment and the effects of stress separately, we included Time ( $t_{0}$ vs. $\left.t_{5}\right)$ in the first analysis and Stress (pre-stress vs. post-stress) in the second analysis as within-subjects factor. For the AGNG task, Valence (positive vs. negative) and Shift (shift blocks vs. non-shift blocks) were included as within-subjects factors. For the emotional related free recall, Valence (negative vs. neutral vs. positive) was included as a withinsubjects variable. Greenhouse-Geisser corrected $P$ values are reported when the sphericity assumption is not met. All statistics are evaluated at a two-tailed significance level of $5 \%$. Post hoc analyses for significant interactions were assessed by paired and unpaired t-tests to aid interpretation; corrections for multiple comparisons were not carried out. 


\section{RESULTS}

\section{Plasma amino acids (Total TRP and TRP:LNAA ratio)}

For TRP concentrations a significant Treatment $X$ Time interaction was found $[F(1,32)=224.87, p<.001]$, reflecting a decrease from $t_{0}$ to $t_{4.5}$ by $79 \%$ after TRP- and an increase from $t_{0}$ to $t_{4.5}$ by $68 \%$ after TRP+ administration. Analysis of the plasma TRP:LNAA ratio revealed a significant Treatment $X$ Time interaction $[F(1,32)=269.59, p<.001]$ with a $77 \%$ decline in plasma TRP:LNAA after TRP- and an increase from $t_{0}$ to $t_{4.5}$ by $62 \%$ after TRP+ (see Figure 1). No other main or interaction effects were found including Genotype.

Total TRP

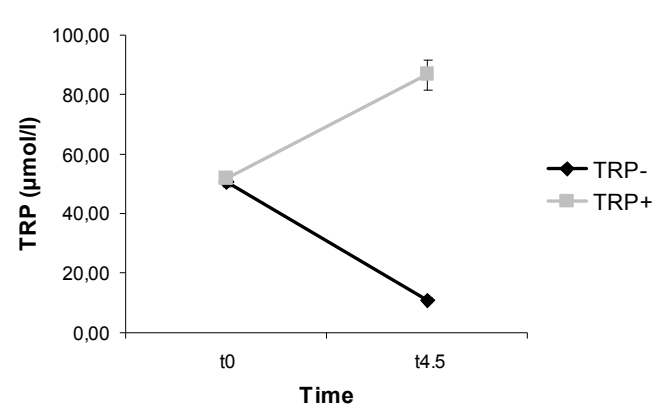

TRP/LNAA ratio

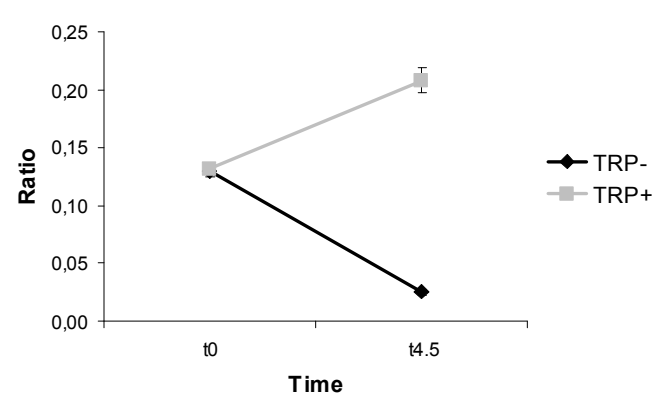

Figure 1. Plasma total TRP concentrations and TRP:LNAA ratio for the TRP- and TRP+ condition. For TRP concentrations, we found a significant decrease from $t_{0}$ to $t_{4.5}$ after TRP$[t(34)=24.56, p<.001]$ and a significant increase from $t_{0}$ to $t_{4.5}$ by $68 \%$ after TRP+ $[t(34)=7.58, p<.001]$. For the TRP:LNAA ratio, we found a significant decrease from $t_{0}$ to $t_{4.5}$ after TRP- $[t(34)=23.07, p<.001]$ and a significant increase from $t_{0}$ to $t_{4.5}$ by $68 \%$ after $\mathrm{TRP}+[t(34)=7.63, p<.001]$. 


\section{Cortisol}

Repeated measures analysis of variance only revealed a main effect of Time $[F(3,30)=4.02, p=.016]$ showing a slight but significant decline in cortisol concentrations $t_{+20}$ to $t_{+30}$ (from $2.4 \pm 0.2$ to $2.2 \pm 0.2 \mathrm{nmol} / \mathrm{L}$ ).

\section{Vegetative side effects}

No significant main or interaction effects were found.

\section{Subjective mood ratings}

Data for the subjective mood rating scales are presented in Table 3.

$P O M S$. In the first analysis, multivariate analysis revealed a main effect of Time $[F(5,28)=12.52, p<.001]$ with higher anger and fatigue scores and lower tension scores at $t_{5}$. Further, univariate analysis of the POMS revealed a Treatment $X$ Time interaction of the vigor subscale $[F(1,32)=7.28, p=.011]$ and fatigue subscale $[F(1,32)=5.35, p=.027]$ reflecting a stronger decrease in vigor ratings following TRP- than TRP+ $[t(34)=2.74, p=.01]$ and a stronger increase in fatigue ratings following TRP- than TRP $+[t(34)=2.32, p=.026]$ (see Figure 2).

In the second analysis, multivariate analysis showed a main effect of Stress $[F(5,28)=3.81, p=.009]$ and a significant Stress $X$ Genotype interaction $[F(5,28)=3.96, p=.008]$ (see Figure 3). Further, univariate analysis showed that the main effect of Stress originated from the subscale anger $[F(1,32)=12.39, p=.001]$ and fatigue $[F(1,32)=4.51, p=.041]$, reflecting an increase in anger post-stress compared to pre-stress and a decrease in fatigue post-stress compared to pre-stress. The Stress $X$ Genotype interaction originated from the depression subscale $[F(1,32)=8.24, p=$ $.007]$ and the vigor subscale $[F(1,32)=13.12, p=.001]$. This interaction was explored with a one-way multivariate analysis including the vigor and depression subscale for each genotype, indicating an increase in depressive mood after stress in the $S^{\prime} / S^{\prime}$ group $[F(1,16)=5.38, p=.034]$ only and an increase in vigor after stress in the $L^{\prime} / L^{\prime}$ group $[F(1,15)=12.61, p=.003]$ only (Figure 3 ).

PANAS. In the first analysis, we found a Treatment $X$ Time interaction $[F(1,32)=6.94, p=.013]$ on the positive scale, indicating a decrease in 
positive mood regardless of genotype following TRP- $[t(34)=2.57, p=$ $.015]$ but not following TRP $+[t(34)<1$, ns] (see figure 2$)$. No effects were found for the negative scale of the PANAS.

In the second analysis, only a significant effect of Stress $[F(1,32)=4.28, p=.047]$ was found on the negative scale, reflecting higher negative mood scores post-stress compared to pre-stress regardless of treatment and genotype.
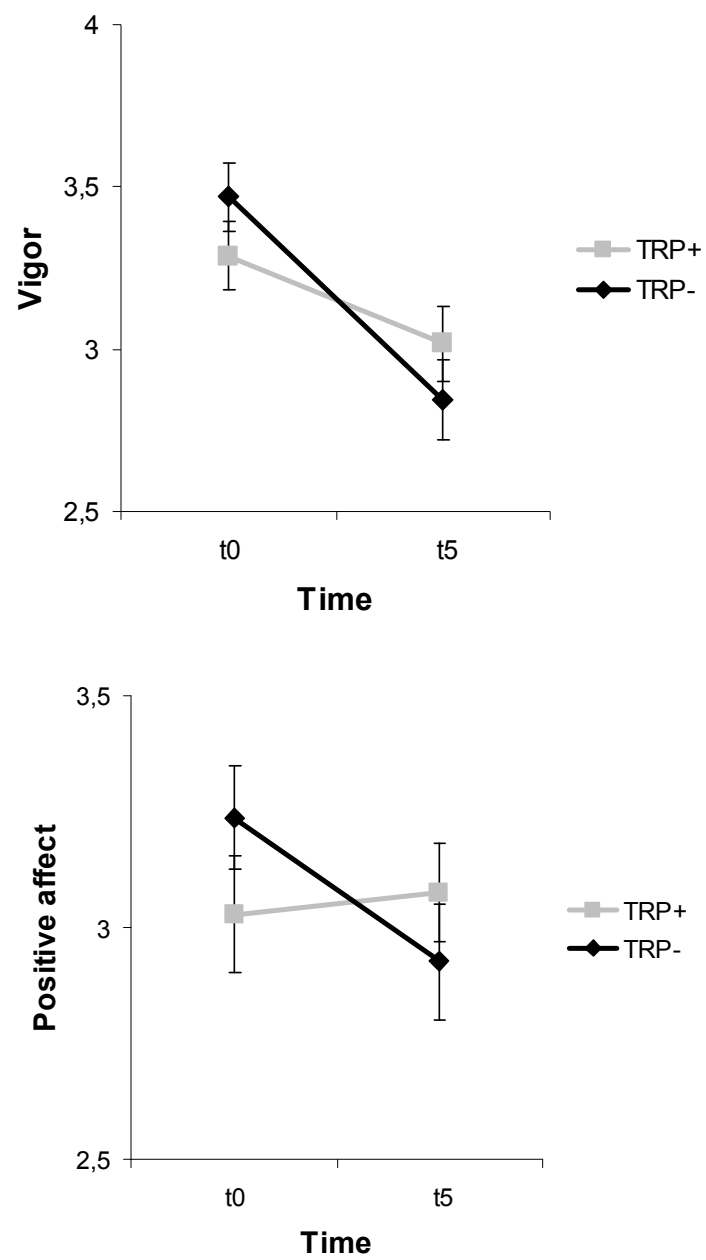

Figure 2. Mean (SE) vigor (POMS) and positive affect (PANAS) ratings showing a significant stronger decrease in vigor ratings following TRP- than TRP $+[t(34)=2.74, p=.01]$ and a significant decrease in positive affect ratings following TRP- but not TRP $[t(34)=2.57, p=$ .015]. 

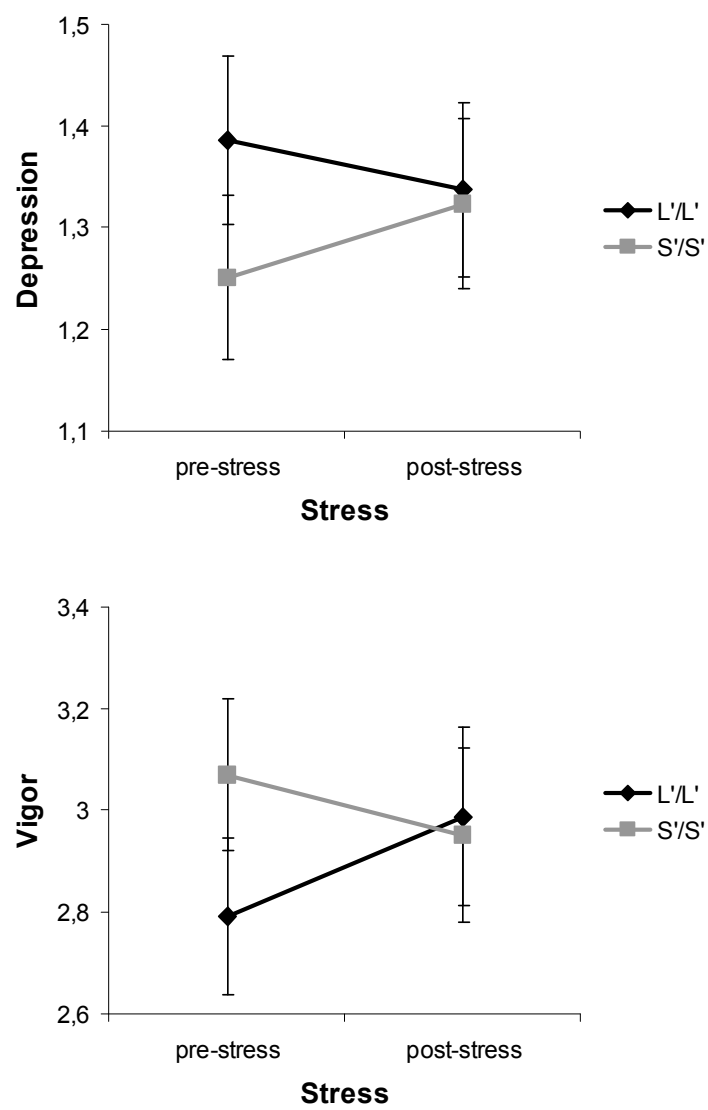

Figure 3. Mean (SE) depression and vigor ratings pre-stress and post-stress for both genotypes collapsed over treatment showing a significant increase in vigor post-stress for the $L^{\prime} / L^{\prime}$ genotype $[F(1,15)=12.61, p=.003]$ and a significant increase in depressive mood for the $S^{\prime} / S^{\prime}$ genotype $[F(1,16)=5.38, p=.034]$. 
Table 3. Subjective mood ratings. Values represent mean (SD).

\begin{tabular}{|c|c|c|c|c|c|c|c|}
\hline \multirow[b]{3}{*}{ Test } & \multirow[b]{3}{*}{ Measure } & \multicolumn{6}{|c|}{ 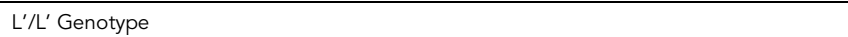 } \\
\hline & & \multicolumn{3}{|l|}{ TRP+ } & \multicolumn{3}{|l|}{ TRP- } \\
\hline & & Baseline & Pre-stress & Post-stress & Baseline & Pre-stress & Post-stress \\
\hline \multicolumn{8}{|c|}{ PANAS } \\
\hline & Positive & $2.9(0.8)$ & $3.2(0.7)$ & $3.1(0.8)$ & $3.3(0.8)$ & $3.0(0.8)$ & $3.0(0.9)$ \\
\hline & Negative & $1.2(0.2)$ & $1.2(0.2)$ & $1.2(0.2)$ & $1.3(0.3)$ & $1.2(0.3)$ & $1.3(0.4)$ \\
\hline \multicolumn{8}{|c|}{ POMS } \\
\hline & Anger & $1.3(0.2)$ & $1.4(0.4)$ & $1.5(0.6)$ & $1.3(0.2)$ & $1.5(0.4)$ & $1.5(0.4)$ \\
\hline & Depression & $1.4(0.3)$ & $1.4(0.3)$ & $1.3(0.2)$ & $1.3(0.2)$ & $1.4(0.4)$ & $1.4(0.5)$ \\
\hline & Fatigue & $1.7(0.5)$ & $2.0(0.7)$ & $1.8(0.6)$ & $1.5(0.5)$ & $2.3(0.9)$ & $2.1(0.9)$ \\
\hline & Tension & $1.5(0.4)$ & $1.4(0.3)$ & $1.4(0.3)$ & $1.7(0.5)$ & $1.4(0.4)$ & $1.4(0.4)$ \\
\hline & Vigor & $3.1(0.7)$ & $2.9(0.7)$ & $2.9(0.8)$ & $3.3(0.6)$ & $2.7(0.7)$ & $3.0(0.7)$ \\
\hline \multirow[b]{3}{*}{ Test } & \multirow[b]{3}{*}{ Measure } & \multicolumn{3}{|c|}{$\mathrm{S}^{\prime} / \mathrm{S}^{\prime}$ Genotype } & & & \\
\hline & & \multicolumn{3}{|l|}{ TRP+ } & \multicolumn{3}{|l|}{ TRP- } \\
\hline & & Baseline & Pre-stress & Post-stress & Baseline & Pre-stress & Post-stress \\
\hline \multicolumn{8}{|c|}{ PANAS } \\
\hline & Positive & $3.1(0.7)$ & $3.0(0.6)$ & $2.8(0.7)$ & $3.2(0.6)$ & $2.8(0.8)$ & $2.7(0.8)$ \\
\hline & Negative & $1.1(0.2)$ & $1.1(0.2)$ & $1.2(0.3)$ & $1.2(0.2)$ & $1.2(0.3)$ & $1.3(0.3)$ \\
\hline \multicolumn{8}{|c|}{ POMS } \\
\hline & Anger & $1.3(0.2)$ & $1.3(0.4)$ & $1.5(0.6)$ & $1.3(0.3)$ & $1.3(0.3)$ & $1.6(0.7)$ \\
\hline & Depression & $1.2(0.2)$ & $1.2(0.2)$ & $1.3(0.5)$ & $1.2(0.2)$ & $1.2(0.3)$ & $1.3(0.4)$ \\
\hline & Fatigue & $1.5(0.4)$ & $1.9(0.9)$ & $1.8(0.8)$ & $1.5(0.4)$ & $2.0(0.7)$ & $1.9(0.6)$ \\
\hline & Tension & $1.4(0.3)$ & $1.3(0.4)$ & $1.4(0.4)$ & $1.4(0.3)$ & $1.3(0.3)$ & $1.3(0.3)$ \\
\hline & Vigor & $3.3(0.6)$ & $2.7(0.7)$ & $3.1(0.7)$ & $3.6(0.6)$ & $2.9(0.7)$ & $2.8(0.8)$ \\
\hline
\end{tabular}

\section{Affective Go/No-Go}

Mean values and standard deviations are presented in Table 4.

Reaction Time (RT). Analysis of reaction time (RT) data revealed a main effect of Stress $[F(1,32)=5.73, p=.023]$, indicating longer RTs poststress compared to pre-stress, and a main effect of Shift $[F(1,32)=11.98$, $p=.002]$ with faster RTs for shift blocks than non-shift blocks. This Shift effect was qualified by a Treatment $X$ Shift interaction $[F(1,32)=5.5, p=$ .025], indicating faster RTs for shift trials than non-shift trials in the TRP+ condition only $[t(34)=4.31, p<.001]$ and a Valence $X$ Shift interaction $[F(1,32)=4.71, p=.038]$, indicating longer RTs for sad non-shift trials 
compared to sad shift trials $[t(34)=3.88, p<.001]$. Analysis also revealed a Valence X Genotype interaction $[F(1,32)=8.62, p=.006]$, reflecting significant longer RTs for sad words compared to happy words for the $S^{\prime} / S^{\prime}$ genotype group only $[t(17)=3.11, p=.006]$ (Figure 4).

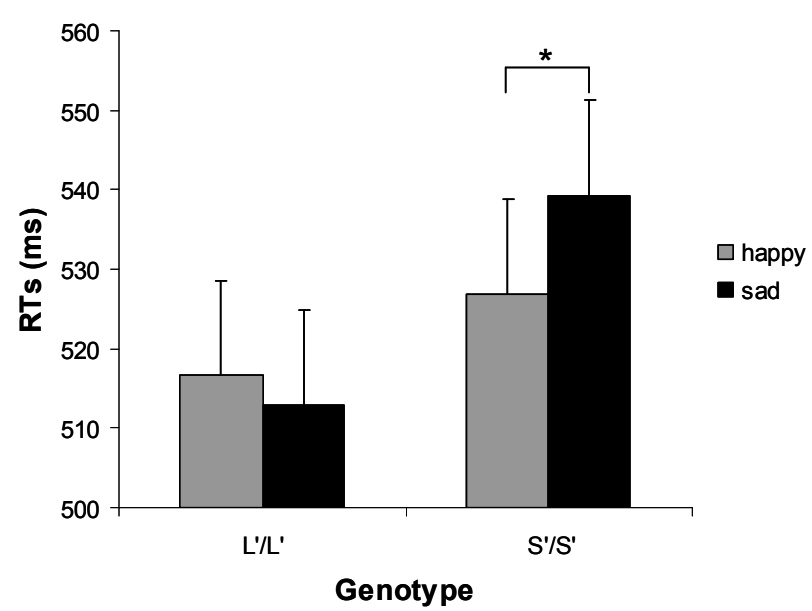

Figure 4. Mean RTs (SE) for happy and sad target words for both genotypes collapsed over stress showing significant longer RTs for sad words compared to happy words for the $S^{\prime} / S^{\prime}$ genotype group only $[t(17)=3.11, p=.006]$.

Errors. Analysis of error data revealed a main effect of Treatment $[F(1,32)=4.68, p=.038]$, indicating significantly more errors following TRP- than TRP+; a main effect of Stress $[F(1,32)=4.55, p=.041]$ with fewer errors post-stress than pre-stress and a main effect of Shift $[F(1,32)=21.99, p<.001]$ with more errors for shift than non-shift blocks. Furthermore, we found a Treatment X Genotype X Valence X Shift interaction $[F(1,32)=5.27, p=.028]$, which was explored by a 3-way (Treatment, Valence, Shift) ANOVA for each genotype revealing a Treatment $X$ Valence $X$ Shift interaction for the $S^{\prime} / S^{\prime}$ genotype only $[F(1,16)=12.24, p$ $=$.003]. To break down this interaction, a 2-way (Treatment, Valence) ANOVA for the $S^{\prime} / S^{\prime}$ genotype was run for each shift condition showing a Treatment $X$ Valence interaction for the shift condition only $[F(1,16)=9.19, p=.008]$, revealing more errors following TRP- than TRP+ during happy shift blocks [t(17) $=2.15, p=.047]$.

Further, a Treatment $X$ Stress $X$ Valence $X$ Shift interaction $[F(1,32)=13.01, p=.001]$ was found reflecting a Treatment $X$ Stress $X$ 
Valence interaction for the non-shift condition only $[F(1,33)=8.45, p=$ .006]. This interaction was further explored by a 2-way (Treatment, Stress) ANOVA for happy words and sad words yielding a Treatment $X$ Stress interaction for happy words only $[F(1,33)=12.62, p=.001]$, showing a decrease in errors pre-stress to post-stress following TRP$[t(34)=2.52, p=.017]$ but no effect following TRP $+[t(34)=1.67, p=.10]$.

Omissions. Analysis of omission data revealed a significant Treatment $X$ Valence $X$ Shift interaction $[F(1,32)=4.66, p=.038]$. A 2-way (Treatment, Valence) ANOVA was run for shift and non-shift blocks revealing a marginally significant Treatment $X$ Valence interaction for the shift blocks only $[F(1,33)=3.98, p=.054]$, indicating more omissions for happy shift trials following TRP+ compared to TRP-. However, this difference did not approach significance $[t(34)=1.78, p=.085]$. There were no effects of Genotype or Stress.

\section{Emotionally related free recall}

Mean values and standard deviations are presented in Table 4.

Immediate recall. Analysis of immediate recall data revealed a main effect of Valence $[F(2,64)=11.38, p<.001]$ and a significant Genotype X Treatment $X$ Valence interaction $[F(2,64)=4.17, p=.02]$. This interaction was explored by a two-way ANOVA (Treatment, Genotype) for positive, negative and neutral words, revealing a marginally significant Treatment $X$ Genotype interaction for positive words only $[F(1,32)=3.48, p=.071]$. This interaction, which is visualized in Figure 5, reflected that individuals with the $S^{\prime} / S^{\prime}$ genotype recalled fewer positive words following TRPthan $\mathrm{TRP}+[t(17)=2.5, p=.023]$. 


\section{Positive words}

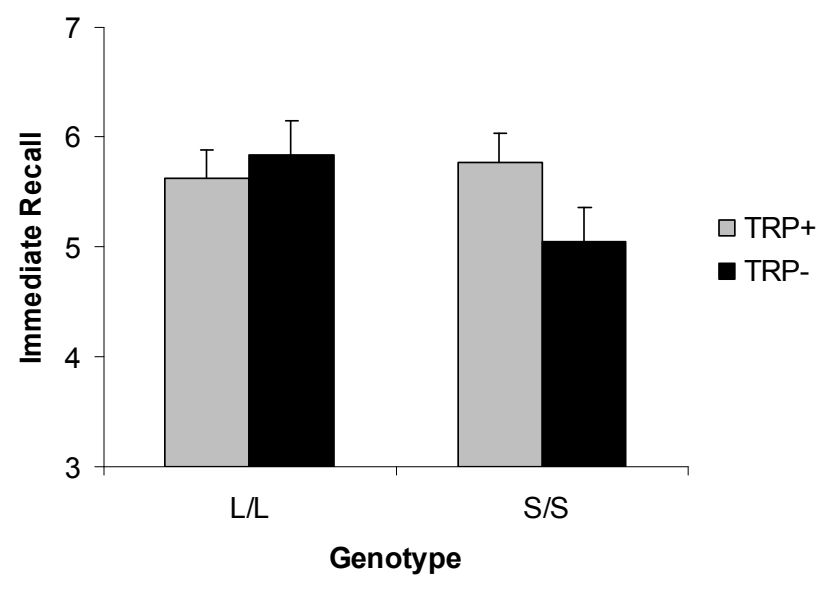

Figure 5. Mean number (SE) of immediately recalled positive words for both genotypes following TRP+ and TRP-collapsed over stress showing that individuals with the $\mathrm{S}^{\prime} / \mathrm{S}^{\prime}$ genotype recalled fewer positive words following ATD than $\operatorname{PLC}[t(17)=2.5, p=.023]$.

Delayed recall. An analysis of the delayed recall data revealed a main effect of Stress $[F(1,32)=14.57, p=.001]$ reflecting that fewer words were recalled post-stress than pre-stress, and a main effect of Valence $[F(2,64)=54.17, p<.001]$ showing that more positive words were recalled than negative and neutral words. Further, we found a significant Treatment $X$ Stress $X$ Valence interaction $[F(2,64)=3.28, p=.049]$. This interaction was further explored by a 2-way (Treatment, Stress) ANOVA for positive, negative and neutral words, revealing a Treatment $X$ Stress interaction for neutral words only $[F(1,33)=7.45, p=.01]$, indicating that more neutral words were recalled pre-stress compared post-stress following TRP $+[t(34)=4.37, p=.000]$ but not TRP- $[t(34)<1, n s]$. However, it should be noted that there was already a difference between treatments pre-stress $[t(34)=2.2, p=.034]$ with fewer neutral words being recalled following TRP- than TRP+. 


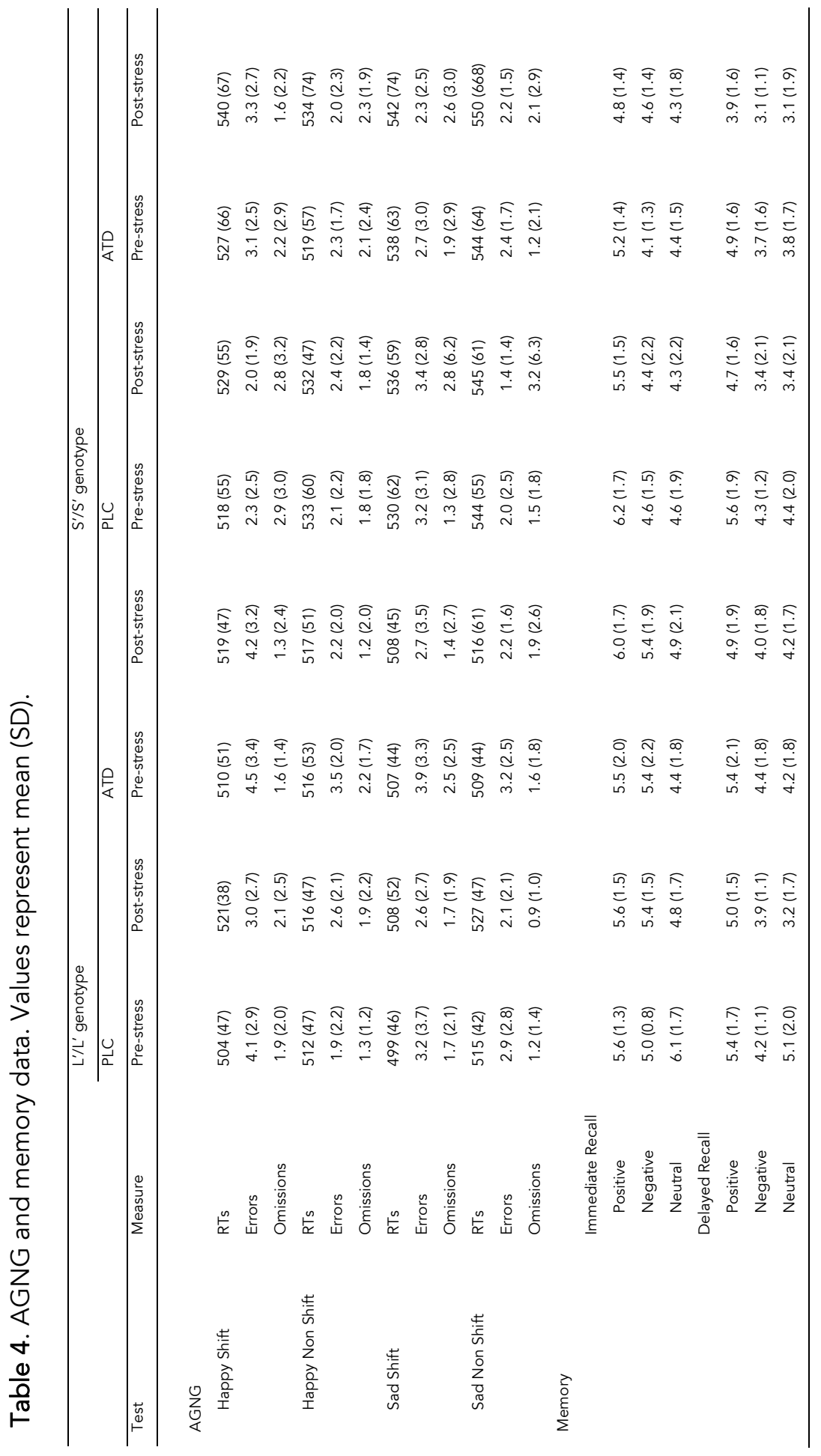




\section{DISCUSSION}

The goal of the present study was to assess mood, memory and attention in individuals with different 5-HTTLPR genotypes following ATD before and after the exposure to uncontrollable stress.

ATD lowered the plasma TRP:LNAA by $77 \%$, which is comparable with previous studies using the same collagen-protein (CP) ATD method (Evers et al., 2005) or the classic ATD mixture (Van der Does, 2001). Previous studies using the classic ATD mixture have reported an increase in plasma TRP of up to $200 \%$ in the balanced (TRP+) condition (for a review see Fusar-Poli et al., 2006), whereas the CP ATD method has been found to be more effective in maintaining a stable TRP+ condition (Evers et al., 2005; Firk \& Markus, 2008). In the present study, the TRP:LNAA ratio also increased by $62 \%$ in the TRP+ condition suggesting that the TRP+ condition may also be psychoactive due to an increase in $5-\mathrm{HT}$ synthesis. However, this increase in TRP:LNAA ratio is probably insufficient to affect behavior (Markus, 2008).

ATD resulted in a significant reduction of positive mood in all subjects independent of 5-HTTLPR genotype. This finding was surprising since most studies only found mood-lowering effects of ATD in individuals at risk for depression such as depressive patients in remission, individuals with a positive family history of depression $(\mathrm{FH}+$ ) (for a recent review see Ruhe et al., 2007) or in healthy s allele carries as compared to I carriers (Neumeister et al., 2002; Neumeister et al., 2006). However, few recent studies including the triallelic 5-HTTLPR polymorphism also revealed negative mood effects of ATD in I/I carriers if they were patients (Neumeister et al., 2006) or women (Walderhaug et al., 2007). Although our participants reported no family history of depression during screening, it may indeed be possible that some of these answers were instead based on lack of information, which may also explain this surprising effect. However, even if one or two young adult participants were actually $\mathrm{FH}+$, it is unlikely that this has confounded our findings given the sample size of the current study.

Even though the s allele is suggested as a vulnerability factor for the negative effects of ATD on mood, it seems that this is more complex and brain 5-HT manipulation may particularly alter mood in s allele carriers when the serotonergic system is actually needed in the face of acute 
stress as 5-HT plays a role in regulating HPA axis activity, which is important for stress coping (e.g. Porter et al., 2004). Therefore, we predicted that $\mathrm{s}$ allele carriers are especially vulnerable to ATD under acute stress exposure. For this purpose, the MPA task was used in the present study as an uncontrollable stress situation since it has frequently been found to induce stress as indicated by mood deterioration and increases in cortisol and electrophysiological arousal (e.g. Peters et al., 1998; Markus et al., 2002). Analysis indeed revealed a slight stress-induced increase in depression scores exclusively in $S^{\prime} / S^{\prime}$ genotypes, whereas in $L^{\prime} / L^{\prime}$ genotypes stress even increased positive feelings of vigor. These findings seem to support previous assumptions of 5-HTTLPR s allele as a vulnerability factor in the development of depression and suggest that $L^{\prime} / L^{\prime}$ carriers are less susceptible to the negative effects of stress than $S^{\prime} / S^{\prime}$ genotypes. As already described in the introduction, 5-HT plays an important role in stress coping which may explain why I allele carriers are more resistant to the negative effects of stress compared to $s$ allele carriers. This involvement of $5-\mathrm{HT}$ in stress coping is supported by complex interactions between 5-HT and the neuroendocrine stress system (Porter et al., 2004). Acute stress increases 5-HT neurotransmission (De Kloet et al., 1982; De Kloet et al., 1983; Davis et al., 1995), which promotes stress adaptation by mediating negative feedback control of cortisol on the HPA axis (Nuller \& Ostroumova, 1980; Van Praag, 2004). In accord with the current findings, s allele carriers are thought to be 5 -HT vulnerable and to be susceptible to depression symptoms particularly in the face of stress (Caspi et al., 2003; Van Praag, 2004; Firk \& Markus, 2007). Although hypothetically, stress and enhanced HPA activation, particularly in s allele carriers, may further undermine $5-\mathrm{HT}$ function, which then may lead to reduced stress coping and subsequently result in negative mood (e.g. Van Praag, 2004; Firk \& Markus, 2007; Dayan \& Huys, 2008). In further support, Gotlib (2008) found that only girls homozygous for the $s$ allele showed a cortisol response following acute stress exposure, which also supports the hypothesis that 5-HTTLPR affects HPA activity and hence, stress reactivity.

Although the affective effects of acute stress exposure appear to be influenced by 5-HTTLPR, there was no additional effect of ATD. Assuming that $S^{\prime} / S^{\prime}$ genotypes have a greater serotonergic vulnerability than $L^{\prime} / L^{\prime}$ genotypes, and that sufficient 5-HT function is a biological basis for stress coping, we expected that ATD would further increase the negative 
effect of stress exposure in $\mathrm{S}^{\prime} / \mathrm{S}^{\prime}$ genotypes. This absence of interaction between ATD, stress and 5-HTTLPR on depression scores might well be explained by the apparent modest effect of the current acute stress task. Although the MPA stress task also affected mood in the current study, cortisol responses were not increased following stress exposure, suggesting that the MPA task, in the present study, was only a brief and mild stressor. On the contrary, Gotlib et al (2008) found a 5-HTTLPR mediated change in cortisol responses by including a more severe and sustained stress procedure. Therefore, ATD may only mediate stressinduced affective changes in s allele carriers under highly emotionally (depression) relevant stressful conditions that require enhanced activation of 5-HT-involved adaptation mechanisms. Although in the current study design, stress may be confounded with fatigue effects over time, subjective mood ratings showed that fatigue decreases post-stress compared to pre-stress, suggesting that stress was not meaningfully confounded by fatigue over time in the current study.

Based on previous studies reporting memory (e.g. Rinck \& Becker, 2005) and attention bias (e.g. Murphy et al., 1999) toward negatively toned material in depressive patients, we expected a negative attention and memory bias for $S^{\prime} / S^{\prime}$ genotypes especially following ATD and stress exposure. On the AGNG task, we expected faster RTs when responding to sad words; however, slower RTs were found for sad words compared to happy words for $S^{\prime} / S^{\prime}$ genotypes. One possible explanation for this is that $S^{\prime} / S^{\prime}$ genotypes might have a pre-clinical baseline disposition to avoid negative information to counteract heightened amygdala responsivity to negative information (Hariri et al., 2005; Heinz et al., 2005; Pezawas et al., 2005). In support of this theory, avoidance behavior is generally recognized as a mediating factor in anxiety disorders and in the onset of depression (Tull \& Gratz, 2008). Another possible explanation might be that $\mathrm{S}^{\prime} / \mathrm{S}^{\prime}$ genotypes spend more time on the elaboration of negative material which might increase their vulnerability to depression. In support of this, healthy individuals without any vulnerability to depression actively avoid elaboration of negative stimuli while depressed patients fail to do so (Deveney \& Deldin, 2004). This is also consistent with a recently described neuro-computational model by Dayan and Huys (2008). In their model, 5-HT modulates behavioral inhibition as a Pavlovian control process suppressing negative representations that may lead to aversive states and consequences. By reducing brain 5-HT 
through ATD, this control process will fail and subsequently will give free rein to negative thoughts and actions. Further, in terms of this model, ATD might decrease suppression of negative thoughts particularly in $\mathrm{S}^{\prime} / \mathrm{S}^{\prime}$ genotypes due to serotonergic vulnerability that is caused by higher 5-HT levels throughout life span development as a function of less sufficient 5-HT reuptake (Dayan \& Huys, 2008). However, in the present study, ATD did not affect RTs, and increased the number of errors during happy shift blocks only in $\mathrm{S}^{\prime} / \mathrm{S}^{\prime}$ genotypes, suggesting a slightly increased ATD vulnerability for $S^{\prime} / S^{\prime}$ genotypes in line with our expectations. Previous findings from ATD studies in healthy subjects were inconsistent; some showed a negative attention bias following ATD (Murphy et al., 2002), others reported a bias toward positive material following ATD (Hayward et al., 2005) and others did not find a differential effect of ATD on emotional processing (Rubinsztein et al., 2001; Roiser et al., 2007).

On the memory task, ATD impaired immediate recall of positive words in $S^{\prime} / S^{\prime}$ genotypes. This indeed resembles the mood-congruent memory bias that has been reported for depressed patients reflecting impaired memory for positive information (Matt et al., 1992). Memory performance following ATD has been investigated in previous studies reporting impaired recall of positive words following ATD in healthy subjects (Klaassen et al., 2002) and impaired recall in healthy $S^{\prime} / S^{\prime}$ genotypes independent of word valence (Roiser et al., 2007). Thus, ATD impairs memory performance and in the present study even induces a depressive-like memory pattern in $S^{\prime} / S^{\prime}$ carriers following ATD, supporting greater vulnerability of $\mathrm{S}^{\prime} / \mathrm{S}^{\prime}$ carriers to depression.

To conclude, polymorphisms of the 5-HTTLPR differentially affects responses to mild stress and ATD, suggesting greater vulnerability of $\mathrm{S}^{\prime} / \mathrm{S}^{\prime}$ carriers to serotonergic manipulations and supporting increased depression vulnerability. The absence of interaction between ATD, stress and 5-HTTLPR, possibly due to the modest effect of the current acute stress task, merits further research using a stronger and a more sustained stress procedure. 


\section{CHAPTER 5}

Effect of different tryptophan sources on amino acids availability to the brain and mood in healthy volunteers

Based on:

Markus, C.R., Firk, C., Gerhardt, C., Kloek, J., Smolders, G.F. (2008). Effect of different tryptophan sources on amino acids availability to the brain and mood in healthy volunteers. Psychopharmacology, 201, 107-114. 


\section{ABSTRACT}

\section{Rationale}

Reduced brain serotonin (5-HT) function is acknowledged as a vulnerability factor for affective disturbances. Since the production of $5-\mathrm{HT}$ is limited by the availability of its plasma dietary amino acid precursor tryptophan (TRP), beneficial effects of TRP-rich alpha-lactalbumin whey protein (ALAC) have recently been studied. The effects of ALAC remain rather modest, and alternative protein sources of TRP may be more effective.

Objectives

We tested whether a TRP-rich hydrolyzed protein (HP) has greater effects on the plasma TRP:LNAA ratio and mood than intact ALAC protein in healthy volunteers.

\section{Methods}

In a double-blind, randomized cross-over study, plasma amino acids and mood were repeatedly measured in eighteen healthy subjects before and after intake of ALAC and HP as well as after placebo protein, pure TRP and a TRP-containing synthetic peptide. Except for the placebo protein, all interventions contained $0.8 \mathrm{~g}$ Trp.

Results

Significantly faster and greater increases in plasma TRP:LNAA were found after HP than after ALAC. In addition, the effects of HP on plasma TRP:LNAA were comparable with the effects of the TRP-containing synthetic peptide and even exceeded the effect of pure TRP. Sixty minutes after intake, mood was improved only following intake of HP and pure TRP, whereas longer lasting mood effects were only found after intake of HP.

Conclusion

The use of a TRP-rich hydrolyzed protein source may be more adequate to increase brain TRP and 5-HT function as compared with intact alphalactalbumin protein or pure TRP. 


\section{INTRODUCTION}

Brain serotonin (5-HT) receptors are widely distributed in the central nervous system and involve the regulation of several essential behavioral and physiological functions (e.g. mood, sleep, appetite, sexual behavior). Reduced 5-HT function is particularly recognized as a vulnerability factor involved in affective disorders, whereas increases in brain 5-HT are found to improve mood and to reduce affective disturbances in healthy and (sub)clinical subjects (Maes \& Meltzer, 1995; Markus et al., 1999; Markus, 2003; Van Praag, 2004).

Since $5-\mathrm{HT}$ is synthesised from the essential amino acid tryptophan (TRP), dietary manipulation of plasma TRP has been developed as a method to influence brain 5-HT synthesis. A significant increase in brain TRP and 5-HT can be accomplished by the intake of pure TRP, by increasing carbohydrate intake or by consumption of TRP-rich alphalactalbumin protein. These manipulations increase plasma concentrations of TRP to the sum of the other Large Neutral Amino Acids (TRP:LNAA ratio) and give TRP the advantage in competition for access into the brain (Fernstrom \& Wurtman, 1971; Fernstrom et al., 1973; Curzon, 1985). These dietary effects on the plasma TRP:LNAA ratio have been demonstrated frequently (Lieberman et al., 1986; Rosenthal et al., 1989; Markus et al., 1998; Markus, 2007) and clear associations have been found between plasma TRP:LNAA and brain 5-HT in animal as well as human studies after TRP depletion (Biggio et al., 1974; Carpenter et al., 1998; Nishizawa et al., 1997; Williams et al., 1999) and TRP augmentation (Fernstrom and Wurtman, 1971; Markus et al., 2000; Orosco et al., 2004).

Although these dietary methods may seem to be useful to improve $5-\mathrm{HT}$ related affective functioning by increasing brain $5-\mathrm{HT}$, their effects seem to be rather modest. Hence, a $20-25 \%$ increase in plasma TRP:LNAA as found with carbohydrate-rich diets does not seem to be sufficient enough to initiate meaningful behavioral changes (Markus et al., 1998; Markus et al., 1999). In addition, only modest effects of carbohydrates on mood are almost exclusively found in affected or sub-clinical subjects, whereas these effects are rather inconsistent or missing in healthy subjects (Markus, 2003). 
Supplementing food with pure TRP is in many cases limited by regulatory restrictions. However, the use of alpha-lactalbumin (ALAC) was recently introduced as a dietary method to further increase brain 5-HT and improve cognitive-affective behavior. ALAC is a whey-derived protein that contains a higher TRP content than most food protein sources (Heine et al., 1996). Recent studies reveal 50-130\% increases in plasma TRP:LNAA after ALAC intake (Markus et al., 2000a; Orosco et al., 2004; Markus et al., 2005; Merens et al., 2005; Booij et al., 2006; Scrutton et al., 2007). This TRP:LNAA increase improves brain 5-HT function (Markus et al., 2000a; Orosco et al., 2004) and mood or stress-related affective behavior (Markus et al., 2000a). It even reduces sleep disturbances in rats (Minet-Ringuet et al., 2004) and causes sleep-related improvement in behavioral and brain measures of concentration in human subjects (Markus et al., 2005). Using ALAC has a clear nutritional advantage over pure TRP and carbohydrates and can also be given for a longer period of time through daily consumption. Nevertheless, the beneficial effects of ALAC on cognitive-affective behaviour remain to be rather modest and are not always clearly found (Merens et al., 2005). It may be that the increase in plasma TRP:LNAA by ALAC is too small to cause meaningful behavioral changes. Instead of using intact ALAC protein, a hydrolyzed protein with a TRP:LNAA ratio superior to ALAC or other food sources of TRP may lead to faster and greater increases in plasma TRP:LNAA and may therefore also cause faster and stronger increases in brain 5-HT and affective changes.

The aim of the present study was to investigate the effects on plasma TRP:LNAA ratio and mood after consumption of a TRP-rich hydrolyzed protein (HP) as compared to other sources of TRP by healthy volunteers. During five experimental sessions, plasma amino acid concentrations were measured before and several times after intake of intact ALAC, HP and placebo protein, as well as after pure TRP and a TRP-containing synthetic peptide as positive controls. In addition, mood was measured before and after intake. All of the interventions (except the placebo) contained similar amounts of TRP, but differed in their content of other amino acids. 


\section{METHODS}

\section{Subjects}

Eighteen healthy students ( 9 males and 9 females: age between 1830 years) at Maastricht University participated in the study. Exclusion criteria for participation were chronic and current illness; history of psychiatric or medical illness; medication use; metabolic, hormonal or intestinal diseases; irregular diets or deviant eating habits; excessive use of alcohol (>2 units a day), cigarettes, coffee and/or drugs; aversion for sugar-rich products; pregnancy; and any discomfort with blood drawing procedures; as assessed by health and lifestyle questionnaires. Subjects participating in the experiment had a body-mass index in the normal range (BMI in $\mathrm{kg} / \mathrm{m}^{2}$ between 20-25) and female subjects were matched for contraception. Women participated during their mid-late follicular phase (day 4-10), while women using contraception participated when they actually used the contraception pill. All subjects that participated in the experiment were non-smokers and non-drug users and were not allowed to drink alcohol starting two days before the experiment until the end of the experiment. The study was approved by the Medical Ethics Committee of the Academic Hospital Maastricht (Maastricht, The Netherlands) and the procedures followed were in accordance with the Helsinki Declaration of 1975 as revised in 1983. All subjects gave their signed informed consent to participate in the experiment prior to their inclusion in the experiment and received a financial compensation for their participation.

\section{Procedure}

Approximately 600 students at Maastricht University received written information about the study and an invitation to participate. In addition, students who were interested to participate were requested to complete and return a questionnaire package concerning general information (health, smoking and drinking habits, caffeine consumption, weight and height, and use of psychoactive drugs) and several questionnaires concerning psychopathology (mood disorders, family history of depression, medical complaints, etc). From the subjects showing interest in taking part in the experiment, eighteen healthy subjects were selected for the 
experiment. Subsequently, subjects taking part in the experiment were invited at the lab to receive information about the study and to become familiar with the environment and experimental procedures.

During five experimental morning sessions, subjects visited the laboratory to monitor plasma TRP:LNAA concentrations and mood following intake of a drink containing different TRP:LNAA ratios (Figure 1A). The order of presentation of dietary conditions was counterbalanced and the four experimental days were separated by a one-week period. Before and 15, 30, 60, 90 120, 180 and 210 minutes after ingestion, blood samples were taken to measure the dose dependent effect (response-curve) of the different TRP or protein sources on plasma amino acid concentrations and the TRP:LNAA ratio. In addition, mood was measured before, 60 minutes and 210 minutes after intake (Figure 1B).

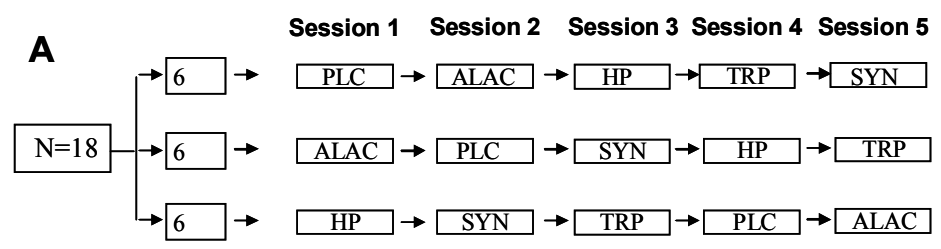

B

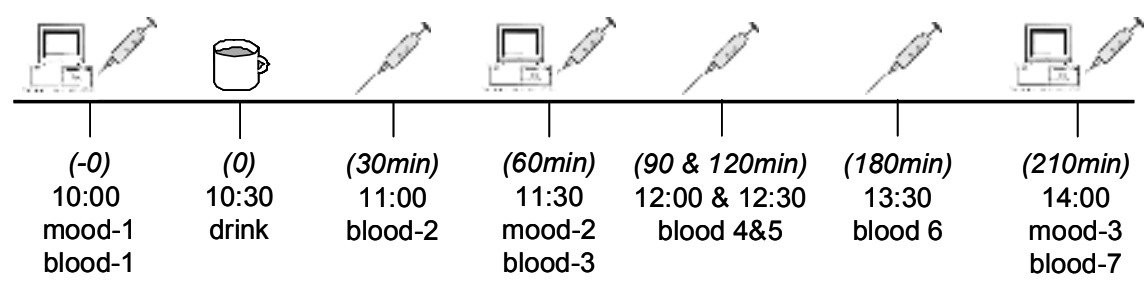

Figure 1. General design (A) and time schedule (B) of the experiment.

On each experimental morning, six subjects arrived at the laboratory at $0830 \mathrm{am}$. Subjects had been instructed to fast overnight; only water or tea without sugar was permitted. In addition, subjects were not allowed to use any kind of drugs before and during the experiment (see selection criteria) or to drink alcohol the day before their participation and arrival at the laboratory. After arrival, subjects were allowed to rest for a while before an indwelling catheter was inserted in their non-preferred forearm. Then all subjects were brought into a laboratory room containing 
sitting areas with separated computer systems. Each subject was separately seated in front of his or her personal computer screen and instructed about the experiment. Subsequently, subjects were exposed to a computerized version of the Profile of Mood state (POMS) (see for detailed description below). After the first test session, subjects received a test drink containing different TRP:LNAA ratios. Before, 60 minutes and 210 minutes after intake respectively a first, second and third mood measure was conducted. Before and 15, 30, 60, 90 120, 180 and 210 minutes after intake, blood samples were taken to measure the dietary effects on plasma amino acid concentrations. Between measurements, all subjects stayed in the laboratory room to read or work on their own private computer.

\section{Diets}

On each experimental morning, a $312 \mathrm{ml}$ drink was consumed containing different TRP or LNAA concentrations. The reference condition included 20gram casein protein (PLC: DSM Delft; The Netherlands) with $0.4 \mathrm{~g}$ TRP and $10 \mathrm{~g}$ LNAA, whereas the test conditions included $15 \mathrm{gram}$ intact alpha-lactalbumin (ALAC) whey protein (BioPURE, Davisco Foods International, Inc.; USA) with $0.8 \mathrm{~g}$ TRP and $9.4 \mathrm{~g}$ LNAA, TRP-rich hydrolyzed protein (HP) (PeptoBalance ${ }^{\mathrm{TM}}$, DSM Delft; The Netherlands) with $0.8 \mathrm{~g}$ TRP and $4 \mathrm{~g}$ LNAA, $0.8 \mathrm{~g}$ pure TRP (TRP: Orthica, Almere; The Netherlands) and $1.2 \mathrm{~g}$ synthetic peptide (SYN: Ser-Trp, DSM Delft; Molecular formula $\mathrm{C}_{14} \mathrm{H}_{17} \mathrm{~N}_{3} \mathrm{O}_{4}$ ) containing $0.8 \mathrm{~g}$ tryptophan (Tables 1 and 2).

Table 1. Composition of the standard protein (PLC), alpha-lactalbumin whey protein (ALAC), TRP-rich hydrolyzed protein (HP), pure TRP and synthetically depeptide (SYN) condition.

\begin{tabular}{llll}
\hline Source & Product & TRP & $\begin{array}{l}\text { TRP:LNAA } \\
\text { (mol/mol) }\end{array}$ \\
\hline PLC & $20 \mathrm{~g} / 312 \mathrm{ml}$ & 0.4 & 0.04 \\
ALAC & $15 \mathrm{~g} / 312 \mathrm{ml}$ & 0.8 & 0.1 \\
HP & $312 \mathrm{ml}$ & 0.8 & 1.1 \\
pure TRP & $0.8 \mathrm{~g} / 312 \mathrm{ml}$ & 0.8 & $\infty$ \\
SYN & $1.2 \mathrm{~g} / 312 \mathrm{ml}$ & 0.8 & $\infty$ \\
\hline
\end{tabular}


Table 2. Amino acid profile of the standard protein (PLC), alpha-lactalbumin whey protein (ALAC) and protein hydrolysate (HP) condition.

\begin{tabular}{llll}
\hline Amino acid profile $(\mathrm{g})$ & $\mathrm{HP}$ & $\mathrm{PLC}$ & ALAC \\
\hline Isoleucine & 1.004 & 1.800 & 2.012 \\
Leucine & 0.745 & 2.733 & 3.306 \\
Phenylalanine & 0.480 & 1.467 & 1.309 \\
Tyrosine & 1.216 & 1.867 & 1.451 \\
Valine & 0.554 & 2.133 & 1.322 \\
Total LNAA & 3.999 & 10 & 9.4 \\
Tryptophan & 0.8 & 0.4 & 0.8 \\
\hline
\end{tabular}

All drinks are prepared by mixing the powder with $0.10 \mathrm{~g}$ sweetener (acesulfame) and were filled up by plaine water in order to reach a $312 \mathrm{~mL}$ drink. Research assistants blind to the dietary conditions conducted the administration of the different drinks.

\section{Measurements}

Profile of Mood States (POMS). Changes in mood are measured using a computerized Dutch shortened version of the POMS questionnaire (Wald \& Mellenbergh, 1990) as a VAS scale ranging from 'strongly disagree' to 'strongly agree'. The POMS was calculated by the total mood scores on five different subscales; ranging from Anger, Depression, Fatigue and Tension that refer to a negative mood state, to Vigor concerning a positive mood.

\section{Biochemical Analyses}

Blood samples were collected in duplicate in $5 \mathrm{ml}$ Vacutainer tubes containing sodium heparin and centrifuged at $5000 \mathrm{rpm}$ for $5 \mathrm{~min}$ at $4^{\circ} \mathrm{C}$. Subsequently, the supernatants were directly stored at $-80^{\circ} \mathrm{C}$ until analysis. Before storage, the supernatant $(100 \mu \mathrm{l})$ was mixed with $4 \mathrm{mg}$ sulfasalicyl acid. Plasma amino acid analysis was conducted with High pressure liquid chromatography (HPLC), making use of a 2-3 $\mu \mathrm{m}$ Bischof Spherisorb ODS II column. The plasma TRP:LNAA ratio was calculated by dividing the plasma TRP concentration (in $\mu \mathrm{mol} / L$ ) by the sum of the other LNAA's, i.e. valine, isoleucine, leucine, tyrosine and phenylalanine. 


\section{Experimental design and statistical analysis}

The main research questions formulated in the introduction were analyzed by means of repeated measures multivariate and univariate analyses of variance (MANOVA and ANOVA) by using the General Linear Model (GLM: SPSS 12.0 for Windows) with one within-subjects factors "Condition" (5 different conditions: PLC, ALAC, HP, pure TRP, SYN) on the several dependent measures from mood and plasma amino acids. Although we already counterbalanced for order of condition, this variable was preliminarily taken as a between-subjects factor. Yet, because order of intervention did not contribute to any of the results, final analyses were performed with only Condition as a within-subjects factor. For the effect of diet manipulation on blood samples, repeated measures multivariate analyses of variance was performed; with first- and second order polynomial contrasts. Only significant results revealed by these procedures were further examined by univariate tests. Huynh-Feldt or Greenhouse-Geisser corrected P values, their corresponding epsilons as well as the original, i.e. uncorrected, degrees of freedom are reported when the sphericity assumption was not met. The study, including validation of the group size, was designed to detect a large effect size $\left(\mu^{2} 0.20\right)$ for a power of 0.80 at alpha $=0.05$ (requiring a group size of at least seventeen subjects). All statistics were evaluated at a significance level of $5 \%$. Data are reported as means \pm SD

\section{RESULTS}

\section{Plasma TRP:LNAA}

A first repeated measures analysis of variance with Condition (PLC, ALAC, HP, pure TRP, SYN) and Time $(0,30,60,90,120,180$ and 210 $\mathrm{min}$ ) as within-subjects factors on the plasma TRP:LNAA ratio revealed a main significant effect of Time $[F(6,90)=122 ; P<0.0001]$ and Condition $[F(4,60)=79,68 ; P<0.0001]$ and a significant interaction between Condition and Time $[F(24,360)=41,50 ; P<0.0001]$; indicating that the increase in plasma TRP:LNAA ratio across time differed between conditions. As shown in Figure 2, the highest significant increases in plasma TRP:LNAA 
ratio were found $30-120 \mathrm{~min}$ after $\operatorname{SYN}[F(6,96)=87,89 ; \mathrm{P}<0.0001]$ and $\mathrm{HP}$ $[F(6,90)=86,54 ; P<0.0001]$.

\section{TRP/LNAA}

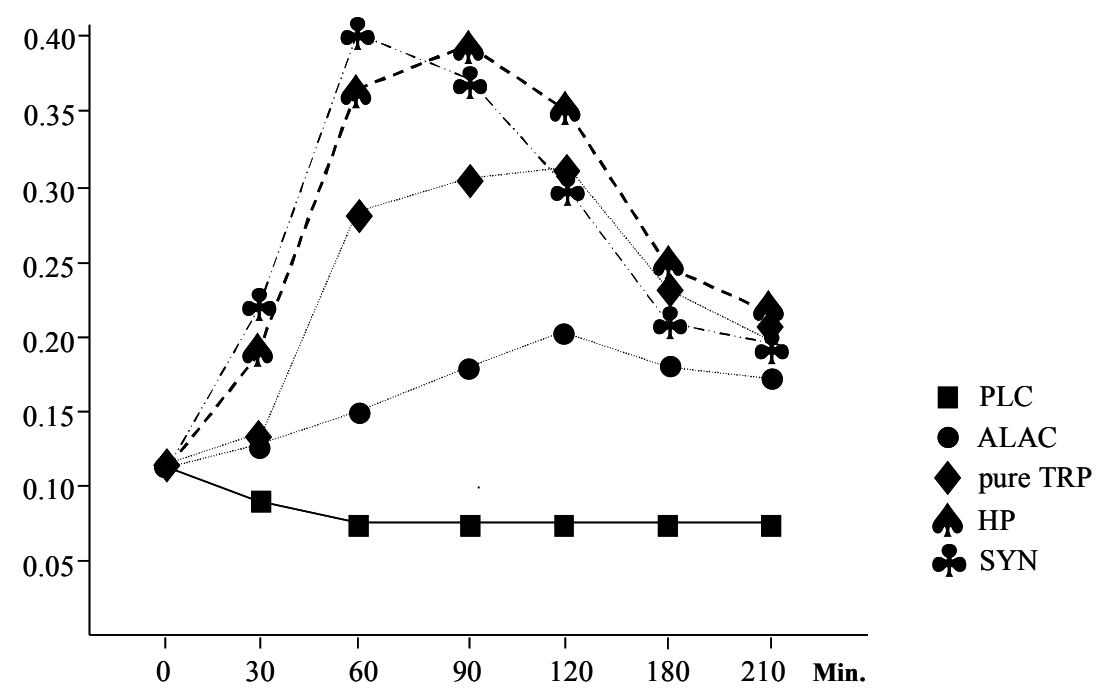

Figure 2. Mean changes in plasma tryptophan as compared with the other large neutral amino acids ratio (TRP:LNAA) after intake of hydrolysed protein (HPROT), alphalactalbumin (ALAC), casein protein (PLC), pure tryptophan (TRP) and tryptophan-containing synthetic peptide (SYN). A multivariate significant interaction of Condition by Time $[P<0.0001]$ revealed the highest and fastest significant increases in plasma TRP:LNAA ratio 30-120min after SYN $[P<0.0001]$ and HPROT $[P<0.0001]$.

There were no significant differences in peak TRP:LNAA responses between these two conditions; with the exception that peak (263\%) increases in TRP:LNAA after SYN appeared significantly faster (after 60 min) than peak (255\%) increases after HP (after $90 \mathrm{~min}$ ) $[F(3,14)=3,66$; $P=0.04]$. The increase in TRP:LNAA after HP and SYN was significantly faster and higher than after pure $\operatorname{TRP}[F(4,60)=4,56 ; P=0.003]$. With pure TRP, a peak $191 \%$ increase in TRP:LNAA was found 120 min after intake $(P<0.0001)$. A significant increase in plasma TRP:LNAA was also found $60-210$ min after $\operatorname{ALAC}[F(6,96)=72 ; P<0.0001]$, with a peak $67 \%$ increase $120 \mathrm{~min}$ after intake $(\mathrm{p}<0.0001)$, but this was significantly lower as compared with HP $[F(4,60)=60,4 ; P<0.0001]$ and pure TRP $[F(4,64)=30,83$; $P<0.0001]$. After PLC, there was a significant decline in TRP:LNAA starting $60 \mathrm{~min}(-27 \%)$ until $210 \mathrm{~min}(<0.001)$ after intake $[F(6,96)=32,95$; 
$\mathrm{P}<0.0001]$. The amino acid concentrations of the different protein and peptide sources across time are given in Table 3 and the plasma TRP:LNAA ratios of different conditions across time are given in Table 4.

Table 3. Plasma amino acid concentrations $(\mu \mathrm{m} / \mathrm{L}$ ) across time after protein (PLC), alphalactalbumin protein (ALAC), protein hydrolysate (HPROT), pure tryptophan (TRP) and synthetically depeptide (SYN)

\begin{tabular}{|c|c|c|c|c|c|c|c|c|}
\hline \multirow[b]{2}{*}{ Amino Acid } & \multirow[b]{2}{*}{ Condition } & \multicolumn{7}{|l|}{ Time } \\
\hline & & to & $\mathrm{t} 30$ & t60 & t90 & t120 & t180 & $\mathrm{t} 210$ \\
\hline \multirow[t]{5}{*}{ Isoleucine } & PLC & 0.07 & 0.10 & 0.18 & 0.15 & 0.12 & 0.09 & 0.08 \\
\hline & ALAC & 0.08 & 0.12 & 0.20 & 0.22 & 0.18 & 0.12 & 0.11 \\
\hline & $\mathrm{HP}$ & 0.07 & 0.09 & 0.09 & 0.14 & 0.09 & 0.08 & 0.09 \\
\hline & pure TRP & 0.07 & 0.07 & 0.07 & 0.06 & 0.07 & 0.07 & 0.07 \\
\hline & SYN & 0.06 & 0.07 & 0.06 & 0.06 & 0.06 & 0.06 & 0.07 \\
\hline \multirow[t]{5}{*}{ Leucine } & PLC & 0.12 & 0.19 & 0.31 & 0.26 & 0.22 & 0.17 & 0.16 \\
\hline & ALAC & 0.13 & 0.22 & 0.37 & 0.38 & 0.28 & 0.21 & 0.20 \\
\hline & $\mathrm{HP}$ & 0.13 & 0.14 & 0.14 & 0.13 & 0.13 & 0.13 & 0.14 \\
\hline & pure TRP & 0.13 & 0.13 & 0.12 & 0.12 & 0.12 & 0.12 & 0.13 \\
\hline & SYN & 0.13 & 0.14 & 0.12 & 0.12 & 0.12 & 0.12 & 0.13 \\
\hline \multirow[t]{5}{*}{ Phenylalanine } & PLC & 0.06 & 0.08 & 0.10 & 0.08 & 0.08 & 0.06 & 0.06 \\
\hline & ALAC & 0.07 & 0.09 & 0.11 & 0.10 & 0.09 & 0.07 & 0.07 \\
\hline & $\mathrm{HP}$ & 0.07 & 0.07 & 0.07 & 0.06 & 0.10 & 0.06 & 0.07 \\
\hline & pure TRP & 0.07 & 0.07 & 0.06 & 0.06 & 0.06 & 0.06 & 0.06 \\
\hline & SYN & 0.06 & 0.07 & 0.06 & 0.06 & 0.06 & 0.06 & 0.06 \\
\hline \multirow[t]{5}{*}{ Tyrosine } & PLC & 0.06 & 0.07 & 0.12 & 0.11 & 0.09 & 0.07 & 0.07 \\
\hline & ALAC & 0.06 & 0.08 & 0.12 & 0.12 & 0.10 & 0.08 & 0.08 \\
\hline & $\mathrm{HP}$ & 0.06 & 0.07 & 0.07 & 0.06 & 0.06 & 0.06 & 0.06 \\
\hline & pure TRP & 0.06 & 0.06 & 0.06 & 0.05 & 0.06 & 0.05 & 0.05 \\
\hline & SYN & 0.05 & 0.06 & 0.05 & 0.05 & 0.05 & 0.05 & 0.05 \\
\hline \multirow[t]{5}{*}{ Valine } & PLC & 0.24 & 0.28 & 0.45 & 0.42 & 0.38 & 0.32 & 0.30 \\
\hline & ALAC & 0.26 & 0.30 & 0.38 & 0.42 & 0.35 & 0.29 & 0.28 \\
\hline & $\mathrm{HP}$ & 0.26 & 0.27 & 0.25 & 0.25 & 0.25 & 0.25 & 0.26 \\
\hline & pure TRP & 0.25 & 0.25 & 0.23 & 0.22 & 0.23 & 0.22 & 0.23 \\
\hline & SYN & 0.21 & 0.26 & 0.22 & 0.22 & 0.22 & 0.21 & 0.23 \\
\hline \multirow[t]{5}{*}{ Tryptophan } & PLC & 0.06 & 0.07 & 0.08 & 0.08 & 0.07 & 0.06 & 0.05 \\
\hline & ALAC & 0.07 & 0.09 & 0.18 & 0.23 & 0.19 & 0.13 & 0.12 \\
\hline & $\mathrm{HP}$ & 0.07 & 0.13 & 0.21 & 0.23 & 0.20 & 0.14 & 0.13 \\
\hline & pure TRP & 0.07 & 0.07 & 0.17 & 0.18 & 0.18 & 0.13 & 0.11 \\
\hline & SYN & 0.06 & 0.13 & 0.21 & 0.18 & 0.15 & 0.11 & 0.10 \\
\hline
\end{tabular}


Table 4. Plasma TRP:LNAA ratios $(\mu \mathrm{m} / \mathrm{L}$ ) across time after protein (PLC), alpha-lactalbumin protein (ALAC), TRP-rich hydrolyzed protein (HP), pure TRP and synthetically depeptide (SYN).

\begin{tabular}{lllllllll}
\hline & & \multicolumn{7}{l}{ Time } \\
\cline { 3 - 8 } Plasma ratio & Condition & t0 & t30 & t60 & t90 & t120 & t180 & t210 \\
\hline TRP:LNAA & PLC & 0.11 & 0.09 & 0.08 & 0.08 & 0.08 & 0.08 & 0.08 \\
& ALAC & 0.12 & 0.12 & 0.15 & 0.18 & 0.2 & 0.18 & 0.17 \\
& HP & 0.11 & 0.19 & 0.36 & 0.39 & 0.35 & 0.25 & 0.22 \\
& pure TRP & 0.11 & 0.12 & 0.29 & 0.31 & 0.32 & 0.24 & 0.20 \\
& SYN & 0.11 & 0.22 & 0.40 & 0.37 & 0.31 & 0.22 & 0.19 \\
\hline
\end{tabular}

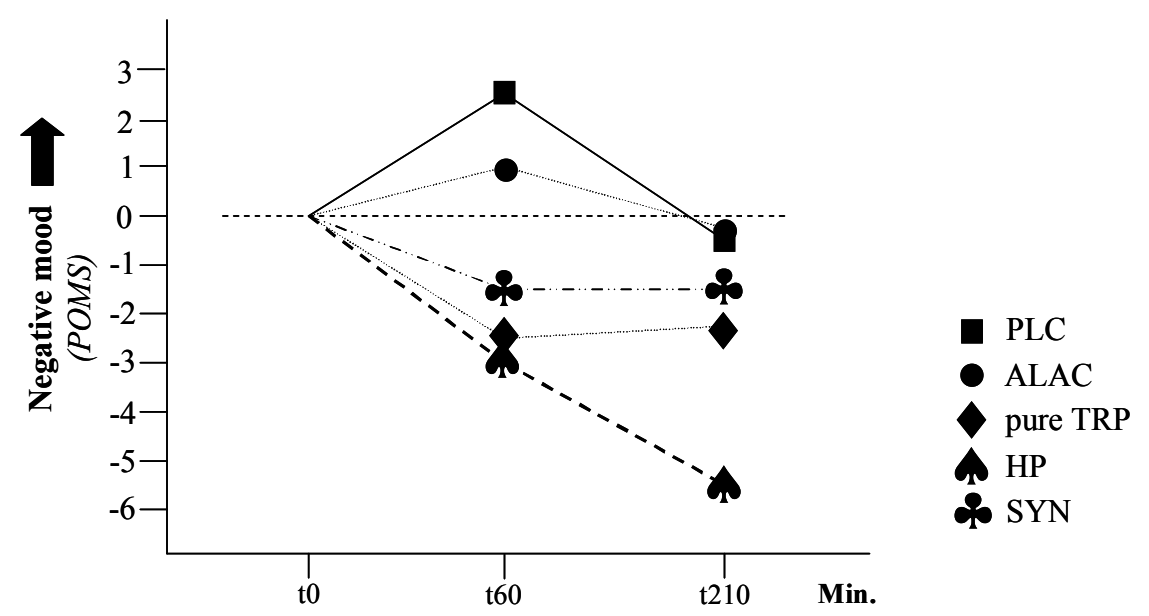

Figure 3. Mean differences in the total mood changes of the Profile Of Mood State (POMS) after intake of TRP-rich hydrolyzed protein (HP), alpha-lactalbumin (ALAC), casein protein (PLC), pure TRP and TRP-containing synthetic peptide (SYN). A significant interaction of Condition by Time $[P=0.009]$ indicated comparable mood improvements $60 \mathrm{~min}$ after HP $(P=015)$ and pure TRP $(P=0.010)$ with the most durable mood improvements following HP 210min after intake $(P=0.04)$.

\section{Mood}

Repeated measures analysis of variance with Condition (PLC, ALAC, HP, pure TRP, SYN) and Time $(0,60$ and $210 \mathrm{~min})$ as within-subjects factors on the total mood scores revealed a significant effect of Time $[F(2,32)=3,27 ; P<0.05]$ and a significant interaction of Condition $\times$ Time $[F(4,64)=3,68 ; P=0.009]$; indicating that mood changes across time sig- 
nificantly differed between conditions. As shown in Figure 2, comparable improvements of mood were found $60 \mathrm{~min}$ after HP $(P=0.015)$ and pure TRP $(P=0.010)$ but only after HP mood further improved 210min after intake as compared with pure TRP $(P=0.04)$. In contrast, no mood changes were found after PLC $(P>0.2)$ and ALAC $(P=0.2)$. Although mood also seem to improve after $S Y N$, this was not significant $(P>0.2)$.

\section{DISCUSSION}

The aim of the present study was to investigate whether a TRP-rich hydrolyzed protein (HP) has a greater effect on temporal increases in plasma TRP:LNAA and mood as compared with intact alpha-lactalbumin (ALAC) protein in healthy volunteers. In addition, a TRP-containing synthetic peptide and pure TRP were included as positive controls. Significantly faster and greater increases in plasma TRP:LNAA were found after HP intake than after intake of ALAC. In addition, the effects of HP on plasma TRP:LNAA were comparable with the effects of the TRPcontaining synthetic peptide and even exceeded the effect of pure TRP. Mood improved after HP and pure TRP 60 min after intake, and the most profound and durable mood improving effects were found with HP 210 min after intake.

In general, the changes of the TRP:LNAA ratios were consistent with the kinetics of TRP concentrations reported earlier (Domino, 1976; Yuwiler et al., 1981). There was a significantly greater increase in plasma TRP:LNAA after intake of HP (255\%) as compared with ALAC (67\%). This effect of HP on plasma TRP:LNAA was comparable with the TRPcontaining synthetic peptide (SYN) and even largely exceeded the 191\% increase in plasma TRP:LNAA induced by pure TRP. Since all sources (except the negative control) contained equal amounts of TRP, it was anticipated that the differences found in plasma TRP:LNAA ratios should be due primarily to differences in LNAA content between the sources. Whereas this notion is substantiated by the results from the natural TRP sources, the findings from pure TRP and SYN show that other factors may play a role as well. Both, pure TRP and SYN have an infinitely high TRP:LNAA ratio, but SYN results in a substantially higher plasma TRP:LNAA ratio than pure TRP. We speculate that TRP in the form of small peptides has a better bioavailability than pure TRP as such. It has been suggested that a $40-70 \%$ variation in plasma TRP:LNAA could be 
sufficient to change brain $5-\mathrm{HT}$ content in animal as well as human; either reducing brain 5-HT by lowering plasma TRP:LNAA after TRP depletion (Biggio et al., 1974; Carpenter et al., 1998; Nishizawa et al., 1997; Williams et al., 1999) and increasing 5-HT by enhancing plasma TRP:LNAA after TRP augmentation (Fernstrom and Wurtman, 1971; Markus et al., 2000; Orosco et al., 2004) or increasing carbohydrates in normal meals. Since the current $255 \%$ rise in TRP:LNAA induced by consumption of HP largely exceeds this threshold, and also based on findings that TRP suppletion affected mood and behaviour in human (Markus et al., 2000; see for a review Markus, 2008), it is very likely that this causes a much greater rise in available brain TRP and 5-HT and a subsequent stronger psychological-emotional effect in the brain.

A significant improvement in mood was found one hour after consumption of HP, and intake of pure TRP. Moreover, only HP was able to induce a sustained mood-improvement until 210 min after intake. No significant effects were found with ALAC or SYN. The absence of a mood effect after consumption of ALAC is comparable with previous studies, showing that ALAC only induces beneficial effects on mood in stressvulnerable subjects under acute stress exposure (Markus et al., 2000a; Markus, 2003). Current results suggest that a large $255 \%$ increase in plasma TRP:LNAA may be sufficient to improve baseline mood in nonvulnerable subjects as well. Nevertheless, the effects of HP may still be more profound in 5-HT-vulnerably subjects and/or when brain TRP supply is actually required. People differ in their sensitivity to alterations in the serotonergic system, and among the different factors involved in such brain 5-HT vulnerabilities, genetic proneness and chronically stressful circumstances are the most frequently described (Markus et al., 2000a; Markus, 2003; Firk \& Markus, 2007; Jans et al., 2007). Thus, most beneficial effects of brain TRP supply may be expected in '5-HTvulnerable' subjects like people with a family history of depression, carrying the susceptible short-allele serotonin transporter gene (5-HTTLPR), in chronically stressed subjects (Markus et al., 2000a; Markus, 2003; Firk \& Markus, 2007; Jans et al., 2007) or in clinically depressed or remitted subjects who respond to selective serotonin reuptake inhibitors (SSRI's). This intriguing hypothesis certainly deserves further investigation.

Contrary to our expectations, there were no significant improvements in mood after intake of SYN. Although mood tended to be improved after SYN as compared with PLC and ALAC, this effect was not 
statistically significant. This is surprising since the increase in plasma TRP:LNAA following intake of SYN was comparable to the increase found after consumption of HP and even exceeded the 191\% increase in plasma TRP:LNAA as found after pure TRP. Although hypothetically, it may be that the brain bioavailability of TRP released from a synthetic dipeptide is somehow reduced. Alternatively, the power of the current study may not have been sufficient to detect a significant difference in the SYN condition.

Current findings support the assumption that the use of a hydrolyzed TRP-rich protein may be a more adequate method to increase central TRP and 5-HT levels and brain function as compared to intact ALAC or pure TRP and, hence, to further improve 5-HT related affective behavior. Although the beneficial affective effects of TRP supply appear to be rather negligible in healthy subjects under normal circumstances, it is anticipated that the effects of the current HP may become more vivid when chronically administered, under high affecting circumstances that require enhanced TRP supply, and in clinically or 5-HT-vulnerable subjects who are responsive to brain $5-\mathrm{HT}$ manipulations. This should be explored in future research. 



\section{CHAPTER 6}

Mood and cortisol responses following tryptophan augmentation and acute stress in healthy subjects with high and low cognitive reactivity to depression

Based on:

Firk, C., Markus, C.R. (in revision). Mood and cortisol responses following tryptophan augmentation and acute stress in healthy subjects with high and low cognitive reactivity to depression. 


\section{ABSTRACT}

\section{Background}

It is suggested that stress particularly in subjects with high cognitive reactivity (CR), a psychological vulnerability marker of depression, may increase or even induce serotonergic vulnerability, which in turn may lead to reduced serotonin (5-HT) function, decreased stress-coping and an increased risk to develop depressive symptoms.

\section{Objective}

The aim of the present study was to investigate the beneficial effects of 5-HT augmentation through a tryptophan-rich hydrolyzed protein (HP) on mood and stress coping in subjects with high and low CR. We hypothesized that subjects with high CR are more responsive to the beneficial effects of HP than subjects with low CR particularly after acute stress exposure.

\section{Design}

In a placebo-controlled, double-blind, crossover study, participants' mood and cortisol was assessed before and after acute stress exposure either following intake of HP or placebo.

\section{Results}

HP significantly increased positive mood in all subjects and dampened the cortisol response to acute stress. No differences were found between high and low CR subjects.

\section{Conclusions}

To conclude, dietary 5-HT augmentation with HP has beneficial effects on mood and stress-coping also in healthy non-vulnerable subjects suggesting that HP may be a more adequate method to augment TRP and brain 5-HT compared to previously used TRP-rich proteins and to improve 5 -HT-related stress resilience. 


\section{INTRODUCTION}

Serotonin (5-Hydroxytryptamine, 5-HT) is involved in almost every conceivable physiological or behavioral function as for example in the regulation of emotion, sleep, appetite and aggression (Aghajanian \& Sanders-Bush, 2002). Therefore serotonergic dysfunction is implicated in many psychological disorders and it is widely accepted that $5-\mathrm{HT}$ plays a key role in the onset and course of depression (Maes \& Meltzer, 1995; Neumeister et al., 2004c). Evidence comes from studies reporting lower plasma 5-HT precursor availability of tryptophan (TRP) for the brain, reduced cerebrospinal fluid (CSF) concentration of the serotonin metabolite 5-hydroxyindoleacetic acid (5-HIAA) and decreased platelet 5-HT uptake in depressive patients, suggesting involvement of diminished brain 5-HT function in the onset of depression symptoms (Maes \& Meltzer, 1995; Neumeister et al., 2004c). However, in spite of the fact that serotonergic dysfunction plays a major role in depression, it is unlikely that this neurotransmitter is entirely responsible for its pathogenesis (Maes \& Meltzer, 1995)

In the search for additional factors involved in the development of depression, stress has received particular attention. Stressful life events often precede the onset of depression (Brown et al., 1987; Heim \& Nemeroff, 2001; Van Praag, 2004) and individuals with a genetic serotonergic vulnerability respond more readily to stressful life events with depressive feelings than individuals without a genetic vulnerability (Caspi et al., 2003). In this apparent interdependency between brain 5-HT, stress and depression, the hypothalamic-pituitary-adrenal (HPA) axis appears to play a major mediating role. In response to a physiological or psychological stressor, cell bodies at the level of the paraventricular nucleus of the hypothalamus are stimulated to release corticotropin-releasing hormone which, in turn, activates the anterior pituitary gland to secrete adrenocorticotropic hormone (ACTH). ACTH is secreted into the systemic circulation and stimulates the adrenal cortex to release glucocorticoid cortisol. Cortisol is the primary stress hormone, it re-establishes the internal balance of the nervous system and the body by exerting a variety of actions throughout the brain in order to terminate the stressresponse via negative feedback mechanisms, recover from stress and prepare the organism for stress coping (Sapolsky, 1992; Ursin \& Olff, 
1993; Dinan, 1994). There is ongoing evidence that the HPA system may be hyperactive in depression (e.g. De Kloet et al., 2005) and for complex inter-relationships between 5-HT and the HPA system (Arborelius et al., 1999; Holsboer, 2000; Van Praag, 2004; Firk \& Markus, 2007). Acute stress increases brain 5-HT turnover (e.g.De Kloet et al., 1982; De Kloet et al., 1983; Davis et al., 1995), which is important for the negative feedback control of cortisol on the HPA axis as a biological mechanism for stress adaptation (Nuller \& Ostroumova, 1980; Van Praag et al., 2004), whereas chronic stress decreases 5-HT turnover (Lopez et al., 1998; Fuchs \& Flugge, 2003), which may lead to an overactivation of the HPA axis and the onset of stress-related affective disorders (e.g. Markus, 2003; Van Praag et al. 2004). As a consequence, it has been suggested that chronically stressed subjects may benefit more from brain 5-HT augmentation, particularly under stress exposure, due to HPA axis and brain 5-HT interactions that have resulted in reduced 5-HT levels and subsequent 5-HT receptor sensitization as a compensatory response (Markus et al., 1998; Markus et al., 2000a; Markus, 2008). For instance, brain 5-HT augmentation by TRP-rich protein is found to reduce the negative effects of stress on cortisol stress-responses and depressive symptoms in healthy but stress-susceptible subjects compared to controls (Markus et al., 2000a; Markus et al., 2002). In accordance, Richell et al. (2005) reported that transient reduction of 5-HT concentrations through acute tryptophan depletion increases the negative effects of acute uncontrollable stress on mood.

Besides serotonergic vulnerability and stress, cognitive reactivity, a cognitive-psychological vulnerability marker of depression, is also implicated in depression (e.g. Teasdale, 1988; Van der Does, 2002; Lau et al., 2004). During unique experiences across life span, we develop selfreferent memory structures (schemas) that contain information about ourselves and our environment. Depending on the frequency and severity of undesirable incidences or experiences, cognitive schemes may become rather negative and dysfunctional with respect to self-efficacy and outcome-expectancies. The ease with which these dysfunctional cognitions appear during sad mood or negative circumstances is labeled 'cognitive reactivity' (CR). These cognitions are less active when the individual is in remission, but may become more activate when the person feels sad or experiences sad events (Teasdale, 1988). Previous research demonstrated that $\mathrm{CR}$ can predict relapse in recovered depressed pa- 
tients (Segal et al., 1999; Segal et al., 2006). Furthermore, CR is associated with an increased affective sensitivity to reductions of 5-HT concentrations in remitted patients; supporting relationships between CR and 5$\mathrm{HT}$ function and suggesting enhanced serotonergic vulnerability in subjects with high CR (Booij \& Van der Does, 2007).

Based on these findings, we suggest a relationship between $C R$, depression, 5-HT and stress. In more detail, stress particularly in subjects with high CR may increase or even induce serotonergic vulnerability, which in turn may lead to reduced 5-HT function, decreased stresscoping and an increased risk to develop depressive symptoms. As a consequence, and contrary to 5-HT depletion, brain 5-HT augmentation may have particular beneficial effects in subjects with high CR under stress; by counteracting diminished 5-HT function. Brain 5-HT concentrations can be augmented by increasing the availability of its precursor TRP (Young \& Gauthier, 1981). 5-HT is synthesized from the essential amino acid TRP which comes from protein in our diet. TRP is converted into 5-HTP (5hydroxytryptophan) by TRP-hydroxylase and then 5-HTP is converted into 5-HT by aromatic amino acid decarboxylase (AADC) (e.g. Fernstrom, 1990) Thus, the rate of 5-HT synthesis depends on TRP availability, which is the rate limiting step. Yet, TRP entry into the brain and 5-HT synthesis do not depend on the total amount of plasma TRP but are regulated by the relative availability of TRP versus the other large neutral amino acids (LNAA's) that compete with TRP for transport across the blood-brain barrier (Fernstrom \& Wurtman, 1971, 1972; Curzon, 1985). A promising method to increase TRP availability and thereby $5-\mathrm{HT}$ synthesis is by administration of alpha-lactalbumin, which is a whey-derived protein that contains more TRP than most proteins (Heine et al., 1996). It has been demonstrated that alpha-lactalbumin causes a $50-130 \%$ increase in plasma TRP:LNAA ratio (Markus et al., 2002; Markus et al., 2005; Merens et al., 2005; Booij et al., 2006), increases brain 5-HT (Orosco et al., 2004) and increases prolactin concentrations and has mood improving effects in chronically stressed subjects following acute stress exposure (Markus et al., 2000a). Nevertheless, the beneficial effects of alpha-lactalbumin on mood and stress-coping are only modest and do not always appear (Merens et al., 2005; Markus, 2008) suggesting that the increase in TRP:LNAA ratio may not be sufficient to affect behaviour. Recently, a TRP-rich hydrolyzed protein was tested with a TRP content and a 
TRP:LNAA ratio superior to alpha-lactalbumin revealing a positive effect on mood in healthy subjects (see Chapter 5).

The aim of the present study was to investigate the effects of a TRPrich hydrolyzed protein (HP) on mood and stress coping in healthy subjects with high CR as compared to low CR. Assuming serotonergic vulnerability in subjects with high $C R$, these subjects may be more responsive to the beneficial effects of the TRP-rich hydrolyzed protein than subjects with low CR after acute stress exposure.

\section{METHODS}

\section{Participants}

Maastricht University students $(n=150)$ filled in a questionnaire package concerning personal details (health, smoking and drinking habits, caffeine consumption, weight and height, and use of psychoactive drugs), several questionnaires concerning psychopathology (mood disorders, family history of depression, medical complaints, etc) and the Leiden Index of Depression Sensitivity (LEIDS) (Van der Does, 2002), a measure of CR. From the subjects showing interest in taking part in the study, 18 subjects with a low CR score (LEIDS score < 24) and 20 subjects with a high CR score (LEIDS score $>47$ ) were selected for the experiment. The low CR group (LCR) and the high CR group (HCR) did not differ with respect to sex, age and BMI scores (all $p>$.05). Demographic characteristics are presented in Table 1.

Participants were excluded if they reported chronic and current illness; history of psychiatric or medical illness; medication use; metabolic-, hormonal- or intestinal diseases; irregular diets; or deviant eating habits and excessive alcohol or drug use. Participants' health was checked with standardized medical questionnaires that were evaluated by a trained doctoral-level psychologist under the supervision of a medical doctor. Participants included in the study revealed normal body-mass indexes (BMI in $\mathrm{kg} / \mathrm{m}^{2}$ between 20 and 25), and were requested not to use alcohol or any kind of drugs before and during the study. All subjects had to fast overnight, only water and tea without sugar was permitted. The study was approved by the Ethic Committee Psychology (ECP) of Maastricht University and the procedures followed were in accordance with 
the Helsinki Declaration of 1975 as revised in 1983. All subjects gave their signed informed consent to participate in the experiment and received a financial compensation for their participation.

Table 1. Demographic characteristics. Values represent mean (SD).

\begin{tabular}{lll}
\hline & \multicolumn{2}{l}{ Cognitive Reactivity } \\
\cline { 2 - 3 } & high & low \\
\hline Women & 15 & 13 \\
Men & 5 & 5 \\
Age & $21.7(1.9)$ & $21.1(2.1)$ \\
BMI & $21.3(1.7)$ & $21.8(2.1)$ \\
LEIDS & $55.5(7.3)$ & $15.8(5.0)$ \\
\hline
\end{tabular}

\section{Design}

A placebo-controlled, double-blind, crossover design was used. During two experimental morning sessions, participants' mood and cortisol was assessed before and after acute stress exposure either following intake of HP or placebo (PLC). The order of presentation of the HP and PLC condition was counterbalanced within groups and both experimental sessions were separated by at least one week.

\section{Procedure}

On each experimental morning, participants arrived for testing at respectively 08:30 a.m., 09:00 a.m., 09:30 a.m. and 10:00 a.m. Every subject was invited to two experimental morning sessions. After arrival, a first baseline mood measurement was taken which was followed by the administration of the protein drink (HP or PLC). The order of presentation of the two different diets was counterbalanced between subjects. Between intake of the amino acid mixture and exposure to laboratory tasks, the subjects were able to study or to read magazines in a separated private room. They had free access to water and decaffeinated tea.

At baseline, participants' mood was assessed followed by the administration of the protein drinks which had to be ingested within 15 minutes. Two hours after intake of the protein drinks, a pre-stress cortisol sample $\left(t_{0}\right)$ was taken followed by a second mood assessment. Then, par- 
ticipants were exposed to the Trier Social Stress Test (TSST), again followed by mood assessment and acquisition of two post-stress cortisol samples (immediately after the TSST $\left(t_{+20}\right)$ and following a 10 minutes relaxation period $\left.\left(t_{+30}\right)\right)$ to measure stress-related activation of the HPA axis.

\section{Diets}

On both experimental morning sessions, a $312 \mathrm{ml}$ drink was consumed containing different tryptophan (TRP) or LNAA concentrations. The PLC condition included $0.4 \mathrm{~g}$ TRP and $10 \mathrm{~g}$ LNAA (DSM Delft; The Netherlands), whereas the HP condition (DSM Delft; The Netherlands) contained $0.8 \mathrm{~g}$ TRP and $2.7 \mathrm{~g}$ LNAA. The protein drinks were prepared by mixing the powder with $0.10 \mathrm{~g}$ sweetener (acesulfame) and were filled up by plane water in order to reach a $312 \mathrm{~mL}$ drink. Research assistants blind to the dietary conditions conducted the administration of the protein drinks.

\section{Subjective mood ratings}

Profile of Mood States (POMS). Changes in mood were measured using the Dutch shortened version of the POMS questionnaire (Wald \& Mellenbergh, 1990) offered as a visual analogue scale (VAS) ranging from 'strongly disagree' to 'strongly agree'. The POMS comprises five different subscales for mood; ranging from Anger, Depression, Fatigue and Tension that refer to a negative mood state, to Vigor concerning a positive mood.

Positive and Negative Affect Scale (PANAS). The PANAS (Watson et al., 1988) consists of two 10 items mood scales, a positive mood scale and a negative mood scale. The 20 items are randomly presented and participants have to respond on a scale ranging from 1 (totally disagree) to 5 (totally agree). 


\section{Trier Social Stress Test}

Psychosocial stress was induced with the Trier Social Stress Test (TSST) (Kirschbaum et al., 1993), which is a laboratory paradigm that reliably elicits physiological and psychological stress (Kirschbaum et al., 1993; Kirschbaum et al., 1999; Kudielka et al., 2004). It consists of a preparation period $(10 \mathrm{~min})$ followed by a video-taped test period (10 $\mathrm{min}$ ) during which the subject has to give a free speech $(5 \mathrm{~min})$ and perform mental arithmetic's (5 min) in front of an audience.

\section{Measurement of salivary cortisol}

Cortisol samples were obtained by using the Salivette sampling device (Sarstedt ${ }^{\circledR}$, Etten-Leur; the Netherlands). With this procedure, saliva was collected in small cotton swabs and stored $\left(-25^{\circ}\right.$ Celsius) immediately upon collection until centrifugation. Saliva samples were centrifuged at $2650 \mathrm{~g}_{\max }$ for three minutes at $20^{\circ} \mathrm{C}$. Salivary free cortisol levels was determined in duplicate by direct radioimmunoassay (RIA; University of Liège, Belgium), including a competition reaction between ${ }^{125}$ iodohistamine-cortisol and anti-cortisol serum made against the 3 CMO-BSA conjugate. After overnight incubation at $4^{\circ}$ Celsius of $100 \mu \mathrm{l}$ of saliva, separation of free and antibody-bound ${ }^{125}$ iodohistaminecortisol was performed via a conventional 'second antibody' method. In order to reduce sources of variability, all samples were analyzed in the same assay.

\section{Statistical analyses}

The main research questions were analyzed by means of repeated measures multivariate and univariate analyses of variance (MANOVA and ANOVA) by using the General Linear Model (GLM: SPSS 12.0 for Windows). Analyses were conducted with $C R$ (HCR vs. LCR) as betweensubjects factor and Treatment (HP vs. PLC) and Time (baseline vs. prestress) or Stress (pre-stress vs. post-stress) as within-subjects factors on mood and salivary cortisol concentrations. Although we already counterbalanced for Order of Treatment, Order and Gender were taken as centered covariates (Van Breukelen \& Van Dijk, 2007). All statistics are 
evaluated at a two-tailed significance level of 5\%. Greenhouse-Geisser corrected $\mathrm{P}$ values are reported when the sphericity assumption is not met. Post hoc analyses for significant interactions were assessed by paired and unpaired t tests to aid interpretation.

\section{RESULTS}

\section{Subjective mood ratings}

We conducted two repeated measures ANOVA with CR (HCR vs. LCR) as between-subjects factor and Treatment (HP vs. PLC) as withinsubjects factors for the subjective mood ratings. To investigate the effects of treatment and the effects of stress, separately, we included in the first analysis Time (baseline vs. pre-stress) and in the second analysis Stress (pre-stress vs. post-stress) as within-subjects factors. Data for the subjective mood rating scales are presented inTable 3. 
Table 3. Subjective mood ratings. Values represent mean (SD).

\begin{tabular}{|c|c|c|c|c|c|c|c|}
\hline \multirow[b]{3}{*}{ Test } & \multirow[b]{3}{*}{ Measure } & \multicolumn{6}{|c|}{ High Cognitive Reactivity } \\
\hline & & \multicolumn{3}{|l|}{ PLC } & \multicolumn{3}{|l|}{$\mathrm{HP}$} \\
\hline & & Baseline & Pre-stress & Post-stress & Baseline & Pre-stress & Post-stress \\
\hline \multicolumn{8}{|c|}{ PANAS } \\
\hline & Positive & $25.6(7.2)$ & $24.5(7.6)$ & $23.3(7.8)$ & $25.3(7.5)$ & $23.8(8.2)$ & $23.1(8.8)$ \\
\hline & Negative & $11.2(1.3)$ & $11.4(2.0)$ & $12.7(2.9)$ & $12.3(4.4)$ & $11.5(2.8)$ & $12.9(3.7)$ \\
\hline \multicolumn{8}{|c|}{ POMS } \\
\hline & Anger & $50.5(9.0)$ & $50.7(9.4)$ & $48.4(13.1)$ & $50.2(9.7)$ & $49.2(9.8)$ & $48.7(10.5)$ \\
\hline & Depression & $60.1(9.1)$ & $59.7(9.3)$ & $57.8(10.8)$ & $59.6(7.6)$ & $60.8(11.6)$ & $58.3(11.3)$ \\
\hline & Fatigue & $37.7(9.2)$ & $38.8(9.5)$ & $37.7(10.4)$ & $35.8(9.1)$ & $36.1(8.3)$ & $38.7(8.7)$ \\
\hline & Tension & $44.1(9.2)$ & $44.2(10.5)$ & $41.2(12.3)$ & $43.1(7.3)$ & $45.0(9.8)$ & $40.6(11.6)$ \\
\hline & Vigor & $16.1(7.6)$ & $14.9(6.8)$ & $14.8(6.2)$ & $14.9(6.5)$ & $16.2(8.0)$ & $15.6(6.9)$ \\
\hline \multirow[b]{3}{*}{ Test } & \multirow[b]{3}{*}{ Measure } & \multicolumn{6}{|c|}{ Low Cognitive Reactivity } \\
\hline & & \multicolumn{3}{|l|}{ PLC } & \multicolumn{3}{|l|}{ HP } \\
\hline & & Baseline & Pre-stress & Post-stress & Baseline & Pre-stress & Post-stress \\
\hline \multicolumn{8}{|c|}{ PANAS } \\
\hline & Positive & $23.9(5.7)$ & $21.9(6.3)$ & $21.6(6.1)$ & $24.8(8.0)$ & $22.4(6.5)$ & $23.3(5.9)$ \\
\hline & Negative & $15.7(4.9)$ & $13.6(4.1)$ & $14.5(4.8)$ & $15.0(3.9)$ & $15.0(4.03)$ & $15.4(5.3)$ \\
\hline \multicolumn{8}{|c|}{ POMS } \\
\hline & Anger & $45.0(7.5)$ & $45.2(9.5)$ & $43.3(12.5)$ & $43.4(12.1)$ & $44.4(11.7)$ & $42.5(11.8)$ \\
\hline & Depression & $52.9(9.1)$ & $51.8(11.6)$ & $52.1(14.1)$ & $50.5(10.1)$ & $50.3(12.6)$ & $51.5(12.3)$ \\
\hline & Fatigue & $33.3(10.4)$ & $32.8(12.2)$ & $33.4(12.8)$ & $27.9(10.6)$ & $30.3(11.9)$ & $33.9(11.0)$ \\
\hline & Tension & $39.8(9.9)$ & 41.5 (12.4) & $37.3(12.6)$ & $38.6(9.8)$ & $28.9(10.9)$ & $37.8(11.7)$ \\
\hline & Vigor & $20.0(7.5)$ & $18.6(7.0)$ & $19.2(8.7)$ & $21.2(9.1)$ & $23.1(8.7)$ & $19.8(6.9)$ \\
\hline
\end{tabular}

POMS. In the first analysis, MANOVA revealed a Treatment by Time interaction $[F(5,30)=2.96, p=.027]$. Univariate analysis revealed that the effect originated from the subscale vigor $[F(1,34)=8.37, p=.007]$ showing an increase in positive mood following $\mathrm{HP}[t(37)=2.1, p=.04]$ but not $\operatorname{PLC}[t(37)=1.49, p=.15]$. The interaction is visualized in Figure 1.

In the second analysis, MANOVA only showed a main effect of Stress $[F(5,30)=3.93, p=.007]$ originating from the subscale tension $[F(1,34)=11.14, p=.002]$ reflecting lower tension scores post-stress than pre-stress.

PANAS. In the first analysis, we found a main effect of Time $[F(1,34)=11.87, p=.002]$ on the positive scale of the PANAS reflecting 
an overall slight decrease in positive mood across time. No effects were found for the negative scale of the PANAS.

In the second analysis, we found no effect for the positive scale of the PANAS but a marginal significant main effect of stress $[F(1,34)=4.09$, $p=.051]$ on the negative scale of the PANAS revealing a slight increase in negative mood post-stress compared to pre-stress.

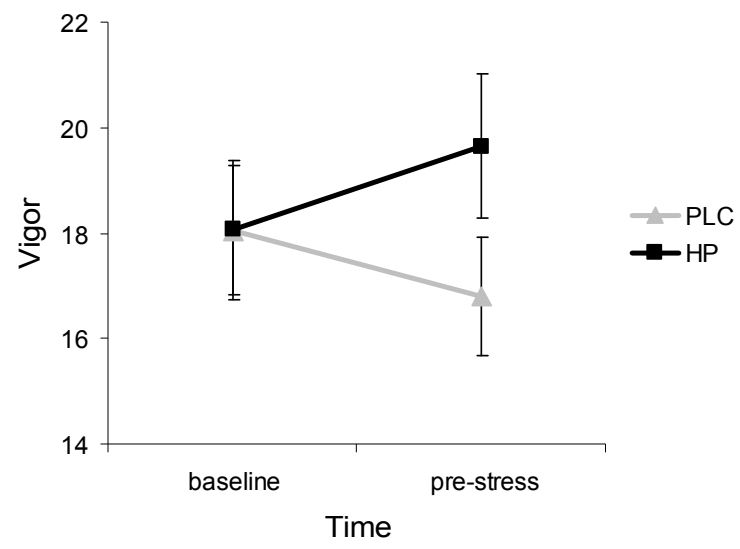

Figure 1. Mood was improved following intake of HP as measured by higher scores on the vigor scale of the POMS.

\section{Cortisol}

Repeated measures analysis of variance with $C R$ (HCR vs. LCR) as between-subjects factor and Treatment (HP vs. PLC) and Stress $\left(t_{0}\right.$ vs. $t_{+20}$ vs. $\left.t_{+30}\right)$ as within-subjects factors on salivary cortisol concentrations revealed a main effect of Stress $[F(2,33)=6.86, p=.003]$, reflecting an increase in cortisol following the TSST, and a main effect of Treatment $[F(1,34)=10.15, p=.003]$ indicating lower cortisol concentrations following HP than PLC. Further, we found a marginal significant Treatment $X$ Stress interaction $[F(2,68)=6.53, p=.067]$ reflecting no significant difference pre-stress $[t(37)=1.8, p=.08]$ but higher cortisol concentrations post-stress following PLC than HP $\left[t_{+20}: t(37)=2.7, p=.01 ; t_{+30}: t(37)=2.8\right.$, $p=.008$ ] (figure 2). Since the cortisol concentrations were highest 30 minutes following stress onset $\left(t_{+30}\right)$, we conducted a second analysis including the 2-level factor Stress with the pre-stress measure $\left(t_{0}\right)$ and the second post-stress measure $\left(t_{+30}\right)$ as within subjects factor. Analysis re- 
vealed a significant Treatment $X$ Stress interaction $[F(1,34)=4.34, p=$ .045], reflecting a dampened cortisol response following HP compared to PLC $[t(37)=2.2, p=.037]$ (figure 2). Cortisol data can be found in table 4.

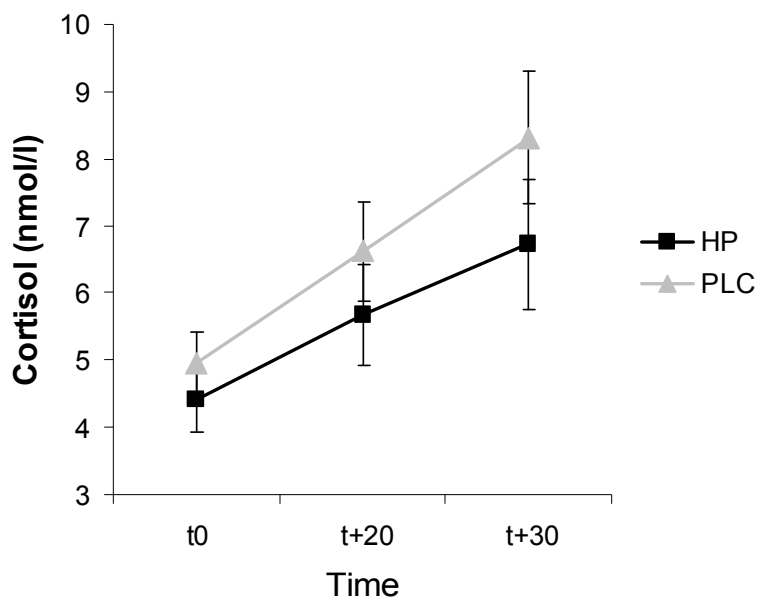

Figure 2. No significant difference between treatments in cortisol concentrations pre-stress were found [ $t 0: t(37)=1.8, p=.08$ ] but higher cortisol concentrations post-stress following PLC than HP $\left[t_{+20}: t(37)=2.7, p=.01 ; t_{+30}: t(37)=2.8, p=.008\right]$ reflecting a dampened cortisol response following HP compared to PLC.

Table 4. Cortisol data (nmol/l). Values represent mean (SD).

\begin{tabular}{cllll}
\hline & \multicolumn{2}{l}{ Low Cognitive Reactivity } & \multicolumn{2}{l}{ High Cognitive Reactivity } \\
\cline { 2 - 5 } & PLC & HP & PLC & HP \\
\hline Time & $4.8(2.3)$ & $4.5(2.8)$ & $4.8(2.3)$ & $4.3(3.1)$ \\
t0 (pre-stress) & $6.8(4.6)$ & $5.5(4.6)$ & $6.3(4.2)$ & $5.8(4.5)$ \\
t+20 (post-stress) & $8.1(5.8)$ & $6.7(5.7)$ & $8.3(6.4)$ & $6.8(6.2)$ \\
t+30 (post-stress) & & &
\end{tabular}

\section{DISCUSSION}

The aim of the present study was to investigate the effects of a TRPrich hydrolyzed protein (HP) intake on mood and stress coping in healthy subjects and to examine whether subjects with high cognitive reactivity (CR) are more responsive to the beneficial effects of HP than subjects with low $C R$. 
Previous research including TRP-rich intact alpha-lactalbumin protein already indicated consistent significant $70-130 \%$ increases in the plasma TRP:LNAA ratio (Markus et al., 2002; Markus et al., 2005; Merens et al., 2005; Booij et al., 2006). Recently, a hydrolyzed and more purified TRP-rich protein was found to cause a much greater increase in plasma TRP:LNAA; largely exceeding the effects of alpha-lactalbumin protein (Markus et al., 2008). It has been suggested that a $40-70 \%$ variation in plasma TRP:LNAA could be sufficient to change brain $5-\mathrm{HT}$ synthesis in animals as well as humans; either reducing brain $5-\mathrm{HT}$ by lowering the TRP:LNAA ratio through ATD (Biggio et al., 1974; Nishizawa et al., 1997; Carpenter et al., 1998; Williams et al., 1999) or increasing 5-HT by enhancing the TRP:LNAA ratio trough TRP augmentation (Fernstrom \& Wurtman, 1972; Markus et al., 2000a; Orosco et al., 2004). Since the recently used TRP-rich hydrolyzed protein largely exceeded this threshold (Markus et al., 2008), it is very likely that also the current HP causes a much greater rise in 5-HT availability and consequently also has a stronger effect on behavior and affect.

In line with our previous findings (see Chapter 5), we found an increase in positive mood following HP, which we assume is caused by a substantial increase in TRP:LNAA ratio and subsequent increased brain 5-HT function. In support, 5-HT synthesis is positively correlated with positive mood and negatively correlated with negative mood (PerreauLinck et al., 2007) and whole blood 5-HT concentrations have been found to predict mood in healthy individuals (Williams et al., 2006). Thus, the beneficial effects of HP on mood in healthy subjects suggest that the substantial increase in TRP:LNAA and 5-HT synthesis is sufficient to affect behaviour also in non-clinical populations in contrast to previously used TRP-rich proteins such as alpha-lactalbumin whey-protein that only affected mood after acute stress exposure in chronically stressed subjects (Markus et al., 2000a).

Further, we explored the beneficial effects of HP on stress coping. Following acute stress exposure, we found a slight increase in negative mood and a clear-cut cortisol response > than $2.5 \mathrm{nmol} / \mathrm{l}$. This resembles earlier findings reporting psychological and physiological stress following the TSST (Kirschbaum et al., 1993; Kirschbaum et al., 1999; Smeets et al., 2006). Furthermore, we found a treatment by stress interaction revealing a dampened cortisol response $(<2.5 \mathrm{nmol} / \mathrm{l})$ following HP compared to PLC regardless of CR, which may be attributed to en- 
hanced stress resilience due to HP. This may be caused by increased 5HT synthesis supporting the proposal that dietary-induced increases in TRP:LNAA ratio have beneficial effects on stress coping and is in line with evidence of 5-HT involvement in stress coping (Porter et al., 2004). Acute stress increases 5-HT neurotransmission (De Kloet et al., 1982; De Kloet et al., 1983; Davis et al., 1995), which promotes stress adaptation by mediating negative feedback control of cortisol on the HPA axis (Nuller \& Ostroumova, 1980; Van Praag, 2004). Deakin and Graeff (1991) suggested that particularly the 5-HT pathway originating from the median raphe nucleus (MRN) and projecting to the dorsal hippocampus may mediate resilience to repeated stress. In line with our findings, Richell et al. (2005) reported lower mood following acute uncontrollable stress after ATD, which decreases the TRP:LNAA ratio and reduces 5-HT synthesis (Fernstrom \& Wurtman, 1972; Young et al., 1985). In further support, dietary-induced $50-70 \%$ increases in TRP:LNAA ratio by alphalactalbumin have been found to reduce cortisol responses and lower mood in chronically-stressed subjects following an uncontrollable mental arithmetic stress task (Markus et al., 2000a; Markus et al., 2002). It was hypothesized that increases in TRP:LNAA ratio and 5-HT synthesis only positively affected chronically stressed subjects due to stress-induced receptor sensitization of their brain 5-HT system. In the current study, however, dietary 5-HT augmentation by the use of a hydrolyzed protein with a superior effect on the TRP:LNAA ratio than intact alphalactalbumin and pureTRP (Markus et al., 2008) also dampened the physiological stress response in healthy non-vulnerable subjects supporting the assumption that the use of HP may be a more adequate method to further augment brain TRP and 5-HT at a more meaningful level and to improve 5-HT-related stress resilience (Markus et al., 2008).

Our second aim was to explore whether subjects with high CR are more responsive to the beneficial effects of HP than subjects with low $C R$. As already mentioned in the introduction, $C R$ is the ease with which dysfunctional cognitions appear during sad mood and previous studies have shown that CR predicts depressive relapse (Segal et al., 1999; Segal et al., 2006) and serotonergic vulnerability (Booij \& Van der Does, 2007). Contrary to our expectation that HP may be more effective in individuals with high $C R$ due to serotonergic vulnerability, CR did not mediate the beneficial effects of HP in the current study. It should be noted that the association between $\mathrm{CR}$ and serotonergic vulnerability has been 
found in an ATD study with depressive patients in remission (Booij \& Van der Does, 2007), which may explain our negative findings in healthy nonvulnerable subjects. Furthermore, ATD induces depressive relapse by transiently decreasing 5-HT synthesis, whereas HP improves mood by enhancing $5-\mathrm{HT}$ synthesis. CR, as assessed by the LEIDS, predicts access to dysfunctional schemas during low mood, which is thought to represent a trait marker for depression vulnerability (Booij \& Van der Does, 2007). Therefore, we suggest that individuals with high CR may benefit from HP to a greater extent during sad mood, which may be explored in future studies.

In conclusion, HP positively affects mood and stress coping in healthy non-vulnerable subjects. In future studies, clinical or sub-clinical individuals should be included to further explore the beneficial effects of $5-\mathrm{HT}$ augmentation in these populations and to determine whether this dietary manipulation may have therapeutic potential in $5-\mathrm{HT}$ related disorders such as depression. 


\section{CHAPTER 7}

General Discussion 


\section{AIM OF THE DISSERTATION}

The main aim of this dissertation was to investigate the interaction between stress and brain serotonergic vulnerability as a risk factor for depression. Serotonergic vulnerability has been defined as sensitivity of the serotonergic system to alterations or disruptions, which increases the likelihood of developing disorders that are related to the serotonergic system, such as depression (Jans et al., 2007). Serotonergic vulnerability has been postulated for individuals with an increased risk for depression including individuals with a family history of depression $(\mathrm{FH}+)$ (Benkelfat et al., 1994; Klaassen et al., 1999), individuals with allelic variation in genes that encode transporter-proteins that are critical in determining the overall level of serotonergic neurotransmission (e.g. Neumeister et al., 2002), and individuals with a cognitive reactivity to depression (Booij \& Van der Does, 2007). However, a major problem is that serotonergic vulnerability is not the only factor involved in the development of depression. Many individuals with a serotonergic vulnerability do not develop depression, suggesting that the relationship between serotonin (5HT) dysfunction and depressive symptoms is not direct, but involves an additional factor. One factor that has been proposed to play an important role in the development of depression is stress. Depression is often preceded by stress and epidemiological studies suggest that serotonergic vulnerability predisposes individuals to depression only in the context of stress (e.g. Caspi et al., 2003). Further, it has been suggested that stress may potentiate serotonergic vulnerability and that a vulnerable serotonergic system and stress may mutually increase the risk of depression. In support of this assumption, interactions between the serotonergic system and the stress response have been clearly reported and challenges of either system may reduce the function of the other (e.g. Porter et al., 2004; Van Praag et al., 2004; Firk \& Markus, 2007). Nevertheless, the interaction between serotonergic vulnerability and stress has not yet been investigated experimentally in depression-vulnerable individuals despite extensive epidemiological studies that propose a link between serotonergic vulnerability, stress, and the development of depression. Therefore, in this research project, the interaction between serotonergic vulnerability, stress resilience, and depressive symptoms in individuals with increased risk for depression was investigated in several doubleblind placebo-controlled experimental designs. In these studies, depres- 
sion-prone subjects with assumed serotonergic vulnerabilities related to a positive family history of depression $(\mathrm{FH}+$ ), a low expressing 5-HTTLPR $s$ allele, or a cognitive reactivity (CR) to depression were compared with control participants for cognitive-affective (mood, memory, attention) and biological (cortisol, plasma amino acids) changes under laboratory stress either following acute tryptophan depletion (ATD), tryptophan (TRP) augmentation, or placebo. In the following sections, the main research findings are summarized and discussed, methodological questions are raised, and directions for future research are given.

\section{SUMMARY AND DISCUSSION OF THE FINDINGS}

As previously described, the main rationale of the present research project was to explore interactions between serotonergic vulnerability, stress, and depressive symptoms in individuals with an increased risk for depression. To summarize and interpret the findings, the following two questions will be addressed: 1) do depression-prone subjects (i.e., individuals with a family history of depression, with the s allele of the 5HTTLP, or with high CR to depression) indeed exhibit serotonergic vulnerability? and 2) do depression-prone individuals exhibit increased stress vulnerability, particularly following 5-HT depletion, and decreased stress vulnerability following 5 -HT augmentation?

\section{Serotonergic vulnerability}

Serotonergic vulnerability, or the sensitivity of the serotonergic system to alterations or disruptions, in depression-vulnerable individuals was examined by means of two different experimental manipulations, either depleting brain 5-HT by ATD or increasing brain 5-HT by dietary TRP augmentation. ATD has been shown to lower brain 5-HT synthesis and activity by depletion of its precursor TRP relative to the sum of the other large neutral amino acids (LNAAs) with which TRP competes for uptake into the brain (Fernstrom \& Wurtman, 1972; Young et al., 1985). This was accomplished through administration of a gelatin-based hydrolyzed collagen protein (CP) that contains the entire range of amino acids except for L-TRP (Evers et al., 2005; Sambeth et al., 2008). Previous stud- 
ies have clearly demonstrated that in humans, ATD successfully reduces brain 5-HT function by decreasing the plasma TRP:LNAA ratio (Nishizawa et al., 1997; Carpenter et al., 1998; Williams et al., 1999). In Chapters 2, 3 , and 4 it has been shown that the CP ATD method lowered the TRP:LNAA ratio by $65-77 \%$, which is comparable to previous studies using the same CP method (Evers et al., 2005; Sambeth et al., 2008) or the classic ATD mixture (Van der Does, 2001). In Chapter 2, it was found that individuals with $\mathrm{FH}+$ were slightly more vulnerable to ATD (longer RTs on the affective go/no-go (AGNG) task to happy words on non-shift blocks) than control participants, which is consistent with earlier findings reporting ATD vulnerability in $\mathrm{FH}+$ individuals (Benkelfat et al., 1994; Klaassen et al., 1999). In Chapter 4, ATD lowered mood in all subjects independent of genetic vulnerability but impaired immediate recall of positive words in genetic 5-HT-vulnerable S/S genotypes only. In addition, ATD increased the number of errors during happy shift blocks on the AGNG task only in 5-HT-vulnerable S/S genotypes. These findings seem to support the assumption of increased serotonergic vulnerability and clearly resembles the mood-congruent memory bias that has often been reported in depressive patients, reflecting impaired memory for positive information (Matt et al., 1992).

Brain 5-HT augmentation was accomplished through the use of a TRP-rich hydrolyzed protein that has been shown to increase the TRP:LNAA ratio (Chapter 5). This increase in TRP:LNAA ratio largely exceeds the effect of pure TRP (Chapter 5) and is much greater than that for previously used TRP-rich proteins (Markus et al., 2002; Markus et al., 2005; Merens et al., 2005; Booij et al., 2006). Furthermore and contrary to previously used intact TRP-rich proteins, the hydrolyzed TRP-rich protein increased positive mood in healthy non-vulnerable subjects (Chapters 5 and 6). Since 50-70\% increases in plasma TRP:LNAA are already found to be sufficient to increase brain 5-HT function (Markus et al., 2000a; Orosco et al., 2004), the beneficial effects of a hydrolyzed TRPrich protein on mood in healthy subjects are most likely due to increased 5-HT synthesis (Markus et al., 2008). Contrary to our expectation that TRP augmentation may be more effective in individuals with high CR due to serotonergic vulnerability, this mediating effect of $C R$ was not found. It should be noted that the association between $C R$ and serotonergic vulnerability has been suggested in an ATD study including depressive patients in remission (Booij \& Van der Does, 2007), which may explain 
our negative findings in healthy non-vulnerable subjects following dietary TRP augmentation. After all, TRP augmentation improves mood by enhancing 5-HT synthesis, whereas ATD lowers mood by transiently decreasing 5-HT synthesis and CR predicts access to dysfunctional schemas during low mood, a trait marker for depression vulnerability (Booij \& Van der Does, 2007). Hence, individuals with high CR may benefit from TRP augmentation to a greater extent during sad mood, which may be explored in future studies. Further, we suggest that future studies should also include clinical or sub-clinical populations to further examine the association between $\mathrm{CR}$ and serotonergic vulnerability and investigate the beneficial effects of 5-HT augmentation in these populations as, hypothetically, CR may be associated with serotonergic vulnerability only in depressive patients in remission. After all, previous studies have shown that $\mathrm{CR}$ can predict depressive relapse in these populations (Segal et al., 1999; Segal et al., 2006).

Based on these findings, it can be concluded that $\mathrm{FH}+$ individuals and individuals with the 5-HTTLPR s allele are slightly more vulnerable to affective consequences of serotonergic manipulations than healthy control participants. Yet, the effects appear to be small and should be interpreted with caution, particularly regarding clinical relevance. On the contrary, healthy individuals with high CR do not seem to benefit more from $5-\mathrm{HT}$ augmentation than healthy individuals with low $\mathrm{CR}$, suggesting that $\mathrm{CR}$ is not associated with serotonergic vulnerability as found in $\mathrm{FH}+$ subjects and 5-HTTLPR s allele carriers. However, CR, as compared to FH+ and S/S 5-HTTLPR, is more directly related to the ease with which dysfunctional thoughts are activated during sad mood. Therefore, serotonergic vulnerability in $\mathrm{CR}$ subjects may only become visible during low mood and may, therefore, be explored in future ATD studies.

\section{Serotonergic vulnerability, stress, and depression}

The major aim of the current studies was to explore whether depression-vulnerable individuals exhibit stress vulnerability and whether serotonergic vulnerability for depression may particularly manifest itself under stress and whether this is further affected by brain 5-HT manipulations. To investigate this interaction between stress, 5-HT vulnerability, and depression experimentally, physiological and psychological stress was induced in the current studies. This was achieved by means of two 
experimental stress procedures: the Markus-Peters computerized mental arithmetic task (MPA) (e.g. Markus et al., 1998) and the Trier Social Stress Test (TSST) (Kirschbaum et al., 1993). In Chapters 2, 3, and 4, the MPA task was used to induce physiological and psychological stress. In Chapter 2, reaction times (RTs) on the AGNG task significantly decreased after the stress task. In Chapter 4, stress increased negative mood scores, resulting in longer RTs on the AGNG task and lowered delayed recall. Although the MPA stress task affected mood and behavior, cortisol responses were not increased following stress exposure, suggesting that the MPA task was only a brief and mild stressor in the current designs (Chapter 4). In Chapter 6, the TSST was used to induce acute stress. Following the TSST, we found an increase in negative mood and a clearcut cortisol response $>2.5 \mathrm{nmol} / \mathrm{l}$, in line with earlier findings reporting psychological and physiological stress following the TSST (Kirschbaum et al., 1993; Kirschbaum et al., 1996; Smeets et al., 2006).

In Chapter 3, we found a genotype by stress by shift interaction on the AGNG task, indicating fewer omissions for targets on non-shift blocks post-stress compared to pre-stress for individuals with the $1 / 1$ genotype. In Chapter 4, we found a stress-induced increase in depression scores exclusively in s/s genotypes, whereas in $1 / /$ genotypes stress even increased positive feelings of vigor. These findings seem to support previous assumptions of 5-HTTLPR s allele as a vulnerability factor in the development of depression and suggest that I/I carriers are less susceptible to the negative effects of acute stress than those with s/s genotypes. As already discussed in the general introduction, 5-HT plays an important role in stress coping, which may explain why I allele carriers are more resistant to the negative effects of stress compared to $s$ allele carriers. This involvement of $5-\mathrm{HT}$ in stress coping is supported by complex interactions between 5-HT and the hypothalamic-pituitary-adrenal (HPA) stress response (Porter et al., 2004). Acute stress increases 5-HT neurotransmission (De Kloet et al., 1982; De Kloet et al., 1983; Davis et al., 1995), which promotes stress adaptation by mediating negative feedback control of cortisol on the HPA axis (Nuller \& Ostroumova, 1980; Van Praag, 2004). In compliance with the current findings, 5HTTLPR s allele carriers are at increased risk of depression, particularly in the face of stress (e.g. Caspi et al., 2003). In further support, Gotlib (2008) found that only girls homozygous for the s allele showed a cortisol 
response following acute stress exposure, which also supports the hypothesis that 5-HTTLPR affects HPA activity and, hence, stress reactivity.

In Chapter 2, individuals without a family history of depression showed stress-induced slowed responses to happy words in non-shift blocks on the AGNG task only following ATD, whereas the FH+ group showed this pattern after ATD (pre- and post-stress) as well as after stress following PLC. These findings suggest that FH- subjects may be more stress-resilient than $\mathrm{FH}+$ subjects and may become susceptible to stress only after ATD. This may be attributed to enhanced stress susceptibility caused by ATD-related (transient) dysfunction of the 5-HTmediated resilience system (Richell et al., 2005). Deakin and Graeff (1991) suggested that the 5-HT pathway originating from the median raphe nucleus (MRN) and projecting to the dorsal hippocampus may particularly mediate resilience to repeated stress. Psychosocial stress may, therefore, predispose individuals to depression mainly by attenuating 5$H T_{1 A}$ receptor functioning in the MRN resilience system via stress hormone mechanisms. Although acute psychosocial stress increases hippocampal 5-HT concentrations, as a biological condition for stress adaptation, chronic stress is found to downregulate $5-\mathrm{HT}_{1 \mathrm{~A}}$ hippocampal receptors in animals (Lopez et al., 1998; Fuchs \& Flugge, 2003). Consistently, $5-\mathrm{HT}$ challenge in chronically-stressed subjects, but not in controls, increased prolactin responses and improved mood and performance after acute stress (Markus et al., 2000a; Markus et al., 2002), and depressed patients show decreased $5-\mathrm{HT}_{1 \mathrm{~A}}$ receptor binding (Drevets et al., 1999; Sargent et al., 2000) and reduced hippocampal volume (Sheline et al., 2003) .

In support of the serotonergic vulnerability by stress interaction hypothesis, we found a dampened cortisol response to an acute stressor (TSST) following TRP augmentation (Chapter 6), which we suggest may be attributed to enhanced stress resilience due to increased 5-HT availabilty. This supports the proposal that dietary-induced increases in TRP:LNAA ratio have beneficial effects on stress coping (Markus, 2008) and is in line with the 5-HT-mediated resilience system (Richell et al., 2005).

In sum, the present findings support the role of stress as an important predecessor in the development of depression. Moreover, negative effects of stress on depression-related cognitive-affective changes were found to depend on FH and 5-HTTLPR genotypes. Individuals with $\mathrm{FH}+$ 
were found to be more susceptible to the negative affective effects of stress than $\mathrm{FH}$ - individuals. Furthermore, individuals homozygous for the $s$ allele of the 5-HTTLPR show higher depression scores after stress, whereas in individuals homozygous for the I allele, stress even increased positive mood. In further support, 5-HT augmentation enhanced stressresilience in line with evidence of 5-HT involvement in stress coping. These findings clearly support the assumed interaction between serotonergic vulnerability, stress, and depression. Nevertheless, these interaction effects appeared to be small, which also raises methodological questions regarding the experimental manipulations.

\section{METHODOLOGICAL ISSUES}

Although the findings summarized and discussed above support the interaction between serotonergic vulnerability, stress, and depression, effects were rather modest, which may be related to the experimental manipulations (i.e., 5-HT manipulations and stress manipulations). The current findings suggest that brain 5-HT manipulations (i.e., brain 5-HT depletion (through ATD) and brain 5-HT augmentation (through TRP augmentation)) have been sufficiently conducted. After all, previous studies have shown that a $40-70 \%$ variation in plasma TRP:LNAA is sufficient to change brain 5-HT synthesis and function in animals as well as humans (Fernstrom \& Wurtman, 1972; Biggio et al., 1974; Nishizawa et al., 1997; Carpenter et al., 1998; Williams et al., 1999; Markus et al., 2000a; Orosco et al., 2004). Since ATD resulted in a 65-77\% decrease in TRP:LNAA ratio (Chapters 2, 3, and 5) and TRP augmentation resulted in a $255 \%$ increase in TRP:LNAA ratio (Chapter 5), it seems to be safe to conclude that both manipulations were successful in causing a sufficient increase or decrease in brain TRP and 5-HT and, subsequently, to have an effect on cognitive-affective behavior.

On the contrary, the current stress manipulations may have been too mild to find strong evidence for interactions between serotonergic vulnerability, stress, and depression in depression-vulnerable subjects. To explore these interactions, laboratory stress tasks were used that were thought to activate the HPA stress response. Based on previous studies showing successful stress initiation by the MPA task and the TSST, in the present studies these tasks were included as acute stress manipulations (e.g. Kirschbaum et al., 1993; Markus et al., 1998; Markus et al., 2000a). 
In the current experiments, both stress tests affected mood but a cortisol response was only found in reaction to the TSST. According to Lupien (2007), stress can be relative or absolute in nature. Absolute stress is a real threat that elicits a stress response in every person (e.g., earthquake), whereas relative stress is an implied threat that is induced by the interpretation of a situation as stressful. Therefore, giving a speech in front of an audience, as during the TSST, or performing unsolvable mental arithmetic problems, as during the MPA task, may be very stressful for some individuals but not for all individuals. In accord, large interindividual variations in the stress response to relative stress situations have been reported (e.g. Kirschbaum et al., 1999; Kudielka et al., 2004; Smeets et al., 2006). Lupien (2007) argued that absolute stressors, due to their life-threatening nature, will automatically lead to a physiological response, whereas relative stressors require a cognitive interpretation in order to elicit a stress response. Therefore, the cognitive evaluation of a given situation is responsible for the activation of the stress response. Psychological factors that influence cognitive stress perception are novelty, unpredictability, and uncontrollability (Mason, 1968). The MPA as well as the TSST have both uncontrollable and unpredictable components; however, the repeated measures design may decrease feelings of novelty, uncontrollability, and unpredictability, which may, in turn, reduce cognitive stress evaluation. According to Dickerson and Kemeny (2004), social evaluative threat (i.e., the threat that task performance could be negatively evaluated by others) also activates the HPA system based on the self-preservation theory assumption that individuals monitor their environment for threats to their social esteem or status. Therefore, in Chapter 4, to increase social evaluative threat, the MPA task was performed under supervision of the experimenter. Nonetheless, this was still insufficient to activate the HPA axis and we suggest that, in future studies, particularly in repeated measures design, the TSST should be used instead.

In the general introduction, it has been proposed that stress in individuals with a serotonergic vulnerability may induce depressive symptoms. In the present research project, we modelled this by inducing serotonergic vulnerability through ATD and acute stress by means of the MPA. It was hypothesized that following ATD, 5-HT does not increase under acute stress (because 5-HT synthesis is dependent on TRP availability), causing ATD-related (transient) dysfunction of the 5-HT- 
mediated resilience system. Therefore, we expected that participants may have been more vulnerable to stress following ATD. This has also been supported by lowered mood in healthy subjects following ATD and uncontrollable stress (Richell et al., 2005) and by increased cardiovascular and psychological stress responses following ATD in recovered patients with anxiety disorders (Davies, 2006). In the present research project, interactions between ATD and stress were only rarely found, which may be related to the mild acute nature of the stressors. In the ATD studies, the MPA stress task (likely) did not activate the HPA axis as indicated by the missing cortisol response (Chapter 4). However, it was expected that decreased 5-HT synthesis would negatively effect stress coping particularly due to the fact that $5-\mathrm{HT}$ plays an important role in regulating HPA axis activity and supporting the negative feedback control of cortisol on the HPA axis (Porter et al., 2004). Therefore, the interaction between ATD and acute stress in 5-HT-vulnerable individuals should be investigated in future studies including a more severe stress task. Yet, it may be questioned whether acute stress is a good instrument to model every-day life stress or stressful life events. After all, epidemiological studies that proposed an interaction between serotonergic vulnerability, stress, and depression concentrated on stressful life events. Further, it is very likely that stressful life events are more intense than an acute laboratory stressor. Pre-clinical and clinical studies suggest that, in particular, early stressful life events have long-lasting effects on the HPA axis as implied by chronic increases in glucocorticoids and ACTH levels and enhanced neuroendocrine response to stressors in adulthood that are possibly due to CRH overdrive (see for reviews Heim \& Nemeroff, 2001; Heim et al., 2008). In support, a recent experimental study has found that the combination of early life stress and chronic stress during adolescence was the best predictor of cortisol response to an acute stressor (Rao et al., 2008). Therefore, future research projects may also focus on the development of experimental designs investigating the interaction between (early) stressful life events and/or chronic stress, serotonergic vulnerability, and depression.

\section{CONCLUSION}

The central focus of this research project was to investigate the interaction between serotonergic vulnerability, stress, and depressive symp- 
toms in individuals with increased risk for depression in several doubleblind placebo-controlled experimental designs. The first main question of the present studies was to explore whether subjects with a family history of depression, subjects with the s allele of the 5-HTTLP, and subjects with a high cognitive reactivity to depression, who were considered to have an increased risk of developing depression due to a 5-HT vulnerable system, indeed, show evidence of serotonergic vulnerability. The second aim of the current studies was to determine whether depression(5-HT-) vulnerable individuals are prone to the negative effects of acute stress, particularly following depleting $5-\mathrm{HT}$ through ATD, and whether negative effects of stress can be counteracted by increasing 5-HT availability through TRP augmentation.

In sum, results from the present project revealed that $\mathrm{FH}+$ individuals and individuals with the 5-HTTLPR s allele are slightly more vulnerable to serotonergic manipulations than healthy non-vulnerable control participants. Negative effects of stress may depend on family history of depression and 5-HTTLPR genotype, supporting the interaction between serotonergic vulnerability, stress, and depression. In support, dietaryinduced increases in TRP:LNAA ratio through a hydrolyzed TRP-rich protein enhanced stress coping and mood in healthy subjects.

Future research projects may also focus on the development of experimental designs investigating the interaction between (early) stressful life events, serotonergic vulnerability, and depression. After all, early life stress seems to have long-lasting effects on the HPA axis and is associated with enhanced adrenal reactivity to acute psychosocial stress. Further, future research is needed to explore whether TRP augmentation through a hydrolyzed TRP-rich protein may even be more relevant in affected or vulnerable subjects and may have therapeutic potential in 5HT-related disorders.

Taken together, the present studies were the first to experimentally investigate the interaction between serotonergic vulnerability and stress in depression-vulnerable (5-HT-vulnerable) individuals. The information provided in this dissertation contributes to the understanding of early risk factors for depression, suggests interactions between biological and psychological factors that are indicated in determining vulnerability to stress and depression, and, further, presents promising ideas for future research. 

SUMMARY 
Affective disorders such as depression are one of the most common disease burdens throughout the world and are associated with severe medical consequences and mortality. Finding risk factors for depression is, therefore, a major challenge today. Among the different factors involved, a genetic predisposition appears to be a potential risk factor for the development of depression. Although the exact neurobiological equivalent of this genetic predisposition remains unclear, it seems as though the brain serotonin (5-HT) system plays an important mediating role. Evidence suggests that individuals with a serotonergic vulnerability, which has been defined as vulnerability or sensitivity of the serotonergic system to alterations or disruptions, have an increased risk of developing affective disorders that are related to the serotonergic system, such as depression (Jans et al., 2007). Serotonergic vulnerability has been postulated for individuals with a family history of depression $(\mathrm{FH}+)$ (Benkelfat et al., 1994; Klaassen et al., 1999) and for individuals with allelic variation in genes that encode 5-HT transporter proteins (5-HTTLPR polymorphism) that are critical in determining the overall level of serotonergic neurotransmission (e.g. Neumeister et al., 2002). A major problem, however, is that the role of brain 5-HT in depression is complex and that a 5HT-related innate vulnerability, by itself, does not appear to be sufficient enough to cause a depressive episode. In the search for additional factors, stress has received particular attention. Stressful life events influence and precede the onset of depression. Furthermore, depression is associated with stress hormone dysregulation and bi-directional interactions are thought to occur between stress-related changes in the neuroendocrine stress system and the 5-HT system. Even though a variety of impressive epidemiological studies support this intriguing assumed interaction between stress, serotonergic vulnerability, and depression, this interaction has not yet been investigated in experimental designs. Therefore, the aim of this dissertation was to experimentally investigate the interaction between stress and serotonergic vulnerability as a risk factor for depression.

Different population groups that are suggested to have an increased risk of depression due to serotonergic vulnerability (i.e., those with a family history of depression, 5-HTTLPR s allele carriers, and individuals with a cognitive vulnerability to depression) were exposed to serotonergic manipulations (i.e., either increasing or reducing brain 5-HT) and acute stress to explore the interaction between serotonergic vulnerabil- 
ity, stress, and depressive symptoms. In Chapters 2, 3, and 4, brain 5HT synthesis and activity was lowered by acute tryptophan depletion (ATD), which reduces the amount of plasma tryptophan (TRP) relative to the sum of the other large neutral amino acids (LNAAs) with which TRP competes for uptake into the brain (Fernstrom \& Wurtman, 1972). This ATD was accomplished through administration of a balanced TRP-free amino acid mixture, which contains all essential amino acids except for TRP. This reduces the amount of plasma TRP as compared to the LNAAs (TRP:LNAA ratio) and, thus, increases competition of the LNAAs with which TRP competes for uptake into the brain (e.g. Gessa et al., 1974; Moja et al., 1991; Maes \& Meltzer, 1995). In Chapters 5 and 6, brain $5-$ HT synthesis and activity was enhanced by increasing the plasma TRP:LNAA ratio through a hydrolyzed TRP-rich protein. To induce acute stress, the Markus-Peters computerized mental arithmetic task (MPA) was used in part of the experiments (Chapters 2, 3, and 4) and the Trier Social Stress Test (TSST) was used in Chapter 6. Both stress procedures have been frequently demonstrated to induce psychological and physiological stress (e.g. Kirschbaum et al., 1993; Markus et al., 2000b; Markus et al., 2002).

In Chapter 2, healthy individuals with a family history of depression $(\mathrm{FH}+)$ and healthy controls $(\mathrm{FH}-)$ were tested in a double-blind placebo-controlled design for affective processing under acute stress exposure following ATD or placebo. Acute stress reduced responsiveness to happy words, which has been previously demonstrated for depressed patients (Murphy et al., 1999; Deveney \& Deldin, 2004; Erickson et al., 2005), supporting the hypothesis of stress as an important predecessor in the development of depression (Brown et al., 1987; Heim \& Nemeroff, 2001; Van Praag, 2004). Data from this study further revealed that the negative effects of stress on (reducing) positive affective bias may depend on family history of depression and may be influenced by ATD and task-shifting. It was concluded that $\mathrm{FH}+$ individuals may be more susceptible than $\mathrm{FH}$ - individuals to the negative effects of stress as well as to the negative effects of ATD.

In Chapter 3, we investigated the effects of ATD in subjects with different genetic 5-HTTLPR variants before and after stress exposure on affective processing. Subjects were genotyped for s allele and I allele 5HTTLPR variants and monitored for affective processes before and after stress exposure either following ATD or placebo (PLC). Data revealed 
fewer mistakes (omissions) during affective processing (on the affective go/no-go shift blocks) post-stress compared to pre-stress only in $1 / 1$ genotype. Even though 5-HTTLPR genotype did not mediate the effects of ATD, affective processing in I allele homozygotes was less affected by acute stress relative to s allele homozygotes.

In Chapter 4, we investigated the effects of ATD in subjects with different genetic 5-HTTLPR profiles before and after stress exposure on affective and cognitive-attentional changes in a double-blind placebocontrolled crossover design. Contrary to the study described in Chapter 3 , the current study accounted for the recently identified functional variants within the I allele of the 5-HTTLPR that may have underestimated the effects of 5-HTTLPR in previous studies (Hu et al., 2006; Zalsman et al., 2006). ATD lowered mood in all subjects independent of genotype. Only in $\mathrm{S}^{\prime} / \mathrm{S}^{\prime}$ genotypes, mild acute stress increased depressive mood, whereas in $L^{\prime} / L^{\prime}$ genotypes, it improved feelings of vigor. Furthermore, ATD impaired immediate recall of positive words only in $S^{\prime} / S^{\prime}$ genotypes and $S^{\prime} / S^{\prime}$ also differed from $L^{\prime} / L^{\prime}$ genotypes on measures of attention independent of treatment. We concluded that 5-HTTLPR differentially mediates responses to mild stress and ATD, suggesting greater 5-HT vulnerability of $\mathrm{S}^{\prime} / \mathrm{S}^{\prime}$ carriers to serotonergic manipulations and supporting increased depression vulnerability.

In Chapter 5, we explored whether a TRP-rich hydrolyzed protein has greater effects on the plasma TRP:LNAA ratio and mood in healthy volunteers and, thus, might be a better method to increase brain 5-HT in future studies than pure TRP and previously used intact TRP-rich alphalactalbumin whey protein. In a double-blind randomized cross-over study, plasma amino acids and mood were repeatedly measured before and after intake of intact alpha-lactalbumin and a TRP-rich hydrolyzed protein as well as after placebo protein, pure TRP, and a TRP-containing synthetic peptide. We found much greater $(>250 \%)$ increases in plasma TRP:LNAA after the TRP-rich hydrolyzed protein than after intact alphalactalbumin (67\%) and pure TRP (190\%), and mood only improved following intake of the TRP-rich hydrolyzed protein and pure TRP. Based on this data, it was concluded that a TRP-rich hydrolyzed protein may be the most adequate method to increase brain TRP and 5-HT function in future studies.

In Chapter 6, we investigated the beneficial effects of a TRP-rich hydrolyzed protein on mood and stress coping in subjects with high and 
low cognitive reactivity (CR) to depression. $C R$ is the ease with which dysfunctional schemas are activated during sad mood, which is a trait marker for depression vulnerability. In a placebo-controlled double-blind crossover study, participants' mood and cortisol was assessed before and after acute stress exposure either following intake of a TRP-rich hydrolyzed protein or placebo. The TRP-rich hydrolyzed protein significantly increased positive mood in all subjects and dampened the cortisol response to the acute stressor. No differences were found between high and low CR subjects. We concluded that a TRP-rich hydrolyzed protein has beneficial effects on mood in healthy non-vulnerable subjects and improves stress coping (likely) by improving $5-\mathrm{HT}$-related stress resilience.

Based on the results of the studies reported in the different chapters, it was concluded (Chapter 7) that $\mathrm{FH}+$ individuals and individuals with the 5-HTTLPR s allele are, indeed, more vulnerable to serotonergic manipulations than healthy non-vulnerable control participants. In addition, these serotonergic vulnerable subjects also exhibited enhanced susceptibility to the negative affective effects of acute stress exposure, supporting the assumed interaction between serotonergic vulnerability, stress, and depression. In further support of this theory, dietary-induced increases in brain 5-HT enhanced stress coping and mood in healthy subjects. Nevertheless, these interaction effects appeared to be small, which may be due to the mild nature of the stressors and should, therefore, be interpreted with caution, particularly regarding clinical relevance. Future research projects may include a more severe and sustained stress task or may focus on the development of experimental designs investigating the interaction between (early) stressful life events, serotonergic vulnerability, and depression. 

SAMENVATTING 
Affectieve stoornissen zoals depressie behoren wereldwijd tot één van de meest voorkomende ernstige ziektes welke gepaard gaan met aanzienlijke medische consequenties en toenemende mortaliteit. Het vinden van risicofactoren voor het ontstaan van depressie wordt tegenwoordig dan ook gezien als één van de meest belangrijke uitdagingen binnen de psychologie en de biologische psychiatrie. Een van de veronderstelde potentiële risicofactoren voor depressie is een genetische kwetsbaarheid of predispositie. Hoewel de exacte neurobiologische basis van een dergelijke genetische predispositie nog onvoldoende duidelijk is, blijkt de serotonine (5-HT) neurotransmitter activiteit in de hersenen een belangrijke rol te spelen. Op grond van tal van onderzoeksbevindingen werd recentelijk een zogenaamde 'serotonerge gevoeligheid' voor depressie verondersteld. Hiermee duidt men op een toegenomen kwetsbaarheid binnen het serotonerge systeem dat het risico op affectieve stoornissen doet toenemen; in het bijzonder aandoeningen als depressie die in hoge mate gerelateerd zijn aan een verstoorde serotonerge werking (Jans et al., 2007). Dit zien we bijvoorbeeld bij mensen met een familiegeschiedenis van depressie (Benkelfat et al., 1994; Klaassen et al., 1999) of bij mensen met specifieke genotypische variaties binnen het serotonine transporter gen (5-HTTLPR polymorfisme) (o.a. Neumeister et al., 2002). Het grote probleem is echter dat de rol van $5-\mathrm{HT}$ in depressie verre van eenduidig is en dat een depressieve aandoening niet direct kan worden toegeschreven aan louter een (aangeboren) serotonerge gevoeligheid. Nieuw recent onderzoek doet echter vermoeden dat een serotonerge kwetsbaarheid niet zozeer direct maar voornamelijk onder stress de kans op een depressie aanzienlijk zal vergroten. Deze interessante veronderstelling past goed bij algemeen geaccepteerde bevindingen dat stressvolle gebeurtenissen veelal vooraf gaan aan de ontwikkeling van een depressie en dat dit wordt toegeschreven aan verstoorde interacties tussen het neuroendocrine stress system en het serotonerge system. Hoewel een dergelijke interactie tussen stress, serotonerge gevoeligheid en depressie wordt ondersteund door verschillende epidemiologische studies, werd deze interactie nog niet experimenteel onderzocht. Doelstelling van dit proefschrift is dan ook om de veronderstelde interactie tussen stress en serotonerge gevoeligheid als risicofactor voor depressie experimenteel te onderzoeken en te toetsen binnen een reeks van humane studies. 
Om de interactie tussen stress, serotonerge gevoeligheid en depressie te onderzoeken werden gezonde proefpersonen met een veronderstelde depressieve en serotonerge gevoeligheid vanuit verschillende kwetsbare populaties (eerstegraads familieleden van depressieve patiënten, 5-HTTLPR s-allel dragers, en individuen met een verhoogde depressieve reactiviteit) getest na serotonine verhogende (TRPsuppletie) en verlagende (TRP depletie) manipulaties.

In de studies beschreven in hoofdstukken 2, 3, en 4 werd de 5-HT synthese en activiteit verlaagd aan de hand van de acute tryptofaan depletie (ATD) methode waarbij proefpersonen een aminozuur drankje nuttigen bestaande uit alle essentiële aminozuren met uitzondering van tryptofaan (TRP: precursor van serotonine). Inname van dit drankje veroorzaakt een drastische afname van de hoeveelheid beschikbare plasma TRP ten opzichte van de andere concurrerende aminozuren of LNAAs (TRP:LNAA ratio) voor opname in de hersenen waardoor een drastische serotonine depletie plaatsvindt (o.a. Gessa et al., 1974; Moja et al., 1991; Maes \& Meltzer, 1995). In de studies beschreven in hoofdstukken 5 en 6, werd de 5-HT synthese juist gestimuleerd door de hoeveelheid beschikbare plasma TRP voor opname in de hersenen te verhogen via consumptie van gehydrolyseerde TRP-rijke eiwitten. Om acute stress te induceren werd zowel de Markus-Peters computergestuurde mentale rekentaak (MPA: hoofdstuk 2, 3, en 4) als de Trier Social Stress Test (TSST: hoofdstuk 6) gebruikt. Beide stress procedures worden standaard gebruikt voor de inductie van psychologische en fysiologische stress (o.a. Kirschbaum et al., 1993; Markus et al., 2000b; Markus et al., 2002).

In hoofdstuk 2 werden gezonde proefpersonen met $(\mathrm{FH}+)$ of zonder (FH-) een familiare (serotonerge) gevoeligheid voor depressie getest op affectieve veranderingen onder acute stress na ATD of placebo. Resultaten laten zien dat acute stress de responsiviteit op positieve woorden vertraagd; hetgeen ook eerder is waargenomen bij depressieve mensen (Murphy et al., 1999; Deveney \& Deldin, 2004; Erickson et al., 2005). Deze bevindingen ondersteunen de hypothese dat stress een belangrijke voorloper is bij het ontstaan van depressie (Brown et al., 1987; Heim \& Nemeroff, 2001; Van Praag, 2004). Onderzoeksresultaten laten vervolgens zien dat deze negatieve effecten van stress op positieve woordherkenning (affectieve bias) afhankelijk is van familiegeschiedenis van depressie en tevens beïnvloedt wordt door ATD. Uitgaande van de aanname dat personen met een familiare geschiedenis van depressie $(\mathrm{FH}+)$ 
serotonerg gevoeliger zijn dan controle proefpersonen (FH-), wordt op grond van deze uitkomsten verondersteld dat $\mathrm{FH}+$ individuen gevoeliger zijn voor de negatieve effecten van stress en serotonine depletie vanwege een verlaagde of aflatende serotonerge functionaliteit.

In hoofdstuk 3 zijn de affectieve effecten van ATD en stress getest bij proefpersonen met en zonder een veronderstelde genetischveroorzaakte serotonerge gevoeligheid. Proefpersonen werden gescreend en genotypisch gerangschikt op de aanwezigheid van het korte (s) of lange (I) allel binnen het zogenaamde '5-HT transporter linked polymorphic region' (5-HTTLPR). Vervolgens werden bij de serotonerggevoelige (s-allel 5-HTTLPR) en controle (l-allel) proefpersonen depressie-gerelateerde affectieve processen gemeten voor en na blootstelling aan acute stress na ATD of placebo (PLC). Bevindingen laten zien dat mensen met het l-allel (controle proefpersonen) minder fouten maken tijdens affectieve verwerking onder acute stress (op de affectieve go/no-go shift blocks) dan mensen met het serotonerg gevoelige s-allel. Hoewel er geen interactie tussen 5-HTTLPR en ATD werd gevonden, blijken affectieve processen in l-allel genotypen dus minder negatief beïnvloed te worden door acute stress dan in s-allel genotypen. Met andere lijkt de afwezigheid van genetische serotonerge gevoeligheid (in Iallel dragers) juist bescherming te bieden tegen de negatieve affectieve effecten van stress.

In hoofdstuk 4 werden eveneens de effecten van ATD en stress getest op verschillende depressie-gerelateerde cognitieve en affectieve veranderingen bij mensen met verschillende genetisch gevoelige 5HTTLPR profielen. In tegenstelling tot de vorige studie, werd hierbij rekening gehouden met een recentelijk geïdentificeerde functionele variant binnen het l-allel 5-HTTLPR (de zogenaamde Lg variant) dat overeenkomt met het s-allel (en waardoor eerdere bevindingen van 5HTTLPR onderschat zouden kunnen zijn; Hu et al., 2006; Zalsman et al., 2006). ATD verlaagde de stemming bij alle proefpersonen, onafhankelijk van genotype. Echter, acute stress veroorzaakte alleen in s-allel $\left(S^{\prime} / S^{\prime}\right)$ genotypen een depressieve stemming, terwijl stress in l-allel ( $\left.L^{\prime} / L^{\prime}\right)$ genotypen juist de positieve stemming verhoogde. Bovendien verslechterde ATD de directe herinnering (immediate recall) van positieve woorden alleen in s-allel $\left(S^{\prime} / S^{\prime}\right)$ genotypen maar niet in I-allel $\left(L^{\prime} / L^{\prime}\right)$ genotypen en vertoonde de s-allel genotypen afwijkende aandachtsprocessen ten opzichte van I-allel genotypen. Deze bevindingen ondersteunen wederom 
de aanname dat s-allel 5-HTTLPR genotypen gevoeliger zijn voor de negatieve affectieve effecten van stress en ATD en dus tevens de aanname dat 5-HTTLPR de serotonerge gevoeligheid beïnvloed.

In hoofdstuk 5 werd gekeken of een TRP-rijk proteïne hydrolysaat grotere effecten heeft op de beschikbare tryptofaan voor opname in de hersenen (grotere stijging van de plasma TRP:LNAA ratio en dus 5-HT) en de stemming in gezonde vrijwilligers dan intacte alpha-lactalbumine proteïne. Het belang van deze studie was om te komen tot een betere suppletie methode ter verhoging van serotonine in de hersenen zodat deze methode vervolgens in volgende studies kan worden toegepast. In deze studie werd de beschikbare plasma TRP (in vergelijking met de andere aminozuren; TRP/LNAA ratio) en stemming herhaaldelijk gemeten voor en na inname van intacte TRP-rijke alpha-lactalbumine proteïne, gehydrolyseerde TRP-rijke proteïne, pure TRP, een synthetische TRP-rijk peptide en een placebo proteïne. Resultaten laten een aanzienlijke hogere stijging in de plasma TRP:LNAA ratio zien na inname van het gehydrolyseerde TRP-rijke proteïne in vergelijking met intacte TRP-rijke alpha-lactalbumine proteïne en zelf pure TRP. Verder werd een verbetering van de stemming geconstateerd alleen na inname van het gehydrolyseerde TRP-rijke proteïne en pure TRP. Gebaseerd op deze bevindingen werd geconcludeerd dat een gehydrolyseerde TRP-rijke proteïne de meest adequate methode is om de TRP:LNAA ratio, en dus de TRP en 5$\mathrm{HT}$ in de hersenen, te verhogen.

In hoofdstuk 6 werden de positieve effecten van een TRP-rijk proteïne hydrolysaat op stemming en stress coping getest bij proefpersonen met een hoge of lage cognitieve reactiviteit (CR) voor depressie. Cognitieve reactiviteit is een gebruikelijke vertaling van het gemak waarmee negatieve zogenaamde 'dysfunctionele' gedachten of cognitieve depressogene schema's worden geactiveerd tijdens onplezierige stemmingsverlagende momenten; hetgeen kenmerkend is voor depressiegevoeligheid. Als gevolg hiervan, in aanvulling op proefpersonen met een familiare depressieve geschiedenis of met een s-allel 5-HTTLPR zoals gehanteerd in de vorige studies, worden proefpersonen met een hoge CR ook verondersteld serotonerg gevoelig te zijn. In deze studie werd eveneens de stemming en de stress responsiviteit gemeten voor en na acute stress; echter nu na 5-HT suppletie middels inname van een TRP-rijk proteïne hydrolysaat (in vergelijking met een placebo). In tegenstelling tot placebo, verbeterde de inname van het TRP-rijk proteïne hy- 
drolysaat zowel de stemming als de stress coping (lagere stress cortisol response) in alle proefpersonen ongeacht de CR. Deze gunstige effecten van het TRP-rijk proteïne hydrolysaat op de stresscoping werden toegeschreven aan een verbeterde 5-HT functionaliteit. De afwezigheid van een effect van $C R$ werd toegeschreven aan de mogelijkheid dat $C R$ in mindere mate dan bijvoorbeeld 5-HTTLPR een serotonerge gevoeligheid typeert.

Op grond van de resultaten van onderzoeken die in de verschillende hoofdstukken zijn gerapporteerd, werd geconcludeerd (hoofdstuk 7) dat gezonde mensen met een familiaire geschiedenis van depressie $(\mathrm{FH}+)$ of met een s-allel 5-HTTLPR gevoeliger reageren op serotonerge manipulaties dan controle proefpersonen. Ook blijken zij gevoeliger of vatbaarder te zijn voor de negatieve effecten van acute stress. Deze bevinding ondersteunen niet alleen de aanname dat familiaire geschiedenis van depressie en 5-HTTLPR serotonerge gevoeligheid stimuleert voor stress en depressie, maar juist ook dat een dergelijke gevoeligheid met name onder stress de kans op depressie zou kunnen vergroten. Verder blijkt het aannemelijk dat dieetgeïnduceerde verhogingen in 5-HT stress coping en stemming verbeteren en dat het mogelijk zou kunnen zijn dat dit nog gunstiger werkt bij mensen met een familiaire en/of genetisch serotonine gevoeligheid (dit is in het huidige proefschrift niet onderzocht). $\mathrm{De}$ in dit proefschrift gevonden interactie effecten tussen serotonine gevoeligheid, stress en depressie zijn niettemin klein, wat veroorzaakt zou kunnen zijn door de relatief milde aard van de gehanteerde stressoren en zou daarom voorzichtig geïnterpreteerd moeten worden (met name omtrent klinische relevantie). Als vervolg op de huidige studies, zou toekomstig onderzoek daarom dan ook een meer krachtige of realistische stressinductie taak moeten includeren en zich tevens moeten gaan richten op de interactie tussen (vroege) chronische stressvolle levensgebeurtenissen, serotonerge gevoeligheid en depressie. 
REFERENCES 


\section{REFERENCES}

Adell, A., Garcia-Marquez, C., Armario, A., Gelpi, E., (1988). Chronic stress increases serotonin and noradrenaline in rat brain and sensitizes their responses to a further acute stress. Journal of Neurochemistry, 50, 1678-1681.

Aghajanian, G. K., Sanders-Bush, E. (2002). Serotonin. In: K. L. Davis, D. Charney, J. T. Coyle, C. Nemeroff (eds). Neuropsychopharmacology: The Fifth Generation of Progress (pp. 15-34). American College of Neuropsychopharmacology.

Akil, H. A., Morano, M. I. (1995). Stress. In: F. E. Bloom, D. J. Kupfer (eds). Psychopharmacology: The fourth generation of progress (pp. 773-785). New York: Raven Press.

Akiskal, H. S. (2005). Mood disorders: historical introduction and conceptual overview. In: B. J. Sadock, V. A. Sadock (eds). Kaplan \& Sadock's Comprehensive Textbook of Psychiatry (pp. 1559-1574). Philadelphia: Lippincott Williams \& Wilkins.

American Psychiatric Association. (1994). Diagnostic and Statistical Manual (4th ed). Washington, D. C.: American Psychiatric Association.

Arborelius, L., Owens, M. J., Plotsky, P. M., Nemeroff, C. B., (1999). The role of corticotropin-releasing factor in depression and anxiety disorders. Journal of Endocrinology, 160, $1-12$.

Arrindell, W. A., Ettema, J. H. M., (1986). SCL-90. Een multidimensionel psychopathologie indicator [SCL-90. A multidimensional indicator of psychopathology]. Lisse: Swets en Zeitlinger.

Audenaert, K., Van Laere, K., Dumont, F., Slegers, G., Mertens, J., van Heeringen, C., et al., (2001). Decreased frontal serotonin 5-HT 2a receptor binding index in deliberate self-harm patients. European Journal of Nuclear Medicine and Molecular Imaging, 28, 175-182.

Auerbach, J., Geller, V., Lezer, S., Shinwell, E., Belmaker, R. H., Levine, J., et al., (1999). Dopamine D4 receptor (D4DR) and serotonin transporter promoter (5-HTTLPR) polymorphisms in the determination of temperament in 2-month-old infants. Molecular Psychiatry, 4, 369-373.

Beck, A. T., Ward, C. H., Mendelson, M., Mock, J., Erbaugh, J., (1961). An inventory for measuring depression. Archives of General Psychiatry, 4, 561-571.

Bell, C., Abrams, J., Nutt, D., (2001). Tryptophan depletion and its implications for psychiatry. The British Journal of Psychiatry, 178, 399-405.

Benkelfat, C., Ellenbogen, M. A., Dean, P., Palmour, R. M., Young, S. N., (1994). Moodlowering effect of tryptophan depletion. Enhanced susceptibility in young men at genetic risk for major affective disorders. Archives of General Psychiatry, 51, 687-697.

Bhatti, T., Gillin, J. C., Seifritz, E., Moore, P., Clark, C., Golshan, S., et al., (1998). Effects of a tryptophan-free amino acid drink challenge on normal human sleep electroencephalogram and mood. Biological Psychiatry, 43, 52-59.

Biegon, A., Grinspoon, A., Blumenfeld, B., Bleich, A., Apter, A., Mester, R., (1990). Increased serotonin $5-\mathrm{HT} 2$ receptor binding on blood platelets of suicidal men. Psychopharmacology (Berl), 100, 165-167.

Biggio, G., Fadda, F., Fanni, P., Tagliamonte, A., Gessa, G. L., (1974). Rapid depletion of serum tryptophan, brain tryptophan, serotonin and 5-hydroxyindoleacetic acid by a tryptophan-free diet. Life Sciences, 14, 1321-1329.

Blokland, A., Lieben, C., Deutz, N. E. P., Schmitt, J., (2004). Acute tryptophan depletion: comparing the effects of an amino acid mixture with a gelatin-based protein in man and rats. Current Topics in Nutraceutical Research, 2, 161-169. 
Bondy, B., Erfurth, A., de Jonge, S., Kruger, M., Meyer, H., (2000). Possible association of the short allele of the serotonin transporter promoter gene polymorphism (5-HTTLPR) with violent suicide. Molecular Psychiatry, 5, 193-195.

Booij, L., Merens, W., Markus, C. R., Van der Does, A. J., (2006). Diet rich in \{alpha\}lactalbumin improves memory in unmedicated recovered depressed patients and matched controls. Journal of Psychopharmacology, 20, 526-535.

Booij, L., Van der Does, A. J., (2007). Cognitive and serotonergic vulnerability to depression: convergent findings. Journal of Abnormal Psychology, 116, 86-94.

Booij, L., van der Does, A. J., Haffmans, P. M., Spinhoven, P., McNally, R. J., (2005). Acute tryptophan depletion as a model of depressive relapse: behavioural specificity and ethical considerations. The British Journal of Psychiatry, 187, 148-154.

Bradley, B. P., Mogg, K., Williams, R., (1995). Implicit and explicit memory for emotioncongruent information in clinical depression and anxiety. Behaviour Research and Therapy, 33, 755-770.

Brown, G. W., Bifulco, A., Harris, T. O., (1987). Life events, vulnerability and onset of depression: some refinements. The British Journal of Psychiatry, 150, 30-42.

Cannon, W. B., (1932). The wisdom of the body. New York: Norton.

Carpenter, L. L., Anderson, G. M., Pelton, G. H., Gudin, J. A., Kirwin, P. D., Price, L. H., et al., (1998). Tryptophan depletion during continuous CSF sampling in healthy human subjects. Neuropsychopharmacology, 19, 26-35.

Carroll, B. J., (1982). Use of the dexamethasone suppression test in depression. Journal of Clinical Psychiatry, 43, 44-50.

Caspi, A., Sugden, K., Moffitt, T. E., Taylor, A., Craig, I. W., Harrington, H., et al., (2003). Influence of life stress on depression: moderation by a polymorphism in the 5-HTT gene. Science, 301, 386-389.

Clow, A. (2001). The physiology of stress. In: F. Jones, J. Bright (eds). Stress: myth, theory and research (pp. 47-61). London: Pearson Education Limited.

Collier, D. A., Stober, G., Li, T., Heils, A., Catalano, M., Di Bella, D., et al., (1996). A novel functional polymorphism within the promoter of the serotonin transporter gene: possible role in susceptibility to affective disorders. Molecular Psychiatry, 1, 453-460.

Curzon, G. (1985). Effects of food intake on brain transmitter amine precursors and amine synthesis. In: M. Sandler, T. Silverstone (eds). Psychopharmacology and food (pp. 5970). Oxford: Oxford University Press.

Davis, S., Heal, D. J., Stanford, S. C., (1995). Long-lasting effects of an acute stress on the neurochemistry and function of 5-hydroxytryptaminergic neurones in the mouse brain. Psychopharmacology (Berl), 118, 267-272.

Dayan, P., Huys, Q. J., (2008). Serotonin, inhibition, and negative mood. PLoS Computational Biology, 4, e4.

De Kloet, E. R., Joels, M., Holsboer, F., (2005). Stress and the brain: from adaptation to disease. Nature Review Neuroscience, 6, 463-475.

De Kloet, E. R., Kovacs, G. L., Szabo, G., Telegdy, G., Bohus, B., Versteeg, D. H., (1982). Decreased serotonin turnover in the dorsal hippocampus of rat brain shortly after adrenalectomy: selective normalization after corticosterone substitution. Brain Research, 239, 659-663.

De Kloet, E. R., Versteeg, D. H., Kovacs, G. L., (1983). Aldosterone blocks the response to corticosterone in the raphe-hippocampal serotonin system. Brain Research, 264, 323327. 


\section{REFERENCES}

Deakin, J. F., Graeff, F. G., (1991). 5-HT and mechanisms of defence. Journal of Psychopharmacology, 5

Delgado, P. L., Charney, D. S., Price, L. H., Aghajanian, G. K., Landis, H., Heninger, G. R., (1990). Serotonin function and the mechanism of antidepressant action. Reversal of antidepressant-induced remission by rapid depletion of plasma tryptophan. Archives of General Psychiatry, 47, 411-418.

Delgado, P. L., Miller, H. L., Salomon, R. M., Licinio, J., Krystal, J. H., Moreno, F. A., et al., (1999). Tryptophan-depletion challenge in depressed patients treated with desipramine or fluoxetine: implications for the role of serotonin in the mechanism of antidepressant action. Biological Psychiatry, 46, 212-220.

Delgado, P. L., Price, L. H., Miller, H. L., Salomon, R. M., Aghajanian, G. K., Heninger, G. R., et al., (1994). Serotonin and the neurobiology of depression. Effects of tryptophan depletion in drug-free depressed patients. Archives of General Psychiatry, 51, 865-874.

Deveney, C. M., Deldin, P. J., (2004). Memory of faces: a slow wave ERP study of major depression. Emotion, 4, 295-304.

Dickerson, S. S., Kemeny, M. E., (2004). Acute stressors and cortisol responses: a theoretical integration and synthesis of laboratory research. Psychological Bulletin, 130, 355391.

Dinan, T. G., (1994). Glucocorticoids and the genesis of depressive illness. A psychobiological model. The British Journal of Psychiatry, 164, 365-371.

Dinan, T. G., (1996). Serotonin and the regulation of hypothalamic-pituitary-adrenal axis function. Life Sciences, 58, 1683-1694.

Domino, E. F. (1976). Pharmacokinetics of oral tryptophan in drug-free psychistric patients. In: L. A. Gottschalk, E. Merkis (eds). Pharmacokinetics of psychoactive drugs (pp. 117126). New York: Spektrum Publications.

Drevets, W. C., Frank, E., Price, J. C., Kupfer, D. J., Holt, D., Greer, P. J., et al., (1999). PET imaging of serotonin $1 \mathrm{~A}$ receptor binding in depression. Biological Psychiatry, 46, 1375-1387.

Eley, T. C., Sugden, K., Corsico, A., Gregory, A. M., Sham, P., McGuffin, P., et al., (2004). Gene-environment interaction analysis of serotonin system markers with adolescent depression. Molecular Psychiatry, 9, 908-915.

Elliott, R., Sahakian, B. J., McKay, A. P., Herrod, J. J., Robbins, T. W., Paykel, E. S., (1996). Neuropsychological impairments in unipolar depression: the influence of perceived failure on subsequent performance. Psychological Medicine, 26, 975-989.

Erickson, K., Drevets, W. C., Clark, L., Cannon, D. M., Bain, E. E., Zarate, C. A., Jr., et al., (2005). Mood-congruent bias in affective go/no-go performance of unmedicated patients with major depressive disorder. The American Journal of Psychiatry, 162, 21712173.

Evers, E. A., Tillie, D. E., van der Veen, F. M., Lieben, C. K., Jolles, J., Deutz, N. E., et al., (2005). Effects of a novel method of acute tryptophan depletion on plasma tryptophan and cognitive performance in healthy volunteers. Psychopharmacology (Berl), 178, 9299.

Fernstrom, J. D., (1990). Aromatic amino acids and monoamine synthesis in the central nervous system: influence of the diet. The Journal of Nutritional Biochemistry, 1, 508517.

Fernstrom, J. D., Larin, F., Wurtman, R. J., (1973). Correlations between brain tryptophan and plasma neutral amino acids levels following food consumption in rats. Life Sciences, 13, 517. 
Fernstrom, J. D., Wurtman, R. J., (1971). Brain serotonin content: physiological dependence on plasma tryptophan levels. Science, 173, 149-152.

Fernstrom, J. D., Wurtman, R. J., (1972). Brain serotonin content: physiological regulation by plasma neutral amino acids. Science, 178, 414-416.

Firk, C., Markus, C. R., (2007). Review: Serotonin by stress interaction: a susceptibility factor for the development of depression? Journal of Psychopharmacology, 21, 538-544.

Firk, C., Markus, C. R., (2008). Effects of acute tryptophan depletion on affective processing in first-degree relatives of depressive patients and controls after exposure to uncontrollable stress. Psychopharmacology (Berl), 199, 151-160.

Fuchs, E., Flugge, G., (2003). Chronic social stress: effects on limbic brain structures. Physiology \& Behavior, 79, 417-427.

Fulford, A. J., Harbuz, M. S. (2005). An introduction to the HPA axis. In: T. Steckler, N. H. Kalin, J. M. H. M. Reul (eds). Handbook of Stress and the Brain (pp. 43-65). Amsterdam: Elsevier B. V.

Fuller, R. W., (1996). Serotonin receptors involved in regulation of pituitary-adrenocortical function in rats. Behavioural Brain Research, 73, 215-219.

Fusar-Poli, P., Allen, P., Lee, F., Surguladze, S., Tunstall, N., Fu, C. H., et al., (2007). Modulation of neural response to happy and sad faces by acute tryptophan depletion. Psychopharmacology (Berl)

Fusar-Poli, P., Allen, P., McGuire, P., Placentino, A., Cortesi, M., Perez, J., (2006). Neuroimaging and electrophysiological studies of the effects of acute tryptophan depletion: a systematic review of the literature. Psychopharmacology (Berl), 188, 131-143.

Gessa, G. L., Biggio, G., Fadda, F., Corsini, G. U., Tagliamonte, A., (1974). Effect of the oral administration of tryptophan-free amino acid mixtures on serum tryptophan, brain tryptophan and serotonin metabolism. Journal of Neurochemistry, 22, 869-870.

Gillespie, N. A., Whitfield, J. B., Williams, B., Heath, A. C., Martin, N. G., (2005). The relationship between stressful life events, the serotonin transporter (5-HTTLPR) genotype and major depression. Psychological Medicine, 35, 101-111.

Glatz, K., Mossner, R., Heils, A., Lesch, K. P., (2003). Glucocorticoid-regulated human serotonin transporter (5-HTT) expression is modulated by the 5-HTT gene-promotor-linked polymorphic region. Journal of Neurochemistry, 86, 1072-1078.

Gonda, X., Juhasz, G., Laszik, A., Rihmer, Z., Bagdy, G., (2005). Subthreshold depression is linked to the functional polymorphism of the $5 \mathrm{HT}$ transporter gene. Journal of Affective Disorders, 87, 291-297.

Gonda, X., Rihmer, Z., Zsombok, T., Bagdy, G., Akiskal, K. K., Akiskal, H. S., (2006). The 5 HTTLPR polymorphism of the serotonin transporter gene is associated with affective temperaments as measured by TEMPS-A. Journal of Affective Disorders, 91, 125-131.

Gotlib, I. H., Joormann, J., Minor, K. L., Hallmayer, J., (2008). HPA axis reactivity: a mechanism underlying the associations among 5-HTTLPR, stress, and depression. Biological Psychiatry, 63, 847-851.

Grabe, H. J., Lange, M., Wolff, B., Volzke, H., Lucht, M., Freyberger, H. J., et al., (2005). Mental and physical distress is modulated by a polymorphism in the 5-HT transporter gene interacting with social stressors and chronic disease burden. Molecular Psychiatry, 10, 220-224.

Greenberg, B. D., Tolliver, T. J., Huang, S. J., Li, Q., Bengel, D., Murphy, D. L., (1999). Genetic variation in the serotonin transporter promoter region affects serotonin uptake in human blood platelets. American Journal of Medicine Genetics, 88, 83-87. 


\section{REFERENCES}

Hariri, A. R., Drabant, E. M., Munoz, K. E., Kolachana, B. S., Mattay, V. S., Egan, M. F., et al., (2005). A susceptibility gene for affective disorders and the response of the human amygdala. Archives of General Psychiatry, 62, 146-152.

Hariri, A. R., Mattay, V. S., Tessitore, A., Kolachana, B., Fera, F., Goldman, D., et al., (2002). Serotonin transporter genetic variation and the response of the human amygdala. Science, 297, 400-403.

Hayward, G., Goodwin, G. M., Cowen, P. J., Harmer, C. J., (2005). Low-dose tryptophan depletion in recovered depressed patients induces changes in cognitive processing without depressive symptoms. Biological Psychiatry, 57, 517-524.

Heils, A., Teufel, A., Petri, S., Stober, G., Riederer, P., Bengel, D., et al., (1996). Allelic variation of human serotonin transporter gene expression. Journal of Neurochemistry, 66, 2621-2624.

Heim, C., Nemeroff, C. B., (2001). The role of childhood trauma in the neurobiology of mood and anxiety disorders: preclinical and clinical studies. Biological Psychiatry, 49, 1023-1039.

Heim, C., Newport, D. J., Mletzko, T., Miller, A. H., Nemeroff, C. B., (2008). The link between childhood trauma and depression: insights from HPA axis studies in humans. Psychoneuroendocrinology, 33, 693-710.

Heine, W., Radke, M., Wutzke, K. D., Peters, E., Kundt, G., (1996). alpha-Lactalbuminenriched low-protein infant formulas: a comparison to breast milk feeding. Acta Paediatrica, 85, 1024-1028.

Heinz, A., Braus, D. F., Smolka, M. N., Wrase, J., Puls, I., Hermann, D., et al., (2005). Amygdala-prefrontal coupling depends on a genetic variation of the serotonin transporter. Nature Neuroscience, 8, 20-21.

Hermans, D., De Houwer, J., (1994). Affective and subjective familiarity ratings of 740 dutch words. Psychologica Belgica, 34, 115-139.

Holsboer, F., (2000). The corticosteroid receptor hypothesis of depression. Neuropsychopharmacology, 23, 477-501.

Holsboer, F., Gerken, A., von Bardeleben, U., Grimm, W., Beyer, H., Muller, O. A., et al., (1986). Human corticotropin-releasing hormone in depression--correlation with thyrotropin secretion following thyrotropin-releasing hormone. Biological Psychiatry, 21, 601-611.

Holsboer, F., Lauer, C. J., Schreiber, W., Krieg, J. C., (1995). Altered hypothalamicpituitary-adrenocortical regulation in healthy subjects at high familial risk for affective disorders. Neuroendocrinology, 62, 340-347.

Hu, X., Oroszi, G., Chun, J., Smith, T. L., Goldman, D., Schuckit, M. A., (2005). An expanded evaluation of the relationship of four alleles to the level of response to alcohol and the alcoholism risk. Alcoholism: Clinical and Experimental Research, 29, 8-16.

Hu, X. Z., Lipsky, R. H., Zhu, G., Akhtar, L. A., Taubman, J., Greenberg, B. D., et al., (2006). Serotonin transporter promoter gain-of-function genotypes are linked to obsessivecompulsive disorder. The American Journal of Human Genetics, 78, 815-826.

Jacobs, N., Kenis, G., Peeters, F., Derom, C., Vlietinck, R., van Os, J., (2006). Stress-related negative affectivity and genetically altered serotonin transporter function: evidence of synergism in shaping risk of depression. Archives of General Psychiatry, 63, 989-996.

Jans, L. A., Riedel, W. J., Markus, C. R., Blokland, A., (2007). Serotonergic vulnerability and depression: assumptions, experimental evidence and implications. Molecular Psychiatry, 12, 522-543. 
Joiner, T. E., Jr., Johnson, F., Soderstrom, K., Brown, J. S., (2003). Is there an association between serotonin transporter gene polymorphism and family history of depression? Journal of Affective Disorders, 77, 273-275.

Kaufman, J., Yang, B. Z., Douglas-Palumberi, H., Houshyar, S., Lipschitz, D., Krystal, J. H., et al., (2004). Social supports and serotonin transporter gene moderate depression in maltreated children. The Proceedings of the National Academy of Sciences, 101, 17316-17321.

Kelsoe, J. R. (2005). Mood disoders: genetics. In: B. J. Sadock, V. A. Sadock (eds). Kaplan \& Sadock's Comprehensive Textbook of Psychiatry (pp. 1582-1593). Philadelphia: Lippincott Williams \& Wilkins.

Kendler, K. S., Gardner, C. O., (2001). Monozygotic twins discordant for major depression: a preliminary exploration of the role of environmental experiences in the aetiology and course of illness. Psychological Medicine, 31, 411-423.

Kendler, K. S., Kuhn, J. W., Vittum, J., Prescott, C. A., Riley, B., (2005). The interaction of stressful life events and a serotonin transporter polymorphism in the prediction of episodes of major depression: a replication. Archives of General Psychiatry, 62, 529-535.

Kirschbaum, C., Kudielka, B. M., Gaab, J., Schommer, N. C., Hellhammer, D. H., (1999). Impact of gender, menstrual cycle phase, and oral contraceptives on the activity of the hypothalamus-pituitary-adrenal axis. Psychosomatic Medicine, 61, 154-162.

Kirschbaum, C., Pirke, K. M., Hellhammer, D. H., (1993). The 'Trier Social Stress Test'--a tool for investigating psychobiological stress responses in a laboratory setting. Neuropsychobiology, 28, 76-81.

Kirschbaum, C., Wolf, O. T., May, M., Wippich, W., Hellhammer, D. H., (1996). Stress- and treatment-induced elevations of cortisol levels associated with impaired declarative memory in healthy adults. Life Sciences, 58, 1475-1483.

Klaassen, T., Riedel, W. J., Deutz, N. E., Van Praag, H. M., (2002). Mood congruent memory bias induced by tryptophan depletion. Psychological Medicine, 32, 167-172.

Klaassen, T., Riedel, W. J., van Someren, A., Deutz, N. E., Honig, A., van Praag, H. M., (1999). Mood effects of 24-hour tryptophan depletion in healthy first-degree relatives of patients with affective disorders. Biological Psychiatry, 46, 489-497.

Kudielka, B. M., Schommer, N. C., Hellhammer, D. H., Kirschbaum, C., (2004). Acute HPA axis responses, heart rate, and mood changes to psychosocial stress (TSST) in humans at different times of day. Psychoneuroendocrinology, 29, 983-992.

Lau, M. A., Segal, Z. V., Williams, J. M., (2004). Teasdale's differential activation hypothesis: implications for mechanisms of depressive relapse and suicidal behaviour. Behaviour Research and Therapy, 42, 1001-1017.

Lefebvre, H., Contesse, V., Delarue, C., Feuilloley, M., Hery, F., Grise, P., et al., (1992). Serotonin-induced stimulation of cortisol secretion from human adrenocortical tissue is mediated through activation of a serotonin4 receptor subtype. Neuroscience, 47, 9991007.

Lesch, K. P., Balling, U., Gross, J., Strauss, K., Wolozin, B. L., Murphy, D. L., et al., (1994). Organization of the human serotonin transporter gene. Journal of Neural Transmission General Section, 95, 157-162.

Lesch, K. P., Bengel, D., Heils, A., Sabol, S. Z., Greenberg, B. D., Petri, S., et al., (1996). Association of anxiety-related traits with a polymorphism in the serotonin transporter gene regulatory region. Science, 274, 1527-1531. 


\section{REFERENCES}

Lezak, M. D. (2004). Auditory-Verbal Learning Test (AVLT). In: M. D. Lezak, D. B. Howieson, D. W. Loring (eds). Neuropsychological assessment, 4th ed. (pp. 422-429). London: Oxford University Press.

Lieb, R., Isensee, B., Hofler, M., Wittchen, H. U., (2002). Parental depression and depression in offspring: evidence for familial characteristics and subtypes? Journal of Psychiatric Research, 36, 237-246.

Lieberman, H. R., Wurtman, J. J., Chew, B., (1986). Changes in mood after carbohydrate consumption among obese individuals. American Journal of Clinical Nutrition, 44, $772-$ 778.

Lim, S. L., Kim, J. H., (2005). Cognitive processing of emotional information in depression, panic, and somatoform disorder. Journal of Abnormal Psychology, 114, 50-61.

Lopez, J. F., Chalmers, D. T., Little, K. Y., Watson, S. J., (1998). A.E. Bennett Research Award. Regulation of serotonin1A, glucocorticoid, and mineralocorticoid receptor in rat and human hippocampus: implications for the neurobiology of depression. Biological Psychiatry, 43, 547-573.

Lotrich, F. E., Pollock, B. G., (2004). Meta-analysis of serotonin transporter polymorphisms and affective disorders. Psychiatric Genetics, 14, 121-129.

Lupien, S. J., Maheu, F., Tu, M., Fiocco, A., Schramek, T. E., (2007). The effects of stress and stress hormones on human cognition: Implications for the field of brain and cognition. Brain and Cognition, 65, 209-237.

Maes, M., Meltzer, H. Y. (1995). The serotonin hypothesis of major depression. In: F. E. Bloom, D. J. Kupfer (eds). Psychopharmacology: The Fourth Generation of Progress (pp. 933-944). New York: Raven Press.

Maes, M., Minner, B., Suy, E., (1991). The relationships between the availabilityof Ltryptophan to the brain, the spontaneous HPA-axis activity, and the HPA-axis responses to dexamethasone in depressed patients. Amino Acids, 1, 57-65.

Malison, R. T., Price, L. H., Berman, R., van Dyck, C. H., Pelton, G. H., Carpenter, L., et al., (1998). Reduced brain serotonin transporter availability in major depression as measured by [123I]-2 beta-carbomethoxy-3 beta-(4-iodophenyl)tropane and single photon emission computed tomography. Biological Psychiatry, 44, 1090-1098.

Mann, J. J., Huang, Y. Y., Underwood, M. D., Kassir, S. A., Oppenheim, S., Kelly, T. M., et al., (2000). A serotonin transporter gene promoter polymorphism (5-HTTLPR) and prefrontal cortical binding in major depression and suicide. Archives of General Psychiatry, 57, 729-738.

Markus, C. R. (2003). Food, stress and mood. In: D. Wadson, F. Dodds (eds). Performance Functional Foods.Cambridge: Woodhead Publishing Limited.

Markus, C. R., (2007). Effects of carbohydrates on brain tryptophan availability and stress performance. Biological Psychology, 76, 83-90.

Markus, C. R., (2008). Dietary Amino Acids and Brain Serotonin Function; Implications for Stress-Related Affective Changes. Neuromolecular Medicine [Epub ahead of print].

Markus, C. R., Firk, C., Gerhardt, C., Kloek, J., Smolders, G. F., (2008). Effect of different tryptophan sources on amino acids availability to the brain and mood in healthy volunteers. Psychopharmacology (Berl), 201, 107-114.

Markus, C. R., Jonkman, L. M., Lammers, J. H., Deutz, N. E., Messer, M. H., Rigtering, N., (2005). Evening intake of alpha-lactalbumin increases plasma tryptophan availability and improves morning alertness and brain measures of attention. American Journal of Clinical Nutrition, 81, 1026-1033. 
Markus, C. R., Olivier, B., de Haan, E. H., (2002). Whey protein rich in alpha-lactalbumin increases the ratio of plasma tryptophan to the sum of the other large neutral amino acids and improves cognitive performance in stress-vulnerable subjects. American Journal of Clinical Nutrition, 75, 1051-1056.

Markus, C. R., Olivier, B., Panhuysen, G. E., Van Der Gugten, J., Alles, M. S., Tuiten, A., et al., (2000a). The bovine protein alpha-lactalbumin increases the plasma ratio of tryptophan to the other large neutral amino acids, and in vulnerable subjects raises brain serotonin activity, reduces cortisol concentration, and improves mood under stress. American Journal of Clinical Nutrition, 71, 1536-1544.

Markus, C. R., Panhuysen, G., Jonkman, L. M., Bachman, M., (1999). Carbohydrate intake improves cognitive performance of stress-prone individuals under controllable laboratory stress. British Journal of Nutrition, 82, 457-467.

Markus, C. R., Panhuysen, G., Tuiten, A., Koppeschaar, H., (2000b). Effects of food on cortisol and mood in vulnerable subjects under controllable and uncontrollable stress. Physiology \& Behavior, 70, 333-342.

Markus, C. R., Panhuysen, G., Tuiten, A., Koppeschaar, H., Fekkes, D., Peters, M. L., (1998). Does carbohydrate-rich, protein-poor food prevent a deterioration of mood and cognitive performance of stress-prone subjects when subjected to a stressful task? Appetite, 31, 49-65.

Marsh, A. A., Finger, E. C., Buzas, B., Soliman, N., Richell, R. A., Vythilingham, M., et al., (2006). Impaired recognition of fear facial expressions in 5-HTTLPR S-polymorphism carriers following tryptophan depletion. Psychopharmacology (Berl), 189, 387-394.

Mason, J. W., (1968). A review of psychoendocrine research on the pituitary-adrenal cortical system. Psychosomatic Medicine, 30, Suppl:576-607.

Matt, G. E., Vazquez, C., Campbell, W. K., (1992). Mood-congruent recall of affectively toned stimuli: a meta-analytic review. Clinical Psychology Review, 12, 227-255.

McEwen, B. S., (2000). Allostasis and allostatic load: implications for neuropsychopharmacology. Neuropsychopharmacology, 22, 108-124.

Merens, W., Booij, L., Markus, R., Zitman, F. G., Onkenhout, W., Van der Does, A. J., (2005). The effects of a diet enriched with alpha-lactalbumin on mood and cortisol response in unmedicated recovered depressed subjects and controls. British Journal of Nutrition, 94, 415-422.

Meyer, J. H., Wilson, A. A., Sagrati, S., Hussey, D., Carella, A., Potter, W. Z., et al., (2004). Serotonin transporter occupancy of five selective serotonin reuptake inhibitors at different doses: an [11C]DASB positron emission tomography study. The American Journal of Psychiatry, 161, 826-835.

Minet-Ringuet, J., Le Ruyet, P. M., Tome, D., Even, P. C., (2004). A tryptophan-rich protein diet efficiently restores sleep after food deprivation in the rat. Behavioural Brain Research, 152, 335-340.

Modell, S., Lauer, C. J., Schreiber, W., Huber, J., Krieg, J. C., Holsboer, F., (1998). Hormonal response pattern in the combined DEX-CRH test is stable over time in subjects at high familial risk for affective disorders. Neuropsychopharmacology, 18, 253-262.

Modell, S., Yassouridis, A., Huber, J., Holsboer, F., (1997). Corticosteroid receptor function is decreased in depressed patients. Neuroendocrinology, 65, 216-222.

Mogg, K., Bradley, B. P., (2005). Attentional bias in generalized anxiety disorder versus depressive disorder. Cognitive Therapy and Research, 29, 29-45. 


\section{REFERENCES}

Moja, E. A., Cipolla, P., Castoldi, D., Tofanetti, O., (1989). Dose-response decrease in plasma tryptophan and in brain tryptophan and serotonin after tryptophan-free amino acid mixtures in rats. Life Sciences, 44, 971-976.

Moja, E. A., Rocchi, E., Benedetti, F., Paolillo, F., Casalgrandi, G., Ponz de Leon, M., (1991). Decrease in plasma tryptophan after a tryptophan-free amino acid solution. A comparison between cirrhotic and control subjects. Life Sciences, 48, 409-418.

Monsell, S., (2003). Task switching. Trends in Cognitive Sciences, 7, 134-140.

Moreno, F. A., Heninger, G. R., McGahuey, C. A., Delgado, P. L., (2000). Tryptophan depletion and risk of depression relapse: a prospective study of tryptophan depletion as a potential predictor of depressive episodes. Biological Psychiatry, 48, 327-329.

Moreno, F. A., McGahuey, C. A., Freeman, M. P., Delgado, P. L., (2006). Sex differences in depressive response during monoamine depletions in remitted depressive subjects. Journal of Clinical Psychiatry, 67, 1618-1623.

Murphy, F. C., Sahakian, B. J., Rubinsztein, J. S., Michael, A., Rogers, R. D., Robbins, T. W., et al., (1999). Emotional bias and inhibitory control processes in mania and depression. Psychological Medicine, 29, 1307-1321.

Murphy, F. C., Smith, K. A., Cowen, P. J., Robbins, T. W., Sahakian, B. J., (2002). The effects of tryptophan depletion on cognitive and affective processing in healthy volunteers. Psychopharmacology (Berl), 163, 42-53.

Nestler, E. J., Barrot, M., DiLeone, R. J., Eisch, A. J., Gold, S. J., Monteggia, L. M., (2002). Neurobiology of depression. Neuron, 34, 13-25.

Neumeister, A., Bain, E., Nugent, A. C., Carson, R. E., Bonne, O., Luckenbaugh, D. A., et al., (2004a). Reduced serotonin type 1A receptor binding in panic disorder. The Journal of Neuroscience, 24, 589-591.

Neumeister, A., Hu, X. Z., Luckenbaugh, D. A., Schwarz, M., Nugent, A. C., Bonne, O., et al., (2006). Differential Effects of 5-HTTLPR Genotypes on the Behavioral and Neural Responses to Tryptophan Depletion in Patients With Major Depression and Controls. Archives of General Psychiatry, 63, 978-986.

Neumeister, A., Konstantinidis, A., Stastny, J., Schwarz, M. J., Vitouch, O., Willeit, M., et al., (2002). Association between serotonin transporter gene promoter polymorphism (5HTTLPR) and behavioral responses to tryptophan depletion in healthy women with and without family history of depression. Archives of General Psychiatry, 59, 613-620.

Neumeister, A., Nugent, A. C., Waldeck, T., Geraci, M., Schwarz, M., Bonne, O., et al., (2004b). Neural and behavioral responses to tryptophan depletion in unmedicated patients with remitted major depressive disorder and controls. Archives of General Psychiatry, 61, 765-773.

Neumeister, A., Young, T., Stastny, J., (2004c). Implications of genetic research on the role of the serotonin in depression: emphasis on the serotonin type $1 \mathrm{~A}$ receptor and the serotonin transporter. Psychopharmacology (Berl), 174, 512-524.

Nishizawa, S., Benkelfat, C., Young, S. N., Leyton, M., Mzengeza, S., de Montigny, C., et al., (1997). Differences between males and females in rates of serotonin synthesis in human brain. The Proceedings of the National Academy of Sciences, 94, 5308-5313.

Nuller, J. L., Ostroumova, M. N., (1980). Resistance to inhibiting effect of dexamethasone in patients with endogenous depression. Acta Psychiatrica Scandinavica, 61, 169-177.

Orosco, M., Rouch, C., Beslot, F., Feurte, S., Regnault, A., Dauge, V., (2004). Alphalactalbumin-enriched diets enhance serotonin release and induce anxiolytic and rewarding effects in the rat. Behavioural Brain Research, 148, 1-10. 
Owens, M. J., Nemeroff, C. B., (1994). Role of serotonin in the pathophysiology of depression: focus on the serotonin transporter. Clinical Chemistry, 40, 288-295.

Paelecke-Habermann, Y., Pohl, J., Leplow, B., (2005). Attention and executive functions in remitted major depression patients. Journal of Affective Disorders, 89, 125-135.

Pandey, G. N., Pandey, S. C., Janicak, P. G., Marks, R. C., Davis, J. M., (1990). Platelet serotonin-2 receptor binding sites in depression and suicide. Biological Psychiatry, 28, 215222.

Perreau-Linck, E., Beauregard, M., Gravel, P., Paquette, V., Soucy, J. P., Diksic, M., et al., (2007). In vivo measurements of brain trapping of C-labelled alpha-methyl-Ltryptophan during acute changes in mood states. Journal of Psychiatry \& Neuroscience, 32, 430-434.

Peters, M. L., Godaert, G. L., Ballieux, R. E., van Vliet, M., Willemsen, J. J., Sweep, F. C., et al., (1998). Cardiovascular and endocrine responses to experimental stress: effects of mental effort and controllability. Psychoneuroendocrinology, 23, 1-17.

Pezawas, L., Meyer-Lindenberg, A., Drabant, E. M., Verchinski, B. A., Munoz, K. E., Kolachana, B. S., et al., (2005). 5-HTTLPR polymorphism impacts human cingulateamygdala interactions: a genetic susceptibility mechanism for depression. Nature Neuroscience, 8, 828-834.

Porter, R. J., Gallagher, P., Thompson, J. M., Young, A. H., (2003). Neurocognitive impairment in drug-free patients with major depressive disorder. The British Journal of Psychiatry, 182, 214-220.

Porter, R. J., Gallagher, P., Watson, S., Young, A. H., (2004). Corticosteroid-serotonin interactions in depression: a review of the human evidence. Psychopharmacology (Berl), $173,1-17$.

Rao, U., Hammen, C., Ortiz, L. R., Chen, L. A., Poland, R. E., (2008). Effects of early and recent adverse experiences on adrenal response to psychosocial stress in depressed adolescents. Biological Psychiatry, 64, 521-526.

Reich, T., Van Eerdewegh, P., Rice, J., Mullaney, J., Endicott, J., Klerman, G. L., (1987). The familial transmission of primary major depressive disorder. Journal of Psychiatric Research, 21, 613-624.

Richell, R. A., Deakin, J. F., Anderson, I. M., (2005). Effect of acute tryptophan depletion on the response to controllable and uncontrollable noise stress. Biological Psychiatry, 57, 295-300.

Riedel, W. J., Klaassen, T., Deutz, N. E., van Someren, A., van Praag, H. M., (1999). Tryptophan depletion in normal volunteers produces selective impairment in memory consolidation. Psychopharmacology (Berl), 141, 362-369.

Rihmer, Z., Angst, J. (2005). Mood disorders: epidemiology. In: B. J. Sadock, V. A. Sadock (eds). Kaplan \& Sadock's Comprehensice Textbook of Psychiatry (pp. 1575-1581). Philadelphia: Lippincott Williams \& Wilkins.

Rinck, M., Becker, E. S., (2005). A comparison of attentional biases and memory biases in women with social phobia and major depression. Journal of Abnormal Psychology, $114,62-74$.

Roiser, J. P., Blackwell, A. D., Cools, R., Clark, L., Rubinsztein, D. C., Robbins, T. W., et al., (2006). Serotonin Transporter Polymorphism Mediates Vulnerability to Loss of Incentive Motivation Following Acute Tryptophan Depletion. Neuropsychopharmacology, 31, 2264-2272

Roiser, J. P., Cook, L. J., Cooper, J. D., Rubinsztein, D. C., Sahakian, B. J., (2005). Association of a functional polymorphism in the serotonin transporter gene with abnormal 


\section{REFERENCES}

emotional processing in ecstasy users. The American Journal of Psychiatry, 162, 609612.

Roiser, J. P., Muller, U., Clark, L., Sahakian, B. J., (2007). The effects of acute tryptophan depletion and serotonin transporter polymorphism on emotional processing in memory and attention. International Journal of Neuropsychopharmacology, 10, 449-461.

Rosenthal, N. E., Genhart, M. J., Caballero, B., Jacobsen, F. M., Skwerer, R. G., Coursey, R. D., et al., (1989). Psychobiological effects of carbohydrate- and protein-rich meals in patients with seasonal affective disorder and normal controls. Biological Psychiatry, 25, 1029-1040.

Rubinsztein, J. S., Rogers, R. D., Riedel, W. J., Mehta, M. A., Robbins, T. W., Sahakian, B. J., (2001). Acute dietary tryptophan depletion impairs maintenance of "affective set" and delayed visual recognition in healthy volunteers. Psychopharmacology (Berl), 154, 319-326.

Ruhe, H. G., Mason, N. S., Schene, A. H., (2007). Mood is indirectly related to serotonin, norepinephrine and dopamine levels in humans: a meta-analysis of monoamine depletion studies. Molecular Psychiatry, 12, 331-359.

Rybakowski, J. K., Twardowska, K., (1999). The dexamethasone/corticotropin-releasing hormone test in depression in bipolar and unipolar affective illness. Journal of Psychiatric Research, 33, 363-370.

Sambeth, A., Riedel, W. J., Tillie, D. E., Blokland, A., Postma, A., Schmitt, J. A., (2008). Memory impairments in humans after acute tryptophan depletion using a novel gelatinbased protein drink. Journal of Psychopharmacology

Sapolsky, R. M., (1992). Stress, the Aging Brain, and the Mechanism of Neuron Death. Cambridge, MA: MIT Press.

Sapolsky, R. M., (1996). Why stress is bad for your brain. Science, 273, 749-750.

Sapolsky, R. M., Krey, L. C., McEwen, B. S., (1986). The neuroendocrinology of stress and aging: the glucocorticoid cascade hypothesis. Endocrine Reviews, 7, 284-301.

Sargent, P. A., Kjaer, K. H., Bench, C. J., Rabiner, E. A., Messa, C., Meyer, J., et al., (2000). Brain serotonin1A receptor binding measured by positron emission tomography with [11C]WAY-100635: effects of depression and antidepressant treatment. Archives of General Psychiatry, 57, 174-180.

Scrutton, H., Carbonnier, A., Cowen, P. J., Harmer, C., (2007). Effects of \{alpha\}-lactalbumin on emotional processing in healthy women. Journal of Psychopharmacology

Seckl, J. R., Fink, G., (1991). Use of in situ hybridization to investigate the regulation of hippocampal corticosteroid receptors by monoamines. The Journal of Steroid Biochemistry and Molecular Biology, 40, 685-688.

Segal, Z. V., Gemar, M., Williams, S., (1999). Differential cognitive response to a mood challenge following successful cognitive therapy or pharmacotherapy for unipolar depression. Journal of Abnormal Psychology, 108, 3-10.

Segal, Z. V., Kennedy, S., Gemar, M., Hood, K., Pedersen, R., Buis, T., (2006). Cognitive reactivity to sad mood provocation and the prediction of depressive relapse. Archives of General Psychiatry, 63, 749-755.

Selye, H., (1936). A syndrome produced by diverse nocuous agents. Nature, 138, 32.

Selye, H., (1976). Forty years of stress research: principal remaining problems and misconceptions. Canadian Medical Association Journal, 115, 53-56.

Serretti, A., Mandelli, L., Lorenzi, C., Pirovano, A., Olgiati, P., Colombo, C., et al., (2007). Serotonin transporter gene influences the time course of improvement of "core" de- 
pressive and somatic anxiety symptoms during treatment with SSRIs for recurrent mood disorders. Psychiatry Research, 149, 185-193.

Sheehan, D., Lecrubier, Y., Janavs, J., Knapp, E., Weiller, E., (1994). MINI International Neuropsychiatric Interview. Tampa, FL: University of South Florida.

Sheline, Y. I., Gado, M. H., Kraemer, H. C., (2003). Untreated depression and hippocampal volume loss. The American Journal of Psychiatry, 160, 1516-1518.

Smeets, T., Jelicic, M., Merckelbach, H., (2006). The effect of acute stress on memory depends on word valence. International Journal of Psychophysiology, 62, 30-37.

Smeraldi, E., Zanardi, R., Benedetti, F., Di Bella, D., Perez, J., Catalano, M., (1998). Polymorphism within the promoter of the serotonin transporter gene and antidepressant efficacy of fluvoxamine. Molecular Psychiatry, 3, 508-511.

Sobczak, S., Honig, A., Nicolson, N. A., Riedel, W. J., (2002a). Effects of acute tryptophan depletion on mood and cortisol release in first-degree relatives of type I and type II bipolar patients and healthy matched controls. Neuropsychopharmacology, 27, 834-842.

Sobczak, S., Riedel, W. J., Booij, I., Aan Het Rot, M., Deutz, N. E., Honig, A., (2002b). Cognition following acute tryptophan depletion: difference between first-degree relatives of bipolar disorder patients and matched healthy control volunteers. Psychological Medicine, 32, 503-515.

Stahl, S. M., (1998). Mechanism of action of serotonin selective reuptake inhibitors. Serotonin receptors and pathways mediate therapeutic effects and side effects. Journal of Affective Disorders, 51, 215-235.

Steckler, T., Holsboer, F., Reul, J. M., (1999). Glucocorticoids and depression. Baillieres Best Pract \& Research Clinical Endocrinology \& Metabolism, 13, 597-614.

Suhara, T., Takano, A., Sudo, Y., Ichimiya, T., Inoue, M., Yasuno, F., et al., (2003). High levels of serotonin transporter occupancy with low-dose clomipramine in comparative occupancy study with fluvoxamine using positron emission tomography. Archives of General Psychiatry, 60, 386-391.

Sullivan, P. F., Neale, M. C., Kendler, K. S., (2000). Genetic epidemiology of major depression: review and meta-analysis. The American Journal of Psychiatry, 157, 1552-1562.

Surtees, P. G., Wainwright, N. W., Willis-Owen, S. A., Luben, R., Day, N. E., Flint, J., (2006). Social adversity, the serotonin transporter (5-HTTLPR) polymorphism and major depressive disorder. Biological Psychiatry, 59, 224-229.

Tavares, J. V., Drevets, W. C., Sahakian, B. J., (2003). Cognition in mania and depression. Psychological Medicine, 33, 959-967.

Teasdale, J. D., (1988). Cognitive vulnerability to persistent depression. Cognition and Emotion, 2, 247-274.

Tull, M. T., Gratz, K. L., (2008). Further examination of the relationship between anxiety sensitivity and depression: the mediating role of experiential avoidance and difficulties engaging in goal-directed behavior when distressed. Journal of Anxiety Disorders, 22, 199-210.

Uher, R., McGuffin, P., (2008). The moderation by the serotonin transporter gene of environmental adversity in the aetiology of mental illness: review and methodological analysis. Molecular Psychiatry, 13, 131-146.

Ursin, H., Olff, M., (1993). Psychobiology of coping and defence strategies. Neuropsychobiology, 28, 66-71.

Van Breukelen, G. J., Van Dijk, K. R., (2007). Use of covariates in randomized controlled trials. Journal of the International Neuropsychological Society, 13, 903-904. 


\section{REFERENCES}

Van der Does, A. J., (2001). The effects of tryptophan depletion on mood and psychiatric symptoms. Journal of Affective Disorders, 64, 107-119.

Van der Does, W., (2002). Cognitive reactivity to sad mood: structure and validity of a new measure. Behaviour Research and Therapy, 40, 105-120.

van der Veen, F. M., Evers, E. A., Deutz, N. E., Schmitt, J. A., (2007). Effects of acute tryptophan depletion on mood and facial emotion perception related brain activation and performance in healthy women with and without a family history of depression. Neuropsychopharmacology, 32, 216-224.

Van Praag, H. M., (2004). Can stress cause depression? Progress in Neuropsychopharmacology and Biological Psychiatry, 28, 891-907.

Van Praag, H. M., de Kloet, E. R., Van Os, J., (2004). Stress, the brain and depression. Cambridge: Cambridge University Press.

Voineskos, A. N., Wilson, A. A., Boovariwala, A., Sagrati, S., Houle, S., Rusjan, P., et al., (2007). Serotonin transporter occupancy of high-dose selective serotonin reuptake inhibitors during major depressive disorder measured with [(11)C]DASB positron emission tomography. Psychopharmacology (Berl), 193, 539-545.

Von Bardeleben, U., Holsboer, F., (1989). Cortisol response to a combined dexamethasone-hCRH challenge in patients with depression. Journal of Neuroendocrinology, 1, 485-488.

Wald, F. D. M., Mellenbergh, G. J., (1990). De verkorte versie van de Nederlandse vertaling van de Profile of Mood States (POMS). Nederlands Tijdschrift voor de Psychologie, 45, 86-90.

Walderhaug, E., Magnusson, A., Neumeister, A., Lappalainen, J., Lunde, H., Refsum, H., et al., (2007). Interactive effects of sex and 5-HTTLPR on mood and impulsivity during tryptophan depletion in healthy people. Biological Psychiatry, 62, 593-599.

Watson, D., Clark, L. A., Tellegen, A., (1988). Development and validation of brief measures of positive and negative affect: The PANAS scales. Journal of Personality and Social Psychology, 54, 1063-1070.

Wendland, J. R., Martin, B. J., Kruse, M. R., Lesch, K. P., Murphy, D. L., (2006). Simultaneous genotyping of four functional loci of human SLC6A4, with a reappraisal of 5HTTLPR and rs25531. Molecular Psychiatry, 11, 224-226.

Wilhelm, K., Mitchell, P. B., Niven, H., Finch, A., Wedgwood, L., Scimone, A., et al., (2006). Life events, first depression onset and the serotonin transporter gene. The Bristish Journal of Psychiatry, 188, 210-215.

Williams, E., Stewart-Knox, B., Helander, A., McConville, C., Bradbury, I., Rowland, I., (2006). Associations between whole-blood serotonin and subjective mood in healthy male volunteers. Biological Psychology, 71, 171-174.

Williams, R. B., Marchuk, D. A., Gadde, K. M., Barefoot, J. C., Grichnik, K., Helms, M. J., et al., (2003). Serotonin-related gene polymorphisms and central nervous system serotonin function. Neuropsychopharmacology, 28, 533-541.

Williams, W. A., Shoaf, S. E., Hommer, D., Rawlings, R., Linnoila, M., (1999). Effects of acute tryptophan depletion on plasma and cerebrospinal fluid tryptophan and 5hydroxyindoleacetic acid in normal volunteers. Journal of Neurochemistry, 72, 16411647.

Willis-Owen, S. A., Turri, M. G., Munafo, M. R., Surtees, P. G., Wainwright, N. W., Brixey, R. D., et al., (2005). The serotonin transporter length polymorphism, neuroticism, and depression: a comprehensive assessment of association. Biological Psychiatry, 58, 451456. 
Wurtman, R. J., (2005). Genes, stress, and depression. Metabolism, 54, 16-19.

Young, S. N., Gauthier, S., (1981). Effect of tryptophan administration on tryptophan, 5hydroxyindoleacetic acid and indoleacetic acid in human lumbar and cisternal cerebrospinal fluid. Journal of Neurology, Neurosurgery \& Psychiatry, 44, 323-328.

Young, S. N., Smith, S. E., Pihl, R. O., Ervin, F. R., (1985). Tryptophan depletion causes a rapid lowering of mood in normal males. Psychopharmacology (Berl), 87, 173-177.

Yuwiler, A., Brammer, G. L., Morley, J. E., Raleigh, M. J., Flannery, J. W., Geller, E., (1981). Short-term and repetitive administration of oral tryptophan in normal men. Effects on blood tryptophan, serotonin, and kynurenine concentrations. Archives of General Psychiatry, 38, 619-626.

Zalsman, G., Huang, Y. Y., Oquendo, M. A., Burke, A. K., Hu, X. Z., Brent, D. A., et al., (2006). Association of a Triallelic Serotonin Transporter Gene Promoter Region (5HTTLPR) Polymorphism With Stressful Life Events and Severity of Depression. The American Journal of Psychiatry, 163, 1588-1593. 

DANKWOORD 
Graag wil ik een aantal mensen bedanken:

Allereerst wil ik mijn copromotor Dr. Rob Markus bedanken. Rob, heel veel dank voor de kans om te promoveren, het vertrouwen in mijn capaciteiten, voor de vrijheid in het onderzoek, voor je deskundigheid, en voor je optimisme.

Veel dank ook aan mijn promotor Prof. dr. Anita Jansen. Anita, bedankt dat je mijn promotor wilde zijn en voor jouw ondersteuning van mijn aanblijving.

Veel dank aan alle collega's van EP/CPS en EPU voor de gezellige en inspirerende werksfeer.

Bedanken möchte ich mich auch bei Prof. dr. Niels Schiller. Niels, danke, dass Du mir ermöglicht hast bereits als Student meine ersten Forschungserfahrungen zu sammeln und so meine Freude an Wissenschaft und Forschung zu entdecken.

Nicolette, Ramona en Gerda. Jullie waren geweldige kamergenootjes. Bedankt voor de mooie tijd!

Nicolette en Steffi: Ik ben heel blij dat jullie mijn paranimfen willen zijn!

Bedanken wil ik natuurlijk ook mijn studentassistenten Marjolein, Anja, Sarah, Jasper, Janneke, Matthis en Lauren voor hun grote hulp bij het verzamelen van de data.

Aan alle proefpersonen die deelgenomen hebben aan de experimenten: Bedankt voor jullie medewerking!

Liebe Freunde und Familie, von Herzen Dank für Freude, Hilfe, Leositting, interessante Gespräche, Müttercafes, Tratsch und Klatsch, Pommes und Champagner und vieles mehr im echten Leben!

Liebe Oma, liebe Christiane, von Herzen Dank für Eure Unterstützung! 
Liebe Mama, lieber Papa, ich kann Euch nicht genug danken für Eure Liebe und Unterstützung, alle Chancen und Möglichkeiten und Euer großes Vertrauen in mich. Liebe Edith, meine liebste Mama und Freundin: Danke für alles. Meine geliebten Jungs: Alex, Basti und Juli: Ihr seid die besten Brüder der Welt!

Georg und Leo, meine Männer, meine Familie, mein Glück: DANKE. 



\section{CURRICULUM VITAE}


Christine Firk was born on October 21, 1980 in Aachen, Germany. In June 2000, she graduated from secondary school (St. Leonhard Gymnasium, Aachen). In October 2000, she started studying English literature at RWTH Aachen University. In September 2001, she started studying Psychology at Maastricht University. She graduated in August 2005 and received her master's degree in Biological Psychology (cum laude). In October 2005, she started working as a PhD student at the Department of Clinical Psychological Science, Faculty of Psychology and Neuroscience, Maastricht University. Currently, she is a postdoctoral fellow at the Department of Neuropsychology and Psychopharmacology, Faculty of Psychology and Neuroscience, Maastricht University. Christine Firk is married to Georg Hilfrich. They have one son, Leonard (born 16 October 2007). 